

\title{
Torsion pour les variétés abéliennes de type I et II
}

\author{
Marc Hindry et Nicolas Ratazzi
}

Soit $A$ une variété abélienne définie sur un corps de nombres $K$. Le nombre de points de torsion définis sur une extension finie $L$ est borné polynomialement en terme du degré $[L: K]$ de $L$ sur $K$. Sous les trois hypothèses suivantes nous calculons l'exposant optimal dans cette borne, en terme de la dimension des sous-variétés abéliennes de $A$ et de leurs anneaux d'endomorphismes. Les trois hypothèses faites sur $A$ sont les suivantes : (1) $A$ est géométriquement isogène à un produit de variétés abéliennes simples de type I ou II dans la classification d'Albert ; (2) A est de "type Lefschetz" c'est-à-dire que le groupe de Mumford-Tate est le groupe des similitudes symplectiques commutant aux endomorphismes; (3) A vérifie la conjecture de Mumford-Tate. Le résultat est notamment inconditionnel (i.e., ces trois hypothèses sont vérifiées) pour un produit de variétés abéliennes simples de type I ou II et de dimension relative impaire. Par ailleurs nous prouvons, en étendant des résultats de Serre, Pink et Hall, la conjecture de Mumford-Tate pour quelques nouveaux cas de variétés abéliennes de type Lefschetz.

Let $A$ be an abelian variety defined over a number field $K$. The number of torsion points that are rational over a finite extension $L$ is bounded polynomially in terms of the degree $[L: K]$ of $L$ over $K$. Under the following three conditions we compute the optimal exponent for this bound, in terms of the dimension of abelian subvarieties and their endomorphism rings. The three hypotheses are the following: (1) $A$ is geometrically isogenous to a product of simple abelian varieties of type I or II, according to the Albert classification; (2) $A$ is of "Lefschetz type," that is, the Mumford-Tate group is the group of symplectic similitudes which commute with the endomorphism ring. (3) $A$ satisfies the Mumford-Tate conjecture. This result is unconditional (i.e., the three hypotheses are satisfied) for a product of simple abelian varieties of type I or II with odd relative dimension. Further, building on work of Serre, Pink and Hall, we also prove the Mumford-Tate conjecture for a few new cases of abelian varieties of Lefschetz type.

MSC2010 : primary 11G10; secondary 11F80, 14K15, 14KXX.

Mots-clefs : abelian varieties, Galois representations, Mumford-Tate group, torsion points, symplectic group. 


\section{Introduction}

Soit $A$ / $K$ une variété abélienne, définie sur un corps de nombres $K$, de dimension $g \geq 1$. Le classique théorème de Mordell-Weil assure que le groupe $A(F)$ des points $F$-rationnels de $A$ est de type fini pour toute extension finie $F / K$. Un problème naturel qui se pose est de déterminer le sous-groupe de torsion $A(F)_{\text {tors. }}$. Un premier problème consiste en fait à borner explicitement le cardinal de $A(F)_{\text {tors }}$ lorsque $A$ ou $F$ varient. Comme dans les articles [Ratazzi 2007 ; Hindry et Ratazzi 2012; 2010] auxquels ce papier fait suite, nous nous intéressons ici au cas où l'on fixe $A$ et où l'on fait varier $F$ parmi les extensions finies de $K$; l'objectif étant d'obtenir une borne avec une dépendance explicite et optimale en le degré $[F: K]$. Introduisons maintenant l'invariant que nous allons étudier.

Définition 1.1. On pose

$$
\gamma(A)=\inf \left\{x>0 \mid \forall F / K \text { finie, }\left|A(F)_{\text {tors }}\right| \ll[F: K]^{x}\right\} .
$$

La notation $\ll$ signifie qu'il existe une constante $C$, ne dépendant que de $A / K$, telle que l'on a $\left|A(F)_{\text {tors }}\right| \leq C[F: K]^{x}$.

On peut traduire la définition en le fait que $\gamma(A)$ est l'exposant le plus petit possible tel que pour tout $\varepsilon>0$, il existe une constante $C(\varepsilon)=C(A / K, \varepsilon)$ telle que pour toute extension finie $F / K$ on a

$$
\left|A(F)_{\mathrm{tors}}\right| \leq C(\varepsilon)[F: K]^{\gamma(A)+\varepsilon} .
$$

Un résultat général dû à Masser [1986] donne une borne simple :

$$
\gamma(A) \leq \operatorname{dim} A .
$$

Cette borne est optimale lorsque $A$ est une puissance d'une courbe elliptique avec multiplication complexe ; il est fort probable que la borne de Masser n'est jamais optimale dans les autres cas. L'invariant $\gamma(A)$ est calculé dans [Hindry et Ratazzi 2010] pour un produit de courbes elliptiques et, de manière différente, dans [Ratazzi 2007] pour une variété abélienne de type CM et dans [Hindry et Ratazzi 2012] pour une variété abélienne "générique". Le problème analogue pour les modules de Drinfeld est traité dans [Breuer 2010].

Nous notons $\operatorname{End}(A)$ l'anneau d'endomorphismes de $A / \bar{K}$ et posons $\operatorname{End}^{0}(A):=$ $\operatorname{End}(A) \otimes \mathbb{Q}$. On étudie dans ce texte les deux cas suivants :

- La variété abélienne $A$ est de type I, i.e., $E:=\operatorname{End}(A) \otimes \mathbb{Q}$ est un corps totalement réel de degré $e$; en particulier $g=h e$ où $h$ est entier.

— La variété abélienne $A$ est de type II, i.e., $D:=\operatorname{End}(A) \otimes \mathbb{Q}$ est une algèbre de quaternions totalement indéterminée de centre un corps totalement réel $E$ de degré $e$; en particulier $g=2 h e$ où $h$ est entier. 
Les deux autres cas (type III, i.e., algèbre de quaternions totalement définie, et type IV, i.e., algèbre à division sur un corps $\mathrm{CM}$ ) sont de nature différente et seront traités dans une autre publication.

Définition 1.2. L'entier $h$ précédent s'appelle la dimension relative de $A$.

On sait que, pour une telle variété abélienne de type I ou II, le groupe de Hodge est toujours contenu dans $\operatorname{Res}_{E / \mathbb{Q}} \mathrm{Sp}_{2 h}$ et est génériquement égal à ce dernier groupe. Lorsque $h$ est impair ou égal à 2, on sait que le groupe de Hodge est effectivement $\operatorname{Res}_{E / \mathbb{Q}} \mathrm{Sp}_{2 h}$ et, de plus, que la conjecture de Mumford-Tate est vraie (cf. le théorème 3.7 plus loin). Notons que le cas de type I avec $e=1$ correspond aux variétés abéliennes de type GSp (dans la terminologie de [Hindry et Ratazzi 2012]).

Pour tout premier $\ell$, on note $T_{\ell}(A)=\lim _{\longleftarrow} A\left[\ell^{n}\right]$ le module de Tate $\ell$-adique de $A$; considérant l'action du groupe de Galois $G_{K}:=\mathrm{Gal}(\bar{K} / K)$ sur les points de $\ell^{\infty}$-torsion, on associe naturellement à $A / K$, la représentation $\ell$-adique

$$
\rho_{\ell, A}: G_{K} \rightarrow \mathrm{GL}\left(T_{\ell}(A)\right) \simeq \mathrm{GL}_{2 g}\left(\mathbb{Z}_{\ell}\right) .
$$

On pose $V_{\ell}(A)=T_{\ell}(A) \otimes_{\mathbb{Z}_{\ell}} \mathbb{Q}_{\ell}$. Rappelons que $V_{\ell}(A)$ est naturellement le dual de $H_{\text {ét }}^{1}\left(A \times \bar{K}, \mathbb{Q}_{\ell}\right)$ et que, si l'on note $V=V(A)=H_{1}(A, \mathbb{Q})$, alors, comme $\mathbb{Q}$-espace vectoriel, $V \cong \mathbb{Q}^{2 g}$, et $V \otimes_{\mathbb{Q}} \mathbb{Q}_{\ell}$ s'identifie naturellement à $V_{\ell}(A)$. Si $\psi: V \times V \rightarrow \mathbb{Q}$ est la forme symplectique associée à une polarisation, sa tensorisée par $\mathbb{Q}_{\ell}$ s'identifie avec la forme symplectique provenant de l'accouplement de Weil associé à la même polarisation $T_{\ell}(A) \times T_{\ell}(A) \rightarrow \mathbb{Z}_{\ell}(1) \cong \mathbb{Z}_{\ell}$.

Définition 1.3. Le groupe de Lefschetz de $A$, noté $L(A)$, est le commutateur ${ }^{1}$, en tant que $\mathbb{Q}$-groupe algébrique, de $\operatorname{End}^{0}(A)$ dans $\operatorname{Sp}(V, \psi)=\operatorname{Sp}_{2 g}$.

On note également $G_{\ell}$ ou $G_{\ell, A}$ si besoin, l'enveloppe algébrique de l'image de $\rho_{\ell \infty}$, et $H_{\ell, A}$ la composante neutre de $G_{\ell} \cap \operatorname{SL}\left(V_{\ell}\right)$, c'est-à-dire

$$
G_{\ell, A}:=\left(\overline{\rho_{\ell, A}\left(G_{K}\right)^{\mathrm{Zar}}}\right)^{0} \quad \text { et } \quad H_{\ell, A}:=\left(G_{\ell} \cap \operatorname{SL}\left(V_{\ell}\right)\right)^{0} .
$$

On note $\operatorname{MT}(A)$ le groupe de Mumford-Tate et $\operatorname{Hdg}(A)$ le groupe de Hodge d'une variété abélienne $A$ (cf. par exemple [Pink 1998; Moonen 1999]). La relation entre les deux groupes est $\operatorname{MT}(A)=\mathbb{G}_{m} \cdot \operatorname{Hdg}(A)$ et $\operatorname{Hdg}(A)$ est la composante neutre de $\operatorname{MT}(A) \cap \operatorname{SL}(V)$.

Le groupe des homothéties est contenu dans $G_{\ell, A}$; une autre manière de définir le $\mathbb{Q}_{\ell}$-groupe algébrique $H_{\ell, A}$, pour se ramener dans $\operatorname{Hdg}(A)$, est de le voir comme l'enveloppe algébrique de $\rho\left(\operatorname{Gal}\left(\bar{K} / K\left(\mu_{\ell^{\infty}}\right)\right)\right.$, c'est-à-dire la composante neutre de

1. Ce groupe algébrique est connexe, sauf pour les variétés de type III, cf. Murty [1984] et Milne [1999]. 
la clôture de Zariski de l'image de Galois dans $\mathrm{GL}_{2 g, \mathbb{Q}_{\ell}}$. On sait alors que l'on a toujours

$$
H_{\ell, A} \subset \operatorname{Hdg}(A)_{\mathbb{Q}_{\ell}} \subset L(A)_{\mathbb{Q}_{\ell}} .
$$

Remarquons que ces deux inclusions n'ont pas le même statut : la conjecture de Mumford-Tate prédit que la première inclusion est toujours une égalité, alors que la seconde inclusion peut ne pas être une égalité.

Définition 1.4. Nous dirons que $A$ est de type Lefschetz si $\operatorname{Hdg}(A)=L(A)$ et pleinement de type Lefschetz si pour tout premier $\ell$, on a $H_{\ell, A}=L(A)_{\mathbb{Q}_{\ell}}$.

Remarque 1.5. Dans la définition précédente, il suffit en fait de supposer que $H_{\ell, A}=L(A)_{\mathbb{Q}_{\ell}}$ pour un premier $\ell$ (cf. [Larsen et Pink 1995, Theorem 4.3]).

Ainsi une variété abélienne de type Lefschetz est pleinement de type Lefschetz si elle vérifie la conjecture de Mumford-Tate.

Théorème 1.6. Soit A une variété abélienne géométriquement simple de type I ou II définie sur un corps de nombres, dont le centre de l'algèbre d'endomorphismes est un corps de nombres totalement réel $E$ de degré $e=[E: \mathbb{Q}]$. Posons $d:=$ $\sqrt{\left[\operatorname{End}^{0}(A): E\right]}$ (i.e., $d=1$ ou 2). On suppose que A est pleinement de type Lefschetz; on a alors

$$
\gamma(A)=\frac{2 d h e}{1+2 e h^{2}+h e}=\frac{2 \operatorname{dim} A}{\operatorname{dim} \operatorname{MT}(A)} .
$$

Remarque 1.7. Il est intéressant d'observer que l'exposant $\gamma(A)$ est beaucoup plus petit que la borne $\gamma(A) \leq g$ donnée par Masser; par exemple, pour toute variété abélienne vérifiant les hypothèses du théorème, on a pour le type I (resp. le type II) $\gamma(A)<2 / 3($ resp. $\gamma(A)<4 / 3)$.

Pour énoncer la deuxième partie du corollaire suivant, nous introduisons la notation

$$
\Sigma=\left\{g \geq 1 \mid \exists k \geq 3 \text {, impair, } \exists a \geq 1,2 g=(2 a)^{k} \text { ou } 2 g=\left(\begin{array}{c}
2 k \\
k
\end{array}\right)\right\} .
$$

Pour énoncer la troisième partie du corollaire suivant, rappelons que si une variété abélienne $A$ a réduction semi-stable en une place $v$, la composante neutre de la fibre spéciale est une extension d'une variété abélienne par un tore; la dimension de ce tore s'appelle la dimension torique. Remarquons également (cf. la preuve du théorème 10.7) que si la variété abélienne $A$ est de type I (resp. de type II) et possède mauvaise réduction semi-stable en une place, alors la dimension torique de la fibre spéciale est un multiple de $e$ (resp. de $2 e$ ). En rassemblant les cas où l'on sait démontrer qu'une variété abélienne de type I ou II est pleinement de type Lefschetz (voir théorème 3.7 et section 10) on obtient le corollaire suivant. 
Corollaire 1.8. Soit $E$ un corps de nombres totalement réel de degré e $=[E: \mathbb{Q}]$. Soit A une variété abélienne définie sur un corps de nombres, telle que A est géométriquement simple de type I ou II et le centre de son algèbre d'endomorphismes est E. On suppose de plus que l'une quelconque des trois hypothèses suivantes est satisfaite:

(1) La dimension relative hest un nombre impair ou égal à 2.

(2) On a $e=1$ (ou encore $E=\mathbb{Q}$ ) et la dimension relative $h$ n'appartient pas au sous-ensemble exceptionnel $\Sigma$.

(3) La variété abélienne A est de type I (resp. II) et possède une place de mauvaise réduction semi-stable avec dimension torique e (resp. avec dimension torique $2 e$ ).

On a alors

$$
\gamma(A)=\frac{2 d h e}{1+2 e h^{2}+h e}=\frac{2 \operatorname{dim} A}{\operatorname{dim} \mathrm{MT}(A)} .
$$

Remarque 1.9. Lorsque $e=1$ et $A$ de type I, le point (2) correspond au cas "générique" traité dans [Hindry et Ratazzi 2012].

Remarque 1.10. Comme cela est démontré par Noot [1995], pour chaque type de groupe de Hodge, il existe des variétés abéliennes définies sur un corps de nombres, ayant ce groupe de Hodge donné et vérifiant la conjecture de Mumford-Tate. En particulier, pour tout corps de nombres totalement réel $E$ de degré $e$ sur $\mathbb{Q}$ et tout entier $h$, il existe des variétés abéliennes $A$ définies sur un corps de nombres, de dimension $e h$ (resp. 2eh) telles que $\operatorname{End}^{0}(A)=E$ (resp. $\operatorname{End}^{0}(A)$ est une algèbre de quaternions indéfinie sur $E$ ) et qui sont pleinement de Lefschetz. Ces variétés abéliennes vérifient donc les hypothèses du théorème.

Remarque 1.11. La preuve fournit une inégalité légèrement plus précise que $\left|A(L)_{\text {tor }}\right| \ll_{\varepsilon}[L: K]^{\gamma(A)+\varepsilon}$ de la forme

$$
\left|A(L)_{\text {tor }}\right| \ll[L: K]^{\gamma(A)+c_{1} / \log \log [L: K]} .
$$

On peut récrire ce résultat en terme de minoration du degré de l'extension engendrée par un sous-groupe de torsion; nous donnons la teneur du résultat ci-dessous dans le cas de l'extension engendrée par un seul point de torsion.

Théorème 1.12. Soit $A / K$ une variété abélienne géométriquement simple de type I ou II, de dimension relative h et pleinement de type Lefschetz. Il existe une constante $c_{1}:=c_{1}(A, K)>0$ telle que pour tout point de torsion $P$ d'ordre $n$ dans $A(\bar{K})$, on a la minoration

$$
[K(P): K] \geq c_{1}^{\omega(n)} n^{2 h} .
$$


Remarque 1.13. On peut affaiblir légèrement l'énoncé en écrivant l'inégalité sous la forme

$$
[K(P): K] \gg n^{2 h-c / \log \log n} \quad \text { ou encore } \quad[K(P): K] \gg \gg_{\varepsilon} n^{2 h-\varepsilon} \text { pour tout } \varepsilon>0 .
$$

Par ailleurs notons que, toujours pour un point $P$ d'ordre $n$, on a trivialement $[K(P): K] \leq n^{2 g}$. On voit donc que dans le cas totalement générique (appelé "type GSp" dans [Hindry et Ratazzi 2012]) tous les points d'ordre $n$ engendrent des corps de degrés comparables $n^{2 g} \geq[K(P): K] \gg n^{2 g-\varepsilon}$, mais qu'il n'en est plus de même quand $e \geq 2$.

Nous étendons les résultats au cas d'une variété abélienne géométriquement isogène à un produit de variétés abéliennes de type I ou II.

Théorème 1.14. Soit $A / K$ une variété abélienne isogène sur $\bar{K}$ à un produit $A_{1}^{n_{1}} \times \cdots \times A_{d}^{n_{d}}$ avec $A_{i}$ non $\bar{K}$-isogène à $A_{j}$ pour $i \neq j$. On suppose que chaque facteur $A_{i}$ est de type I ou II et est pleinement de type Lefschetz. Pour tout sousensemble non vide $I$ de $[1, d]$ on note $A_{I}:=\prod_{i \in I} A_{i}$. On note $e_{i}$ la dimension $d u$ centre de $\operatorname{End}^{0}\left(A_{i}\right)$ et $h_{i}$ la dimension relative de $A_{i}$. Enfin on pose $d_{i}=1$ (resp. $\left.d_{i}=2\right)$ si $A_{i}$ est de type I (resp. de type II). On a alors

$$
\gamma(A)=\max _{I} \frac{2 \sum_{i \in I} n_{i} d_{i} h_{i} e_{i}}{1+\sum_{i \in I} 2 e_{i} h_{i}^{2}+h_{i} e_{i}}=\max _{I} \frac{2 \sum_{i \in I} n_{i} \operatorname{dim} A_{i}}{\operatorname{dim} \operatorname{MT}\left(A_{I}\right)} .
$$

Corollaire 1.15. Soit A une variété abélienne géométriquement isogène à un produit $A_{1}^{n_{1}} \times \cdots \times A_{d}^{n_{d}}$ avec $A_{i}$ non $\bar{K}$-isogène à $A_{j}$ pour $i \neq j$. On suppose que chaque facteur $A_{i}$ est de type I ou II et vérifie l'une quelconque des trois hypothèses suivantes:

(1) Le nombre $h_{i}$ est impair ou égal à 2.

(2) On a $e_{i}=1$ (i.e., $E_{i}=\mathbb{Q}$ ) et $h_{i} \notin \Sigma$.

(3) La variété abélienne $A_{i}$ est de type I (resp. II) et possède une place de mauvaise réduction semi-stable avec dimension torique $e_{i}$ (resp. avec dimension torique $\left.2 e_{i}\right)$.

On a alors, avec les notations du théorème 1.14,

$$
\gamma(A)=\max _{I} \frac{2 \sum_{i \in I} n_{i} d_{i} h_{i} e_{i}}{1+\sum_{i \in I} 2 e_{i} h_{i}^{2}+h_{i} e_{i}}=\max _{I} \frac{2 \sum_{i \in I} n_{i} \operatorname{dim} A_{i}}{\operatorname{dim} \operatorname{MT}\left(A_{I}\right)} .
$$

Remarque 1.16. Dans le contexte du corollaire 1.15, l'analogue du théorème 1.12 dit simplement que

$$
[K(P): K] \geq c_{1}^{\omega(n)} n^{2 h_{0}},
$$

où $h_{0}$ est le minimum des $h_{i}$. 
Réductions. Le problème que nous étudions est clairement invariant par deux modifications : remplacer $K$ par une extension finie $K^{\prime}$ et remplacer $A$ par une variété abélienne $A^{\prime}$ isogène à $A$. Quitte à effectuer une extension finie de $K$ et à remplacer $A$ par une variété isogène, nous pouvons donc supposer, et nous supposerons dans le reste de l'article, les propriétés suivantes vérifiées par $A / K$ :

(1) L'anneau des endomorphismes définis sur $K$ est égal à l'anneau des endomorphismes définis sur $\bar{K}$; on le notera donc $\operatorname{End}(A)$.

(2) L'anneau des endomorphismes $\operatorname{End}(A)$ est un ordre maximal dans $\operatorname{End}(A) \otimes \mathbb{Q}$.

(3) La variété abélienne s'écrit $A=A_{1}^{n_{1}} \times \cdots \times A_{r}^{n_{r}}$, avec $1 \leq r, 1 \leq n_{i}$ et des variétés abéliennes $A_{i}$ absolument simples non isogènes deux à deux.

(4) L'adhérence de Zariski de $\rho_{\ell^{\infty}, A}\left(G_{K}\right)$ est connexe.

(5) Les représentations $\rho_{\ell^{\infty}, A}$ sont indépendantes sur $K$.

En effet la possibilité de l'obtention des trois premières propriétés par extension de $K$ et isogénie découle des propriétés générales des variétés abéliennes, tandis que les points (4) et (5) s'obtiennent par une extension adéquate du corps $K$, en invoquant deux résultats subtils de Serre [1986a; 2013]. Le point (5) est rappelé, ainsi que la définition de "représentations indépendantes" à la proposition 3.2.

Remarquons qu'on pourrait également songer à imposer que $A$ soit principalement polarisée, mais cela forcerait à renoncer à la propriété (2), il nous a semblé plus commode de préserver cette dernière.

Plan. Le plan de ce texte est le suivant : Dans la section suivante on rassemble un certain nombre de lemmes de théorie des groupes et de combinatoire. Dans les sections 3 et 4 on décrit les accouplements $\lambda$-adiques déduits de celui de Weil, ainsi que l'étude des propriétés des représentations galoisiennes qui sont utilisées dans la suite. La section 5 étudie la partie cyclotomique de ces représentations. Les sections 6 et 7 contiennent les preuves des deux théorèmes cités en introduction (théorèmes 1.6 et 1.14), d'abord dans le cas d'une variété abélienne simple et pour un groupe annulé par $\ell$ puis dans le cas général. Les preuves sont en fait écrites pour $\ell$ assez grand et on indique dans la huitième section comment on peut modifier les preuves pour traiter les "petits" nombres premiers de manière similaire. La courte neuvième section contient la démonstration de la minoration du degré de l'extension engendrée par un point de torsion (théorème 1.12). La dixième et dernière section est un appendice indépendant du reste de l'article (sauf pour les notations), dans lequel nous montrons comment déduire un énoncé du type "conjecture forte de Mumford-Tate" de l'énoncé usuel de la conjecture de Mumford-Tate et expliquons comment prouver les quelques cas supplémentaires de la conjecture de Mumford-Tate ne figurant pas dans la littérature et énoncés dans les corollaires 1.8 et 1.15 . 
Notations. Dans tout le reste de cet article, nous utiliserons les notations suivantes :

$$
[L: K], \quad(G: H), \quad[G, G]
$$

pour désigner respectivement, le degré de l'extension de corps $L$ sur $K$, l'indice du sous-groupe $H$ de $G$ dans $G$, le groupe des commutateurs de $G$.

Par ailleurs nous utiliserons les deux notations supplémentaires suivantes : l'égalité à indice fini près $\asymp$ et la presque égalité $\stackrel{\circ}{=}$ Commençons par définir l'égalité à indice fini près : $\mathrm{Si} L_{1}, L_{2}$ sont des corps de nombres contenus dans un corps $L$ (en pratique nos corps seront tous contenus dans $K\left(A_{\text {tor }}\right)$ ou, si l'on préfère dans $\overline{\mathbb{Q}}$ ou même $\mathbb{C}$ ) qui dépendent de $A / K$ et d'un autre ensemble de paramètres $\Lambda$, nous écrirons $L_{1} \asymp L_{2}$ pour dire qu'il existe une constante $\mathrm{C}(\mathrm{A} / \mathrm{K})$ ne dépendant que de $A / K$ telle que les inégalités

$$
\left[L_{1}: L_{1} \cap L_{2}\right] \leq C(A / K) \quad \text { et } \quad\left[L_{2}: L_{1} \cap L_{2}\right] \leq C(A / K)
$$

sont vraies pour toutes valeurs des paramètres dans l'ensemble $\Lambda$. De même si $G_{1}$ et $G_{2}$ sont des sous-groupes d'un même groupe et dépendant d'un ensemble de paramètres $\Lambda$, nous écrirons $G_{1} \asymp G_{2}$ pour dire qu'il existe une constante $\mathrm{C}(\mathrm{A} / \mathrm{K})$ ne dépendant que de $A / K$ telle que les inégalités $\left(G_{1}: G_{1} \cap G_{2}\right) \leq C(A / K)$ et $\left(G_{2}: G_{1} \cap G_{2}\right) \leq C(A / K)$ sont vraies pour toutes valeurs des paramètres dans l'ensemble $\Lambda$. Nous utiliserons la même notation pour des nombres. Ainsi, si $N_{1}, N_{2}$ sont deux nombres (par exemple des cardinaux de groupes ou des degrés d'extensions) $N_{1} \asymp N_{2}$ signifie qu'il existe deux constantes $C_{1}$ et $C_{2}$, indépendantes des paramètres, telles que $C_{1} N_{1} \leq N_{2} \leq C_{2} N_{1}$ (voir, par exemple, le lemme 6.4 pour une utilisation de cette dernière notation).

Concernant la presque égalité, dire que $E \stackrel{\circ}{=} F$ signifie par définition que $E \asymp F$ et que de plus il y a en fait égalité $E=F$ pour toutes les valeurs des paramètres dans $\Lambda$ sauf éventuellement un nombre fini (dépendant éventuellement de $A / K$ ).

Un exemple typique d'utilisation de la notation précédente consiste par exemple (cf. proposition 5.2) à écrire, pour $A / K$ une variété abélienne simple de type I ou II de dimension relative $h$ et pleinement de type Lefschetz, que l'on a

$$
\left[\rho_{\ell}\left(G_{K}\right), \rho_{\ell}\left(G_{K}\right)\right] \stackrel{\circ}{=} \operatorname{Sp}_{2 h}\left(\mathcal{O}_{E} / \ell \mathcal{O}_{E}\right),
$$

ceci signifiant qu'il existe une constante $\ell_{0}(A / K)$ dépendant de $A / K$ telle qu'il a égalité pour tout $\ell \geq \ell_{0}(A / K)$ et que de plus pour tout $\ell$ les deux groupes sont commensurables, i.e., l'indice de l'intersection des deux groupes dans l'un et l'autre est fini.

Enfin, si $L_{1}, L_{2}$ sont des corps de nombres qui dépendent de $A / K$ et d'un autre ensemble de paramètres $\Lambda$, nous écrirons $\left[L_{1}: \mathbb{Q}\right] \ll\left[L_{2}: \mathbb{Q}\right]$ pour dire qu'il existe une constante $\mathrm{C}(\mathrm{A} / \mathrm{K})$ ne dépendant que de $A / K$ telle que l'inégalité $\left[L_{1}: \mathbb{Q}\right] \leq C(A / K)\left[L_{2}: \mathbb{Q}\right]$ est vraie uniformément sur $\Lambda$. 


\section{Lemmes de groupes}

Soit $E / \mathbb{Q}$ un corps de nombres, d'anneau d'entiers $\mathcal{O}_{E}$. Si $\ell$ est un premier et $\lambda$ une place de $\mathcal{O}_{E}$ au dessus de $\ell$, on note $\mathcal{O}_{\lambda}$ le complété de $\mathcal{O}_{E}$ selon $\lambda$. De même on note $\mathbb{F}_{\lambda}$ le corps résiduel correspondant.

Nous rappelons maintenant des objets et notations provenant de [Hindry et Ratazzi 2012] que nous utiliserons ensuite. Soit $V$ un $\mathbb{Q}$-espace vectoriel muni d'une forme symplectique (dans la suite du papier nous utiliserons ceci avec $V=H_{1}(A(\mathbb{C}), \mathbb{Q})$ ). Dans les lemmes suivants nous introduisons $\left(e_{1}, \ldots, e_{2 g}\right)$ une base symplectique de $V$. (i.e., pour tout $1 \leq i, j \leq g, e_{i} \cdot e_{g+i}=+1$ et $e_{i} \cdot e_{j}=0$ si $|i-j| \neq 0$ ).

Lemme 2.1. Soit $0 \leq s \leq r \leq g$ avec $r \geq 1$. Définissons $P_{r, s}$ le sous-groupe algébrique de $\mathrm{Sp}_{2 g}$ fixant les vecteurs $e_{1}, \ldots, e_{r}$ et les vecteurs $e_{g+1}, \ldots, e_{g+s}$, c'est-à-dire

$$
P_{r, s}:=\left\{M \in \mathrm{Sp}_{2 g} \mid M e_{i}=e_{i}, i \in[1, r] \cup[g+1, g+s]\right\} .
$$

Alors, $P_{r, s}$ est lisse sur $\mathcal{O}_{E}$ et sa codimension dans $\mathrm{Sp}_{2 g}$ est $:$

$$
\operatorname{codim} P_{r, s}=2 s g+2 r g-r s-\frac{r(r-1)}{2}-\frac{s(s-1)}{2} .
$$

Démonstration. Il s'agit du lemme 2.24 de [Hindry et Ratazzi 2012] (énoncé sur $\mathbb{Z}$ mais valable par la même preuve sur $\mathcal{O}_{E}$ ).

Lemme 2.2. Soit $\lambda$ une place de $\mathcal{O}_{E}$ au dessus d'un premier $\ell$ de $\mathbb{Z}$. En introduisant les groupes

$$
\begin{aligned}
D_{0} & :=\left\{\left(\begin{array}{cc}
I & 0 \\
0 & \alpha I
\end{array}\right) \in \mathrm{GL}_{2 g}\left(\mathcal{O}_{\lambda}\right) \mid \alpha \in \mathbb{Z}_{\ell}^{\times}\right\}, \\
G_{\lambda} & :=\left\{M \in \operatorname{GSp}_{2 g}\left(\mathcal{O}_{\lambda}\right) \mid \operatorname{mult}(M) \in \mathbb{Z}_{\ell}^{\times}\right\},
\end{aligned}
$$

on $a$

$$
D_{0} \cdot \operatorname{Sp}_{2 g}\left(\mathcal{O}_{\lambda}\right)=G_{\lambda}
$$

Le même énoncé vaut en remplaçant $\mathcal{O}_{\lambda}$ par $\mathbb{F}_{\lambda}$ et $\mathbb{Z}_{\ell}$ par $\mathbb{F}_{\ell}$.

Démonstration. Il s'agit du lemme 2.12 de [Hindry et Ratazzi 2012] dans sa version $\lambda$-adique : soit $M \in G_{\lambda}$ de multiplicateur $\operatorname{mult}(M)$. La matrice

$$
\left(\begin{array}{cc}
I_{g} & 0 \\
0 & \operatorname{mult}(M) I_{g}
\end{array}\right) M
$$

est dans $\operatorname{Sp}_{2 g}\left(\mathcal{O}_{\lambda}\right)$.

Lemme 2.3. Soit $G_{E}$ un sous-groupe algébrique sur $E$ de $\mathrm{GL}_{E}$. Soit $t \in \mathbb{N}^{*}$ et soit $\mathcal{G}_{1, E}, \ldots, \mathcal{G}_{t, E}$ une suite de sous-groupes algébriques de $G_{E}$. On note $G$ (respectivement $\mathcal{G}_{i}$ ) l'adhérence de Zariski de $G_{E}$ (respectivement de $\mathcal{G}_{i, E}$ ) dans $\mathrm{GL}_{\mathcal{O}_{E}}$ sur $\mathcal{O}_{E}$. Il existe des constantes $C_{1}>0, C_{2}>0$ telles que la propriété 
suivante est vraie: soient $\ell$ un premier de $\mathbb{Z}$ et $\lambda$ une place de $\mathcal{O}_{E}$ au-dessus de $\ell$. Soient $G_{1} \subset G_{2} \subset \cdots \subset G_{t}$ une suite de sous-groupes algébriques sur $\mathcal{O}_{\lambda}$ de $G_{\mathcal{O}_{\lambda}}$. On suppose que pour tout $i$, le groupe $G_{i}$ est conjugué sur $\mathbb{F}_{\lambda}$ à $\mathcal{G}_{i}$. On note $g_{i}:=\operatorname{dim} \mathcal{G}_{i}=\operatorname{dim} G_{i}$ et $d_{i}:=\operatorname{codim}_{G} \mathcal{G}_{i}=\operatorname{codim}_{G_{\mathcal{O}_{\lambda}}} G_{i}$ et on pose, pour toute suite croissante d'entiers $0=m_{0}<m_{1}<m_{2}<\cdots<m_{t}$ :

$$
H\left(m_{1}, \ldots, m_{t}\right)=\left\{M \in G\left(\mathcal{O}_{\lambda}\right) \mid M \in G_{i} \quad \bmod \lambda^{m_{i}}\right\} .
$$

Pour tous les $\ell$ tels que $G$ et les $\mathcal{G}_{i}$ sont lisses sur $\mathbb{F}_{\lambda}$, on a alors

$$
\begin{aligned}
C_{1} \times\left(G\left(\mathcal{O}_{\lambda}\right): H\left(m_{1}, \ldots, m_{t}\right)\right) & \geq \operatorname{Card}\left(\mathbb{F}_{\lambda}\right)^{\sum_{i=1}^{t} d_{i}\left(m_{i}-m_{i-1}\right)} \\
& \geq C_{2} \times\left(G\left(\mathcal{O}_{\lambda}\right): H\left(m_{1}, \ldots, m_{t}\right)\right) .
\end{aligned}
$$

Démonstration. Il s'agit du lemme 2.4 de [Hindry et Ratazzi 2012] dont la preuve reste valable, en remplaçant $\mathbb{Z}$ par $\mathcal{O}_{E}$, ainsi que $\ell$ par $\lambda$ et $\mathbb{F}_{\ell}$ par $\mathbb{F}_{\lambda}$.

Nous donnons dans ce qui suit l'analogue d'un lemme prouvé pour $\mathrm{SL}_{2}\left(\mathbb{Z}_{\ell}\right)$ par Serre. L'énoncé en vue est le suivant, où les notations suivantes sont utilisées.

Définition 2.4. Nous dirons qu'une sous-algèbre de Lie de $g l_{m}$ possède la propriété $(\mathcal{C N})$ (des carrés nuls) si elle est engendrée, comme espace vectoriel, par des matrices de carré nul.

Exemple 2.5. Les algèbres $\mathfrak{s l}_{m}$ et $\mathfrak{s p}_{2 m}$ ont la propriété $(\mathcal{C N})$ mais pas $\mathfrak{s o}_{m}$. Les matrices $E_{i j}$ ayant un seul coefficient non nul (sur la $i$-ème ligne et $j$-ème colonne) sont de carré nul. En dimension 2, on a de même que $\left(\begin{array}{cc}a & 1 \\ -a^{2} & -a\end{array}\right)$ est de carré nul, donc

$$
\left(\begin{array}{cc}
a & b \\
c & -a
\end{array}\right)=\left(\begin{array}{cc}
a & 1 \\
-a^{2} & -a
\end{array}\right)+\left(\begin{array}{cc}
0 & b-1 \\
0 & 0
\end{array}\right)+\left(\begin{array}{cc}
0 & 0 \\
c+a^{2} & 0
\end{array}\right)
$$

est bien somme de matrices de carré nul. Quand $m$ est quelconque, on en tire aisément que les matrices diagonales de trace nulle sont sommes de matrices de carré nul. L'algèbre $\mathfrak{s p}_{2 m}$ est l'algèbre des matrices $\left(\begin{array}{cc}A & B \\ C & D\end{array}\right)$ telles que $D+{ }^{t} A=0$, $B$ et $C$ sont symétriques. On écrit aisément une telle matrice comme somme de matrices :

$$
\left(\begin{array}{ll}
0 & S \\
0 & 0
\end{array}\right), \quad\left(\begin{array}{ll}
0 & 0 \\
S & 0
\end{array}\right), \quad\left(\begin{array}{cc}
\delta & \delta \\
-\delta & -\delta
\end{array}\right), \quad\left(\begin{array}{cc}
U & 0 \\
0 & -{ }^{t} U
\end{array}\right),
$$

où $S$ est symétrique, $U$ a trace nulle et $\delta$ est la matrice dont le seul terme non nul est dans le coin supérieur gauche et vaut 1 ; les trois premières sont de carré nul et la quatrième est somme de matrices de carrés nuls d'après la propriété pour $\mathfrak{s l}_{m}$. Enfin notons qu'une matrice anti-symétrique de carré nul est elle-même nulle, donc $\mathfrak{s o}_{n}$ ne possède pas la propriété des carrés nuls.

Notations. On note $\mathcal{O}$ l'anneau d'entiers d'un corps $p$-adique, $\varpi$ une uniformisante, $\mathbb{F}=\mathcal{O} / \varpi \mathcal{O}$ le corps résiduel et $e$ l'indice de ramification, i.e., $p=\varpi^{e} u$ avec $u \in \mathcal{O}^{\times}$. 
Lemme 2.6. Soit $G$ un sous-groupe algébrique lisse de $\mathrm{GL}_{m} / \mathcal{O}$ et $H$ un sousgroupe fermé de $G(\mathcal{O})$. Considérons, pour tout entier $n \geq 1$, les applications $\pi_{n}: H \rightarrow \mathrm{GL}_{m}\left(\mathcal{O} / \varpi^{n} \mathcal{O}\right)$. Alors on $a:$

(1) Si $\pi_{e+1}(H)=G\left(\mathcal{O} / \varpi^{e+1} \mathcal{O}\right)$ et $p \geq e+2$, alors $H=G(\mathcal{O})$; si $p \leq e+1$ et $\pi_{m}$ surjective avec $m \geq(e p+1) /(p-1)$, alors $H=G(\mathcal{O})$.

(2) Si $p \geq 2 e+3, \pi_{e}(H)=G(\mathcal{O} / \varpi)$ et Lie $\left(G_{\mathbb{F}}\right)$ a la propriété $(\mathcal{C N})$ alors $H=G(\mathcal{O})$.

En particulier, lorsque $G=\mathrm{SL}_{m}$ ou $\mathrm{Sp}_{m}$, si $p \geq 5$ et $K / \mathbb{Q}_{p}$ non ramifié, alors $\pi_{1}(H)=G(\mathbb{F})$ entraîne $H=G(\mathcal{O})$.

Démonstration. Notons $\mathcal{L}:=\operatorname{Lie}\left(G_{\mathbb{E}}\right)$. Commençons par observer que, d'après l'hypothèse de lissité, si une matrice s'écrit $M=I+\varpi^{n} B$ alors $\pi_{n+1}(M) \in$ $G\left(\mathcal{O} / \varpi^{n+1} \mathcal{O}\right)$ si et seulement si $\pi_{1}(B) \in \mathcal{L}$. On prouve maintenant par récurrence sur $n$ que la projection $H \rightarrow G\left(\mathcal{O} / \varpi^{n} \mathcal{O}\right)$ est surjective et donc que $H$ est dense dans $G(\mathcal{O})$ et donc égal à ce dernier. Supposons la propriété vraie au cran $n$ et montrons-la au cran $n+1$. Soit donc $A \in G(\mathcal{O})$, on sait donc qu'il existe $A_{1} \in H$ telle que $A \equiv A_{1}\left[\varpi^{n}\right]$ et, quitte à remplacer $A$ par $A A_{1}^{-1}$ on peut supposer que $A \equiv I\left[\varpi^{n}\right]$ soit encore $A=I+\varpi^{n} B$ avec donc $\pi_{1}(B) \in \mathcal{L}$. Par hypothèse, il existe $Z \in H$ telle que $Z \equiv I+\varpi^{n-e} B\left[\varpi^{n-e+1}\right]$ ou encore $Z=I+\varpi^{n-e} B+\varpi^{n-e+1} C$. Posons $Y:=Z^{p}$ alors

$Y=I+p \varpi^{n-e}(B+\varpi C)+\sum_{h=2}^{p-1}\left(\begin{array}{l}p \\ h\end{array}\right) \varpi^{h(n-e)}(B+\varpi C)^{h}+\varpi^{p(n-e)}(B+\varpi C)^{p}$.

On a $e+h(n-e) \geq n+1$ si $n \geq e+1$ et $p(n-e) \geq n+1$ si $n \geq(e p+1) /(p-1)$; ainsi si $p \geq e+2$ on voit que $Y \equiv I+\varpi^{n} u B \bmod \varpi^{n+1}$. On peut bien sûr refaire ce calcul en remplaçant $B$ par $u^{-1} B$ et conclure. La surjectivité de $\pi_{e+1}$ suffit donc pour entraîner $H=G(\mathcal{O})$ si $p \geq e+2$ (resp. la surjectivité de $\pi_{m}$ avec $m \geq(e p+1) /(p-1)$ pour $p \leq e)$.

Si maintenant $n=e$, on reprend le calcul en supposant d'abord que $\pi_{1}\left(B^{2}\right)=0 \in \mathcal{L}$. Observons que, dans ce cas, $B^{2 j} \equiv 0 \bmod \varpi^{j}$ donc

$$
\begin{aligned}
& (B+\varpi C)^{p} \\
& =B^{p}+\varpi\left(B^{p-1} C+\cdots\right)+\cdots+\varpi^{r}\left(\cdots+B^{j_{0}} C B^{j_{1}} C \cdots C B^{j_{s}}\right)+\cdots+\varpi^{p} C^{p}
\end{aligned}
$$

avec $B^{p} \equiv 0 \bmod \varpi^{(p-1) / 2}$ et $\varpi^{r} B^{j_{0}} C B^{j_{1}} C \ldots C B^{j_{s}} \equiv 0 \bmod \varpi^{m}$ avec

$$
m \geq r+\left[\frac{j_{0}}{2}\right]+\cdots+\left[\frac{j_{s}}{2}\right] \geq r+\sum_{i=0}^{s}\left(\frac{j_{i}}{2}-\frac{1}{2}\right)=r+\frac{p-r}{2}-\frac{s+1}{2} \geq \frac{p-1}{2} .
$$


Si maintenant $Z=I+B+\varpi C$ et $Y=Z^{p}$ alors

$$
\begin{aligned}
Y & =I+p(B+\varpi C)+\sum_{h=2}^{p-1}\left(\begin{array}{l}
p \\
h
\end{array}\right) \varpi^{h}(B+\varpi C)^{p-h}+(B+\varpi C)^{p} \\
& \equiv I+p B+(B+\varpi C)^{p} \quad \bmod \varpi^{e+1} .
\end{aligned}
$$

La condition $(p-1) / 2 \geq e+1$ équivaut à $p \geq 2 e+3$. On trouve ainsi un élément $Y \in$ $H$ tel que $Y=I+\varpi^{e} B \bmod \varpi^{e+1}$. Pour le cas général, on aura $B=B_{1}+\cdots+B_{s}$ avec $\pi_{1}\left(B_{i}^{2}\right)=0 \in \mathcal{L}$ et $I+\varpi^{e} B \equiv\left(I+\varpi^{e} B_{1}\right) \cdots\left(I+\varpi^{e} B_{s}\right) \bmod \varpi^{e+1}$, ce qui permet de conclure.

Lemme 2.7 [Hindry et Ratazzi 2012, lemme 2.8]. Soit $d \geq 1$ un entier, et pour tout $i \in\{1, \ldots, d\}$, soient $t_{i} \geq 1$ des entiers. Pour $i \leq d$ et $j \leq t_{i}$, on se donne également des entiers $a_{i j}$ et $b_{i j}$, strictement positifs. On a l'égalité

$$
\sup _{\substack{m_{i 1} \geq \cdots \geq m_{i t_{i}} \\ 1 \leq i \leq d}}\left\{\frac{\sum_{i=1}^{d} \sum_{j=1}^{t_{i}} a_{i j} m_{i j}}{\sum_{i=1}^{d} \sum_{j=1}^{t_{i}} b_{i j} m_{i j}}\right\}=\max _{\substack{1 \leq h_{i} \leq t_{i} \\ 1 \leq i \leq d}}\left\{\frac{\sum_{i=1}^{d} \sum_{j=1}^{h_{i}} a_{i j}}{\sum_{i=1}^{d} \sum_{j=1}^{h_{i}} b_{i j}}\right\},
$$

le sup dans le membre de gauche étant pris sur les entiers $m_{i j}$ ordonnés pour $1 \leq i \leq d$ par $m_{i 1} \geq \cdots \geq m_{i t_{i}}$ et tels que $m_{i 1} \neq 0$.

\section{Représentations et accouplements $\lambda$-adiques}

L'étude de la représentation galoisienne adélique se ramène essentiellement à l'étude des représentations $\ell$-adiques, grâce au résultat suivant dû à Serre.

Définition 3.1. Une famille de représentations $\left(\rho_{i}\right)_{i \in I}: G_{K} \rightarrow \prod_{i \in I} \operatorname{GL}\left(V_{i}\right)$ indexée par un ensemble $I$, est dite indépendante si

$$
\left(\rho_{i}\right)_{i \in I}\left(G_{K}\right)=\prod_{i \in I}\left(\rho_{i}\left(G_{K}\right)\right) .
$$

Les $\left(\rho_{i}\right)_{i \in I}$ sont presque indépendantes s'il existe une extension finie $K^{\prime} / K$ telles que les restrictions à $G_{K^{\prime}}$ sont indépendantes.

Proposition 3.2 [Serre 1986c]. Les représentations $\ell$-adiques associées à une variété abélienne A sur un corps de nombres $K$ sont presque indépendantes.

Démonstration. C'est le théorème 1 de [Serre 1986c], cf. également [Serre 2013, théorème 1 et paragraphe 3.1 ].

Cet énoncé se traduit concrètement en disant que, quitte à remplacer $K$ par une extension finie, pour tous premiers $\ell_{1}, \ell_{2}$ distincts, les extensions $K\left(A\left[\ell_{1}^{\infty}\right]\right) / K$ et $K\left(A\left[\ell_{2}^{\infty}\right]\right) / K$ sont linéairement disjointes.

Nous utiliserons en parallèle les décompositions $\ell$-adiques et $\lambda$-adiques correspondant aux types I et II ; ces décompositions s'écrivent pour tout $\ell$ au niveau des 
$\mathbb{Q}_{\ell}$-représentations $V_{\ell}(A)$ et pour $\ell$ assez grand (hors d'un ensemble fini de $\ell$ ) pour les $\mathbb{Z}_{\ell}$-représentations $T_{\ell}(A)$.

Modules de Tate, représentations $\ell$-adiques et $\lambda$-adiques. Soit $\ell$ un premier quelconque. On considère, dans toute la suite de cette section 3 , une variété abélienne $A / K$ géométriquement simple de type I ou II, telle que $\operatorname{End}_{K}(A)=\operatorname{End}_{\bar{K}}(A)$ et telle que $\operatorname{End}_{K}(A)$ est un ordre maximal de $D:=\operatorname{End}_{K}(A) \otimes_{\mathbb{Z}} \mathbb{Q}$. Nous noterons $E$ le centre de $D$.

On a la décomposition suivante : $\mathcal{O}_{\ell}=\prod_{\lambda \mid \ell} \mathcal{O}_{\lambda}$, où $\mathcal{O}_{\lambda}$ est l'anneau des entiers du complété $E_{\lambda}$ de $E$ pour la place $\lambda$. En notant $f(\lambda):=\left[E_{\lambda}: \mathbb{Q}_{\ell}\right]$ et $e(\lambda)$ le degré de ramification de $\lambda \mid \ell$, on a $\sum_{\lambda \mid \ell} e(\lambda) f(\lambda)=e=[E: \mathbb{Q}]$.

Le module de Tate $\ell$-adique, $T_{\ell}(A)$, est muni d'une action de $\mathcal{O}_{\ell}$ et se décompose en

$$
T_{\ell}(A)=\prod_{\lambda \mid \ell} \mathcal{T}_{\lambda}, \quad \text { où } \mathcal{T}_{\lambda}:=T_{\ell}(A) \otimes_{\mathcal{O}_{\ell}} \mathcal{O}_{\lambda}
$$

En inversant $\ell$ on obtient les espaces $V_{\ell}(A)$ et $V_{\lambda}$ à partir de $T_{\ell}(A)$ et de $\mathcal{T}_{\lambda}$ :

$$
V_{\ell}(A):=T_{\ell}(A) \otimes_{\mathbb{Z}_{\ell}} \mathbb{Q}_{\ell} \quad \text { et } \quad V_{\lambda}:=\mathcal{T}_{\lambda} \otimes_{\mathcal{O}_{\lambda}} E_{\lambda}
$$

La représentation $\ell$-adique étant $\mathcal{O}_{E}$-linéaire, elle se décompose diagonalement selon les $\lambda$ divisant $\ell$ en représentations $\lambda$-adiques (cf. [Ribet 1976, paragraphe II]) :

$$
\rho_{\ell^{\infty}}=\left(\prod_{\lambda \mid \ell} \rho_{\lambda \infty}\right): G_{K} \rightarrow \prod_{\lambda \mid \ell} \operatorname{Aut}\left(\mathcal{T}_{\lambda}\right)
$$

La représentation $\ell$-adique modulo $\ell$ étant $\mathcal{O}_{E} / \ell \mathcal{O}_{E}$-linéaire, elle se décompose par réduction modulo $\ell$ diagonalement selon les $\lambda$ en représentations $\lambda$-adiques :

$$
\rho_{\ell}=\left(\prod_{\lambda \mid \ell} \rho_{\lambda}\right): G_{K} \rightarrow \operatorname{Aut}_{\mathcal{O}_{\ell} / \ell \mathcal{O}_{\ell}}(A[\ell])=\prod_{\lambda \mid \ell} \operatorname{Aut}_{\mathcal{O}_{\lambda} / \ell \mathcal{O}_{\lambda}}\left(\mathcal{T}_{\lambda} / \ell \mathcal{T}_{\lambda}\right) .
$$

Nous noterons dans toute la suite $G_{\lambda}$ le groupe de Galois correspondant à $\rho_{\lambda}$ (il est a priori à valeurs dans $\left.\mathrm{GL}_{2 h}\left(\mathcal{O}_{\lambda} / \ell \mathcal{O}_{\lambda}\right)\right)$. La même chose vaut au niveau $\ell$-adique et on sait (cf., par exemple, [Chi 1992, p. 319]) que ces représentations $\lambda$-adiques sont munies naturellement d'un accouplement de Weil $\lambda$-adique provenant de l'accouplement $\ell$-adique. Nous rappelons ceci dans le paragraphe suivant.

Accouplements $\ell$-adique et $\lambda$-adique. Nous supposons ici de plus que $A$ est polarisée par une polarisation $\phi$ et que $\ell$ est un premier ne divisant pas $\operatorname{deg}(\phi)$.

Rappelons la construction de l'accouplement $\lambda$-adique (cf. [Banaszak et al. 2006, paragraphes 3 et 4], par exemple). On commence pour cela par l'accouplement de Weil $\ell$-adique usuel :

$$
\phi_{\ell^{\infty}}: T_{\ell}(A) \times T_{\ell}(A) \rightarrow \mathbb{Z}_{\ell}(1)=\lim _{\longleftarrow} \mu_{\ell^{m}} .
$$


L'accouplement usuel de Weil est non dégénéré (modulo $\ell^{n}$ pour tout $n \geq 1$ ) car $\ell$ ne divise pas $\operatorname{deg}(\phi)$. De plus, si $\dagger$ désigne l'involution de $\operatorname{Rosati} \operatorname{sur} \operatorname{End}^{0}(A)$ associée à la polarisation définissant l'accouplement on aura $\phi_{\ell}(a x, y)=\phi_{\ell^{\infty}}\left(x, a^{\dagger} y\right)$ pour $x, y \in T_{\ell}(A)$ et $a \in \mathcal{O}_{\ell}$.

Lemme 3.3. Notons $\mathcal{O}_{\ell}^{\star}$ le dual de $\mathcal{O}_{\ell}$ pour la dualité donnée par la trace $\operatorname{Tr}_{E_{\ell} / \mathbb{Q}_{\ell}}$. Il existe un unique accouplement $\mathcal{O}_{\ell}$-linéaire, $\phi_{\ell^{\infty}}^{\star}: T_{\ell}(A) \times T_{\ell}(A) \rightarrow \mathcal{O}_{\ell}^{\star}(1)$, tel que

$$
\operatorname{Tr}_{E_{\ell} / \mathbb{Q}_{\ell}}\left(\phi_{\ell^{\infty}}^{\star}\right)=\phi_{\ell^{\infty}} .
$$

Démonstration. Il s'agit essentiellement du lemme 3.1 de [Banaszak et al. 2006] (cf. aussi [Deligne et al. 1982, Sublemma 4.7, p.55]). Le preuve est la suivante : il s'agit de vérifier que le morphisme

$$
\operatorname{Hom}_{\mathcal{O}_{\ell}}\left(T_{\ell}(A) \otimes_{\mathcal{O}_{\ell}} T_{\ell}(A), \mathcal{O}_{\ell}^{\star}\right) \rightarrow \operatorname{Hom}_{\mathbb{Z}_{\ell}}\left(T_{\ell}(A) \otimes_{\mathcal{O}_{\ell}} T_{\ell}(A), \mathbb{Z}_{\ell}\right),
$$

donné par la trace, est un isomorphisme. Or ces deux objets sont des $\mathbb{Z}_{\ell}$-modules libres de même rang et la preuve du lemme 3.1 de [Banaszak et al. 2006] donne la surjectivité.

Hypothèse. On suppose dans la fin de ce paragraphe que $\ell$ est de plus non ramifié dans $E / \mathbb{Q}$, i.e., que $\mathcal{O}_{\ell}^{\star}=\mathcal{O}_{\ell}$.

On a dans ce cas l'accouplement $\mathcal{O}_{\ell}$-linéaire $\phi_{\ell^{\infty}}^{\star}: T_{\ell}(A) \times T_{\ell}(A) \rightarrow \mathcal{O}_{\ell}(1)$. Par projection on construit alors l'accouplement $\lambda$-adique, $\mathcal{O}_{\lambda}$-linéaire, de la façon suivante :

$$
\phi_{\lambda \infty}: \mathcal{T}_{\lambda} \times \mathcal{T}_{\lambda} \rightarrow \mathcal{O}_{\lambda}(1)
$$

tel que $\phi_{\ell^{\infty}}^{\star}(x, y) \otimes 1=\phi_{\lambda \infty}(x \otimes 1, y \otimes 1)$.

Tout comme l'accouplement $\ell$-adique, l'accouplement $\lambda$-adique est Galois équivariant :

$$
\forall \sigma \in G_{K}, \forall x, y \in \mathcal{T}_{\lambda}, \quad \phi_{\lambda \infty}(\sigma x, \sigma y)=\phi_{\lambda \infty}(x, y)^{\sigma},
$$

l'action de Galois à gauche se faisant via la représentation $\lambda$-adique, et à droite, via le caractère cyclotomique $\ell$-adique usuel.

Nous noterons enfin $\phi_{\ell^{\infty}}^{0}$ et $\phi_{\lambda \infty}^{0}$ les accouplements $\ell$-adiques et $\lambda$-adiques, définis de manière similaires sur $V_{\ell}(A)$ et $V_{\lambda}$, à valeurs respectivement dans $E_{\ell}$ et $E_{\lambda}$. Ils sont définis sans restriction pour tout $\ell$.

Galois pour les variétés de type I et II. Soit $\ell$ un premier quelconque.

Dans le cas de type I, les $\left(V_{\lambda}, \phi_{\lambda^{\infty}}^{0}\right)$ fournissent des représentations irréductibles symplectiques. Dans le cas de type II, on a une décomposition plus fine (cf. [Chi 1990; Milne 1999; Banaszak et al. 2006]) :

$$
V_{\lambda}=W_{\lambda}(A) \oplus W_{\lambda}(A),
$$


où $\left(W_{\lambda}(A), \phi_{\lambda \infty \mid W_{\lambda}(A)}^{0}\right)$ est maintenant irréductible symplectique. Cependant, comme nous le détaillons plus loin, cette décomposition, dans le cas de type II, nécessite, pour le nombre fini de premiers $\ell$ "ramifiés" pour l'algèbre de quaternions, d'étendre les scalaires à une extension quadratique.

Nous avons $V_{\lambda}:=W_{\lambda}(A) \oplus W_{\lambda}(A)$ si $A$ est de type II et nous posons $W_{\lambda}(A):=V_{\lambda}$ si $A$ est de type I. Autrement dit en notant $d=1$ si $A$ est de type I et $d=2$ si $A$ est de type II, nous aurons toujours

$$
V_{\lambda}=W_{\lambda}(A)^{d},
$$

et dans tous les cas, la représentation $W_{\lambda}(A)$ est irréductible, symplectique et, lorsque $A$ est de type I (resp. de type II), le module $V_{\ell}(A)$ est isomorphe à $\prod_{\lambda} W_{\lambda}(A)$ (resp. à la somme de deux copies de ce produit). On pose ensuite dans tout les cas

$$
T_{\lambda}(A):=\mathcal{T}_{\lambda} \cap W_{\lambda}(A) .
$$

Définition 3.4. Nous noterons dans la suite $S_{\mathrm{ex}}(A)$ l'ensemble fini des $\ell$ divisant le degré de la polarisation fixée $\phi$ de $A$, des $\ell$ ramifiés dans $\mathcal{O}_{E}$ et, dans le cas de type II, des $\ell$ tels que l'algèbre de quaternions $D$ est non décomposée en au moins un $\lambda \mid \ell$.

Au niveau des $\mathbb{Z}_{\ell}$-modules, la décomposition perdure, au moins pour $\ell$ hors de $S_{\mathrm{ex}}(A)$. Nous rassemblons ces énoncés dans la proposition suivante.

Proposition 3.5. Soit $W_{\lambda}(A)$ le $E_{\lambda}$-module galoisien symplectique défini ci-dessus, qu'on identifie à un sous-module de $V_{\ell}(A)$.

(1) La représentation $W_{\lambda}(A)$ est irréductible et symplectique.

(2) Si A est de type I, on a une décomposition $V_{\ell}(A)=\prod_{\lambda \mid \ell} W_{\lambda}(A)$, et si A est de type II, $V_{\ell}(A)=\prod_{\lambda \mid \ell}\left(W_{\lambda}(A) \oplus W_{\lambda}(A)\right)$. Toutefois, dans le cas de type II et pour les premiers ramifiés de l'algèbre de quaternions, cette décomposition ne s'obtient qu'après tensorisation par une extension quadratique de E.

(3) Pour $\ell \notin S_{\mathrm{ex}}(A)$, on a une décomposition analogue pour les $\mathcal{O} \otimes \mathbb{Z}_{\ell}$-modules: $T_{\ell}(A)=\prod_{\lambda \mid \ell} T_{\lambda}(A)$ si $A$ est de type $I$, et $T_{\ell}(A)=\prod_{\lambda}\left(T_{\lambda}(A) \oplus T_{\lambda}(A)\right)$ si $A$ est de type II.

Démonstration. Pour $A$ de type I, voir [Ribet 1976]. Pour $A$ de type II, cet énoncé est prouvé pour $\ell$ assez grand sur $\mathbb{Q}_{\ell}$ dans [Chi 1990] et sur $\mathbb{Z}_{\ell}$ dans [Banaszak et al. 2006], le point essentiel étant l'identification de $\operatorname{End}^{0}(A) \otimes \mathbb{Q}_{\ell}$ à un produit d'algèbres de matrices $\prod_{\lambda} M_{2}\left(E_{\lambda}\right)$, ce qui est possible justement quand $\ell$ n'est pas ramifié. L'algèbre de quaternions est toujours déployée sur une extension quadratique et l'on peut donc se ramener au cas précédent après tensorisation par une telle extension; plus précisément, en choisissant $F$ extension quadratique de $E$ telle que $D \otimes_{E} F \cong M_{2}(F)$ et en notant $F_{\lambda}:=E_{\lambda} \otimes_{E} F$ et $V_{\ell} \otimes_{E} F=\prod_{\lambda} V_{\lambda, F}$, 
chaque $V_{\lambda, F}$ est un module sur $D \otimes_{E} F_{\lambda} \cong M_{2}\left(F_{\lambda}\right)$ et on peut alors imiter le procédé décrit dans [loc. cit.]. Remarquons que la preuve sur $\mathbb{Q}_{\ell}$ pour tout $\ell$ peut aussi être extraite de [Milne 1999].

Définition 3.6. Définissons la dimension relative de $A$ simple avec $D:=\operatorname{End}^{0}(A)$ et $E$ le centre de $D$, par la formule :

$$
\operatorname{dimrel}(A):=\frac{\operatorname{dim} A}{[E: \mathbb{Q}] \sqrt{[D: E]}} .
$$

Ainsi, si $e=[E: \mathbb{Q}]$, la dimension relative $h$ d'une variété abélienne de dimension $g$ de type I (resp. de type II) est $h=g / e$ (resp. $h=g /(2 e)$ ).

On a alors dans le cas I et II une représentation irréductible symplectique de dimension $2 h$, et une inclusion

$$
H_{\ell, A} \subset \prod_{\lambda \mid \ell} \operatorname{Sp}\left(W_{\lambda}(A), \phi_{\lambda \infty}^{0}\right) \cong \prod_{\lambda \mid \ell} \operatorname{Sp}_{2 h, E_{\lambda}},
$$

qu'il convient de mettre en parallèle avec l'inclusion

$$
\operatorname{Hdg}(A)_{\mathbb{Q}_{\ell}} \subset\left(\operatorname{Res}_{E / \mathbb{Q}} \operatorname{Sp}_{2 h, E}\right)_{\mathbb{Q}_{\ell}} .
$$

Nous allons nous placer dans le cas générique où l'on a égalité dans les deux inclusions précédentes (la première impliquant d'ailleurs la seconde puisque $H_{\ell, A} \subset$ $\left.\operatorname{Hdg}(A)_{\mathbb{Q}_{\ell}}\right)$. Le théorème suivant précise des conditions où l'on sait que l'égalité voulue est toujours vraie.

Théorème 3.7 [Banaszak et al. 2006; Pink 1998; Hall 2011]. Soit A/K une variété abélienne de type I ou II, de dimension relative $h ;$ notons $E$ le centre de $\operatorname{End}^{0}(A)$. Supposons de plus l'une des trois conditions suivantes réalisée:

(1) L'entier h est impair ou égal à 2.

(2) On a $E=\mathbb{Q}$ et h n'appartient pas à l'ensemble exceptionnel $\Sigma$ défini par l'équation (1).

(3) La variété abélienne A est de type I (resp. II) et possède une place de mauvaise réduction semi-stable avec dimension torique e (resp. avec dimension torique $2 e$ ).

On a alors $H_{\ell, A}=\prod_{\lambda} \mathrm{Sp}_{2 h, E_{\lambda}}$.

Démonstration. Le résultat est démontré dans [Banaszak et al. 2006] sous l'hypothèse de l'alinéa (1) (cf. [Lombardo 2016, Remark 2.25] pour le cas $h=2$ ); le résultat est démontré explicitement dans [Pink 1998] sous l'hypothèse de l'alinéa (2), pour une variété abélienne de type I, mais on peut extraire de [Pink 1998] une preuve pour le type II ; nous indiquons comment en appendice de cet article (cf. section 10). Le résultat est démontré dans [Hall 2011] sous l'hypothèse de l'alinéa (3), pour 
une variété abélienne de type I vérifiant $e=1$; nous indiquons en appendice de cet article comment étendre les arguments de [Hall 2011] aux cas énoncés.

Variantes $\lambda$-adiques modulo $\lambda^{n}$. On suppose ici que $\ell$ est un premier quelconque. Soit $n \geq 1$ un entier. On a par réduction modulo $\ell^{n}$,

$$
A\left[\ell^{n}\right]=T_{\ell}(A) \otimes_{\mathbb{Z}_{\ell}} \mathbb{Z} / \ell^{n} \mathbb{Z}=T_{\ell}(A) / \ell^{n} T_{\ell}(A) .
$$

De même par réduction modulo $\lambda^{n}$, on voit que

$$
A\left[\lambda^{n}\right]:=\mathcal{T}_{\lambda} \otimes_{\mathcal{O}_{\lambda}} \mathcal{O}_{\lambda} / \lambda^{n}=\mathcal{T}_{\lambda} / \lambda^{n} \mathcal{T}_{\lambda}
$$

On pose ensuite

$$
T_{\lambda}\left[\lambda^{n}\right]:=T_{\lambda}(A) \otimes_{\mathcal{O}_{\lambda}} \mathcal{O}_{\lambda} / \lambda^{n}=T_{\lambda}(A) / \lambda^{n} T_{\lambda}(A)
$$

En utilisant que $\ell^{n} \mathcal{O}_{\lambda}=\lambda^{e(\lambda) n}$ on voit que par réduction modulo $\ell^{n}$ on obtient :

$$
A\left[\ell^{n}\right]=T_{\ell}(A) / \ell^{n} T_{\ell}(A)=\prod_{\lambda \mid \ell} \mathcal{T}_{\lambda} / \ell^{n} \mathcal{T}_{\lambda},
$$

et

$$
T_{\lambda}(A) / \ell^{n} T_{\lambda}(A)=T_{\lambda}(A) \otimes_{\mathcal{O}_{\lambda}} \mathcal{O}_{\lambda} / \lambda^{e(\lambda) n}=T_{\lambda}\left[\lambda^{e(\lambda) n}\right]
$$

Soit $\pi_{\lambda}$ une uniformisante de $\lambda$ dans $O_{\lambda}$ ( $\pi_{\lambda}=\ell$ dans le cas non ramifié). Pour tout entier $n \geq 0$, les applications

$$
i_{n}: T_{\lambda}\left[\lambda^{n}\right] \rightarrow T_{\lambda}\left[\lambda^{n+1}\right], \quad x \bmod \lambda^{n} T_{\lambda}(A) \mapsto \pi_{\lambda} x \bmod \lambda^{n+1} T_{\lambda}(A)
$$

sont des morphismes, bien définis, injectifs. En prenant le système inductif qu'ils forment, on note $T_{\lambda}\left[\lambda^{\infty}\right]$ la limite.

Supposons dans la fin de ce paragraphe que $\ell$ est non ramifié. Dans ce cas le $\mathcal{O}_{\ell} / \ell^{n} \mathcal{O}_{\ell}$-module $A\left[\ell^{n}\right]$ se décompose en le produit des $\mathcal{O}_{\lambda} / \lambda^{n}$-modules $T_{\lambda}\left[\lambda^{n}\right]$ dans le cas de type I, et, pour $\ell \notin S_{\text {ex }}(A)$, en le produit des $T_{\lambda}\left[\lambda^{n}\right] \oplus T_{\lambda}\left[\lambda^{n}\right]$ dans le cas de type II. De plus, par projection modulo $\ell^{n}$ (ou ce qui revient au même ici, modulo $\lambda^{n}$ ), on obtient :

$\phi_{\lambda^{n}}: T_{\lambda}\left[\lambda^{n}\right] \times T_{\lambda}\left[\lambda^{n}\right] \rightarrow \mathcal{O}_{\lambda} / \ell^{n} \mathcal{O}_{\lambda}(1), \quad$ qui vérifie $\quad \phi_{\lambda^{n}}(\ell x, \ell y)=\phi_{\lambda^{n+1}}(x, y)^{\ell}$.

Tout comme l'accouplement $\lambda$-adique, on voit par réduction modulo $\ell^{n}$ que les accouplements $\phi_{\lambda^{n}}$ sont Galois équivariants, l'action de Galois à gauche se faisant via la représentation $\lambda$-adique, et à droite, via le caractère cyclotomique $\ell$-adique usuel. 


\section{Modules isotropes}

On considère dans ce paragraphe une variété abélienne $A / K$ géométriquement simple de type I ou II, telle que $\operatorname{End}_{K}(A)=\operatorname{End}_{\bar{K}}(A)$ et telle que $\operatorname{End}_{K}(A)$ est un ordre maximal de $\operatorname{End}_{K}(A) \otimes_{\mathbb{Z}} \mathbb{Q}$. Soit par ailleurs $\ell$ un premier et $\lambda$ une place au dessus de $\ell$. Notons $\pi_{\lambda}$ une uniformisante de $\lambda$. On suppose ici que $\ell$ est tel que la condition suivante (qui exclut un nombre fini de premiers) est réalisée : on a un accouplement bilinéaire alterné, non dégénéré sur $T_{\lambda}(A)$ (qui est un $\mathcal{O}_{\lambda}$-module libre de rang $2 h$ ) et sur le $\mathbb{F}_{\lambda}$-espace vectoriel $T_{\lambda}[\lambda]=T_{\lambda}(A) / \lambda T_{\lambda}(A)$.

Définition 4.1. Soit $H \subset T_{\lambda}\left[\lambda^{\infty}\right]$ un sous-groupe fini. Nous dirons que $H$ est totalement isotrope si pour tous points $P, Q$ de $H \subset T_{\lambda}\left[\lambda^{n}\right]$, on a

$$
\phi_{\lambda^{n}}(P, Q)=1
$$

où $\phi_{\lambda^{n}}$ désigne l'accouplement sur $T_{\lambda}\left[\lambda^{n}\right]$.

Notons que si $H$ est totalement isotrope au sens précédent, alors son sous-groupe des points de $\lambda$-torsion est totalement isotrope dans le $\mathbb{F}_{\lambda}$-espace vectoriel $T_{\lambda}[\lambda]$. On retrouve avec cette définition les deux lemmes suivants dont les preuves se reprennent mot pour mot du paragraphe 3.1 de [Hindry et Ratazzi 2012] en remplaçant $\mathbb{Z} \operatorname{par} \mathcal{O}_{\lambda}$ et $\ell$ par $\lambda$.

Lemme 4.2. Soit $\left(e_{1}, \ldots, e_{h}\right)$ une base d'un sous- $\mathcal{O}_{\lambda}$-module isotrope maximal $H_{\infty}$ de $T_{\lambda}(A)$. Il existe un supplémentaire $H_{\infty}^{\prime}$ isotrope maximal et une base $\left(e_{h+1}, \ldots, e_{2 h}\right)$ de celui-ci de sorte que dans la décomposition $T_{\lambda}(A)=H_{\infty} \oplus H_{\infty}^{\prime}$ selon la base $\left(e_{1}, \ldots, e_{2 h}\right)$, la forme symplectique s'écrit comme la forme canonique

$$
J=\left(\begin{array}{cc}
0 & I_{h} \\
-I_{h} & 0
\end{array}\right)
$$

Lemme 4.3. Soit $n \geq 1$ et $H \subset T_{\lambda}\left[\lambda^{n}\right]$ un sous-groupe fini, totalement isotrope. Notons $\operatorname{pr}_{n}: T_{\lambda}(A) \rightarrow T_{\lambda}\left[\lambda^{n}\right]$ la projection canonique modulo $\lambda^{n}$. Il existe un sous-groupe totalement isotrope $H_{\mathrm{ti}}$ de $T_{\lambda}\left[\lambda^{n}\right]$, contenant $H$ et de même exposant et il existe un sous- $\mathcal{O}_{\lambda}$-module, $H_{\infty}$ de $T_{\lambda}(A)$, totalement isotrope, tel que $\operatorname{pr}_{n}\left(H_{\infty}\right)=H_{\mathrm{ti}}$.

Remarque 4.4. Notons que dans [Hindry et Ratazzi 2012] la version correspondante du lemme précédent ne mentionne pas que l'on peut choisir $H_{\mathrm{ti}}$ de même exposant que $H$. Toutefois la construction même de ce $H_{\mathrm{ti}}$ fournie dans la preuve du lemme 3.7 de [Hindry et Ratazzi 2012] donne immédiatement cette information supplémentaire. 


\section{Propriété $\mu$, version $\lambda$-adique}

On considère dans ce paragraphe une variété abélienne $A / K$ géométriquement simple de type I ou II, telle que $\operatorname{End}_{K}(A)=\operatorname{End}_{\bar{K}}(A)$ et telle que $\operatorname{End}_{K}(A)$ est un ordre maximal de $\operatorname{End}_{K}(A) \otimes_{\mathbb{Z}} \mathbb{Q}$. On suppose par ailleurs ici que $\ell \notin S_{\mathrm{ex}}(A)$.

Propriété $\mu$. Étant donné un sous-groupe $H$ fini de $T_{\lambda}\left[\lambda^{\infty}\right]$, nous introduisons à présent l'invariant suivant :

$m_{1}(H):=\max \left\{k \in \mathbb{N} \mid \exists n \geq 0, \exists P, Q \in H\right.$ d'ordre $\ell^{n}, \phi_{\lambda^{n}}(P, Q)$ est d'ordre $\left.\ell^{k}\right\}$.

Dire que $H$ est totalement isotrope équivaut à dire que $m_{1}(H)=0$. De plus on peut noter que, sur la définition, il est évident que $m_{1}(H)$ est supérieur à la valeur $m$ suivante :

$$
m(H):=\max \left\{k \in \mathbb{N} \mid \exists P, Q \in H \text { d'ordre } \ell^{k}, \phi_{\lambda^{k}}(P, Q) \text { est d'ordre } \ell^{k}\right\} .
$$

Lorsque $H$ est de la forme $T_{\lambda}\left[\lambda^{n}\right]$, nous allons montrer que

$$
m_{1}\left(T_{\lambda}\left[\lambda^{n}\right]\right)=m\left(T_{\lambda}\left[\lambda^{n}\right]\right)=n .
$$

Définition 5.1. Nous appelons propriété $\mu$ pour une variété abélienne le fait d'avoir, pour tout sous-groupe fini $H \subset T_{\lambda}\left[\lambda^{\infty}\right]$, l'égalité à indice fini près, uniformément en $(\ell, H)$ :

$$
K\left(\mu_{\ell^{m_{1}(H)}}\right) \asymp K(H) \cap K\left(\mu_{\ell}\right) .
$$

Propriété $\mu$ pour $\boldsymbol{T}_{\lambda}\left[\lambda^{n}\right]$. Soit $n \geq 1$ un entier. La propriété $\mu$ pour $T_{\lambda}\left[\lambda^{n}\right]$ découle essentiellement formellement de la propriété $\mu$ pour $A\left[\ell^{n}\right]$ et du fait que le multiplicateur mult $t_{\lambda}\left(\rho_{\lambda}(\sigma)\right)$ est $\chi_{\ell}(\sigma)$. Plus précisément, on sait que concernant l'image de la représentation $\lambda$-adique résiduelle $\rho_{\lambda}$ (à valeur dans $\mathbb{F}_{\lambda}$ ), on a

Proposition 5.2. Soit A de type I ou II et pleinement de type Lefschetz. On a les (presque) égalités suivantes, le produit portant sur les places $\lambda$ au dessus de $\ell$ dans l'anneau des entiers $\mathcal{O}_{E}$ de $E$ :

(1) $\left[\rho_{\ell}\left(G_{K}\right), \rho_{\ell}\left(G_{K}\right)\right] \stackrel{\circ}{=} \prod_{\lambda \mid \ell} \operatorname{Sp}_{2 h}\left(\mathbb{F}_{\lambda}\right) \stackrel{\circ}{=} \operatorname{Sp}_{2 h}\left(\mathcal{O}_{E} / \ell \mathcal{O}_{E}\right)$.

(2) $\rho_{\lambda}\left(G_{K}\right) \stackrel{\circ}{=}\left\{x \in \mathrm{GSp}_{2 h}\left(\mathbb{F}_{\lambda}\right) \mid \operatorname{mult}(x) \in \mathbb{F}_{\ell}^{\times}\right\}$.

(3) $\left[\rho_{\ell \infty}\left(G_{K}\right), \rho_{\ell \infty}\left(G_{K}\right)\right] \stackrel{\circ}{=} \operatorname{Hdg}(A)\left(\mathbb{Z}_{\ell}\right)=\prod_{\lambda \mid \ell} \operatorname{Sp}_{2 h}\left(\mathcal{O}_{\lambda}\right)$.

(4) $\rho_{\lambda \infty}\left(G_{K}\right) \stackrel{\circ}{=}\left\{x \in \operatorname{GSp}_{2 h}\left(\mathcal{O}_{\lambda}\right) \mid \operatorname{mult}(x) \in \mathbb{Z}_{\ell}^{\times}\right\}$.

(5) $\rho_{\ell^{\infty}}\left(G_{K}\right) \stackrel{\circ}{=} \mathrm{MT}(A)\left(\mathbb{Z}_{\ell}\right)=\left\{\left(x_{\lambda}\right) \in \prod_{\lambda \mid \ell} \operatorname{GSp}_{2 h}\left(\mathcal{O}_{\lambda}\right) \mid \forall \lambda, \exists y \in \mathbb{Z}_{\ell}^{\times}, \operatorname{mult}\left(x_{\lambda}\right)=y\right\}$.

Démonstration. L'hypothèse que $A$ est de type Lefschetz signifie que $\operatorname{Hdg}(A)=$ $\operatorname{Res}_{E / \mathbb{Q}} \operatorname{Sp}_{E, 2 h}$ et $\operatorname{MT}(A)=\mathbb{G}_{m} \operatorname{Res}_{E / \mathbb{Q}} \operatorname{Sp}_{E, 2 h}$. L'hypothèse que $A$ est pleinement de Lefschetz signifie que l'image de Galois est d'indice fini dans $\operatorname{MT}(A)\left(\mathbb{Z}_{\ell}\right)$. Comme nous l'expliquons en appendice (théorème 10.1), ceci entraîne que cet 
indice est borné indépendamment de $\ell$. En particulier l'indice de $\rho_{\lambda}\left(G_{K}\right) \cap \operatorname{Sp}_{2 h}\left(\mathbb{F}_{\lambda}\right)$ est borné indépendamment de $\ell$, disons par $c$. Observons maintenant que $\operatorname{Sp}_{2 h}\left(\mathbb{F}_{\ell^{m}}\right)$ ne possède pas de sous-groupe d'indice "petit" (ceci se voit en appliquant les lemmes 2.5 et 2.13 de [Hindry et Ratazzi 2012]), c'est-à-dire que, pour $\ell \geq \ell_{0}=\ell_{0}(c)$, un sous-groupe d'indice inférieur à $c$ est égal au groupe $\operatorname{Sp}_{2 h}\left(\mathbb{F}_{\lambda}\right)$ tout entier. D'après le lemme 2.6 nous pouvons conclure que, pour $\ell \geq \ell_{0}$, nous avons $\left[\rho_{\lambda \infty}\left(G_{K}\right), \rho_{\lambda \infty}\left(G_{K}\right)\right]=\operatorname{Sp}_{2 h}\left(\mathcal{O}_{\lambda}\right)$. Ensuite en utilisant le fait que mult ${ }_{\lambda}\left(\rho_{\lambda \infty}(\sigma)\right)=$ $\chi_{\ell^{\infty}}(\sigma)$ et que le caractère cyclotomique est surjectif sur $\mathbb{Z}_{\ell}^{\times}$, toujours pour $\ell$ assez grand, on conclut que $\rho_{\ell^{\infty}}\left(G_{K}\right) \stackrel{\circ}{=} \mathrm{MT}(A)\left(\mathbb{Z}_{\ell}\right)$, comme annoncé. Les autres égalités s'en déduisent aisément.

On peut déduire de ces considérations l'observation suivante concernant la partie cyclotomique des extensions engendrées, valable pour $A$ pleinement de type Lefschetz, de type I ou II. On peut décrire la situation via la tour d'extensions suivante :

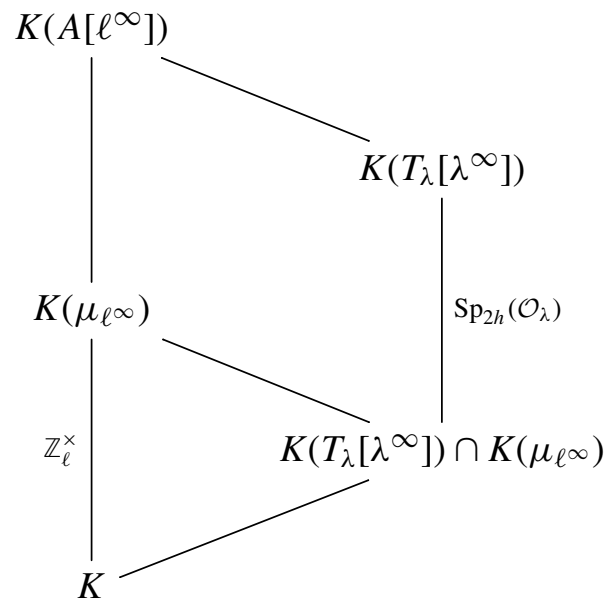

Nous résumons cela dans le corollaire suivant.

Corollaire 5.3. Soit A de type I ou II, et pleinement de type Lefschetz. On a les (presque) égalités suivantes:

$$
K\left(T_{\lambda}\left[\lambda^{\infty}\right]\right) \cap K\left(\mu_{\ell^{\infty}}\right) \stackrel{\circ}{=}\left(\mu_{\ell^{\infty}}\right),
$$

On a le même résultat en niveau fini par réduction modulo $\ell^{n}$.

Propriété $\mu$ pour $H \subset T_{\lambda}\left[\lambda^{n}\right]$.

Proposition 5.4. Soit $H$ un sous-groupe fini de $T_{\lambda}\left[\lambda^{\infty}\right]$. On a, uniformément en $(\ell, H)$, l'inégalité

$$
\left[K\left(\mu_{\ell^{m_{1}(H)}}\right): \mathbb{Q}\right] \ll\left[K(H) \cap K\left(\mu_{\ell^{\infty}}\right): \mathbb{Q}\right] .
$$


Démonstration. Soit $x, y \in H$ deux points de même ordre $\ell^{n}$ tels que $\phi_{\lambda^{n}}(x, y)$ est un élément d'ordre $\ell^{m_{1}(H)}$. Montrons que l'extension $K(x, y)$ contient "presque" $K\left(\mu_{\ell^{m_{1}(H)}}\right)$. Ces deux extensions sont des sous- $K$-extensions de $K\left(T_{\lambda}\left[\lambda^{n}\right]\right)$ et par la description du groupe de Galois de $K\left(T_{\lambda}\left[\lambda^{n}\right]\right) / K$, on voit que le groupe de Galois $G_{x, y}$ de $K\left(T_{\lambda}\left[\lambda^{n}\right]\right)$ sur $K(x, y)$ est donnée par la presque égalité suivante (valable pour tout $\ell$ assez grand),

$$
\begin{aligned}
G_{x, y} \stackrel{\circ}{=}\left\{\rho_{\lambda^{n}}(\sigma) \in \mathrm{GSp}_{2 h}\left(\mathcal{O}_{\lambda} / \ell^{n} \mathcal{O}_{\lambda}\right) \mid \sigma \in G_{K}, \sigma \cdot x=x, \sigma \cdot y=y,\right. & \text { et } \left.\chi \ell^{n}(\sigma) \in\left(\mathbb{Z} / \ell^{n} \mathbb{Z}\right)^{\times}\right\} .
\end{aligned}
$$

Soit donc $\sigma \in G_{K}$ tel que $\rho_{\lambda^{n}}(\sigma) \in G_{x, y}$. On a

$$
\phi_{\lambda^{n}}(x, y)=\phi_{\lambda^{n}}\left(\rho_{\lambda^{n}}(\sigma)(x), \rho_{\lambda^{n}}(\sigma)(y)\right)=\chi_{\ell^{n}}(\sigma) \phi_{\lambda^{n}}(x, y) .
$$

On en déduit que $\chi_{\ell^{n}}(\sigma)-1$ est un multiple de l'ordre de $\phi_{\lambda^{n}}(x, y)$ dans $\mathcal{O}_{\lambda} / \ell^{n} \mathcal{O}_{\lambda}$, autrement dit que $\chi_{\ell^{n}}(\sigma)=1 \bmod \ell^{m_{1}(H)}$. Or le groupe de Galois de $K\left(T_{\lambda}\left[\lambda^{n}\right]\right)$ sur $K\left(\mu_{\ell^{m_{1}(H)}}\right)$ est précisément constitué des $\rho_{\lambda^{n}}(\sigma)$ tels que $\chi_{\ell^{n}}(\sigma)=1 \bmod \ell^{m_{1}(H)}$. On en déduit le résultat.

Nous pouvons maintenant prouver la propriété $\mu$ proprement dite :

\section{Proposition 5.5. En notant}

$$
\delta(H):=\left(\mathbb{Z}_{\ell}^{\times}: \operatorname{mult}\left(G_{0}(H)\right)\right), \quad \text { où } G_{0}(H)=\operatorname{Gal}\left(K\left(A\left[\lambda^{\infty}\right]\right) / K(H)\right),
$$

on a, pour tout sous-groupe $H$ fini de $T_{\lambda}\left[\lambda^{\infty}\right]$, l'égalité à indice fini près, uniformément en $(\ell, H)$,

$$
\left[K(H) \cap K\left(\mu_{\ell^{\infty}}\right): K\right] \asymp \delta(H) .
$$

De plus, pour tout $H$ sous-groupe fini de $T_{\lambda}\left[\lambda^{\infty}\right]$, on a l'inclusion suivante, qui est une égalité à un indice fini près uniformément en $(\ell, H)$ :

$$
K(H) \cap K\left(\mu_{\ell^{\infty}}\right) \subset K\left(\mu_{\ell^{m_{1}(H)}}\right) \quad \text { et } \quad K(H) \cap K\left(\mu_{\ell^{\infty}}\right) \asymp K\left(\mu_{\ell^{m_{1}}(H)}\right) .
$$

Démonstration. On a la presque égalité $\operatorname{Gal}\left(K\left(T_{\lambda}\left[\lambda^{\infty}\right]\right) / K\right) \stackrel{\circ}{=} G_{\lambda}$ (introduit au lemme 2.2). Le groupe de Galois $\operatorname{Gal}\left(K\left(T_{\lambda}\left[\lambda^{\infty}\right]\right) / K\left(\mu_{\ell^{\infty}}\right)\right)$ s'identifie (c'est une presque égalité) alors avec $\mathrm{SG}_{\lambda}:=G_{\lambda} \cap \operatorname{Ker}\left(\right.$ mult). Alors $K(H) \cap K\left(\mu_{\ell \infty}\right)$ est la sous-extension fixée par le groupe $U$ engendré par $\mathrm{SG}_{\lambda}$ et $G_{0}(H)$. On voit immédiatement que le noyau de $G_{\lambda} \stackrel{\text { mult }}{\longrightarrow} \mathbb{Z}_{\ell}^{\times} \rightarrow \mathbb{Z}_{\ell}^{\times} / \operatorname{mult}\left(G_{0}(H)\right)$ est le groupe $U$, d'où le premier énoncé.

Passons maintenant à la seconde partie de la proposition. Commençons par considérer $H_{\infty}$ un sous-groupe isotrope maximal de $T_{\lambda}(A)$. Par le lemme 4.2, on peut supposer que dans une décomposition $T_{\lambda}(A)=H_{\infty} \oplus H_{\infty}^{\prime}$ la forme symplectique 
s'écrit comme la forme canonique $J$. On voit alors aisément que,

$$
\begin{aligned}
\operatorname{Gal}\left(K\left(T_{\lambda}\left[\lambda^{\infty}\right]\right) / K\left(H_{\infty}\right)\right) & \asymp\left\{M=\left(\begin{array}{cc}
I & * \\
0 & *
\end{array}\right) \in \operatorname{GSp}_{2 h}\left(\mathcal{O}_{\lambda}\right) \mid \operatorname{mult}(M) \in \mathbb{Z}_{\ell}^{\times}\right\} \\
& =\left\{M=\left(\begin{array}{cc}
I & S \\
0 & \alpha I
\end{array}\right) \mid \alpha \in \mathbb{Z}_{\ell}^{\times} \text {et } S \text { symétrique }\right\} .
\end{aligned}
$$

D'après le lemme 2.2, le groupe engendré par ce dernier groupe et par le groupe $\operatorname{Sp}_{2 h}\left(\mathcal{O}_{\lambda}\right) \stackrel{\circ}{G} \operatorname{Gal}\left(K\left(T_{\lambda}\left[\lambda^{\infty}\right]\right) / K\left(\mu_{\ell}\right)\right)$ est $\left\{x \in \mathrm{GSp}_{2 h}\left(\mathcal{O}_{\lambda}\right) \mid \operatorname{mult}(x) \in \mathbb{Z}_{\ell}^{\times}\right\}$tout entier. Ainsi $K\left(H_{\infty}\right) \cap K\left(\mu_{\ell}\right) \asymp K$. Si $H$ est un sous-groupe fini de $T_{\lambda}\left[\lambda^{\infty}\right]$ totalement isotrope, dans ce cas le lemme 4.3 et ce qui précède nous permettent de conclure : on a $K(H) \cap K\left(\mu_{\ell}\right) \asymp K$.

Soit maintenant $H$ un sous-groupe fini non isotrope de $T_{\lambda}\left[\lambda^{\infty}\right]$. Le groupe $\left[\ell^{m_{1}(H)}\right](H)$ est totalement isotrope. En effet si $P$ et $Q$ sont deux points d'ordre $\ell^{n}$ dans $H$, alors, par définition de $m_{1}(H)$,

$$
\phi_{\lambda^{n-m_{1}(H)}}\left(\ell^{m_{1}(H)} P, \ell^{m_{1}(H)} Q\right)=\phi_{\lambda^{n}}(P, Q)^{\ell^{m_{1}(H)}}=1 .
$$

En appliquant le lemme 4.3, on trouve donc un sous-groupe $H^{\prime}$ contenant $\left[\ell^{m_{1}}\right](H)$ de même exposant et il existe un sous- $\mathcal{O}_{\lambda}$-module $H_{\infty}$ totalement isotrope de $T_{\lambda}(A)$ tel que, si, pour tout entier $n \geq 1, \operatorname{pr}_{n}: T_{\lambda}(A) \rightarrow T_{\lambda}(A) / \ell^{n} T_{\lambda}(A)=T_{\lambda}\left[\lambda^{n}\right]$ désigne la projection canonique, on a

$$
\operatorname{pr}_{r_{H}}\left(H_{\infty}\right)=H^{\prime}
$$

Par le lemme 4.2, on peut supposer que dans une décomposition $T_{\lambda}(A)=H_{\infty} \oplus H_{\infty}^{\prime}$ la forme symplectique s'écrit comme la forme canonique $J$. Pour tout $n \geq 1$, notons

$$
H_{n}:=\operatorname{pr}_{n}\left(H_{\infty}\right)=H_{\infty} / H_{\infty} \cap \ell^{n} T_{\lambda}(A) .
$$

On a pour tout $n \geq 1,[\ell] H_{n+1}=H_{n}$. On peut donc poser

$$
H^{\infty}=\bigcup_{n \geq 1} H_{n} \subset T_{\lambda}\left[\lambda^{\infty}\right]
$$

De plus, on voit que, dans $K\left(T_{\lambda}\left[\lambda^{\infty}\right]\right)$, le groupe de Galois correspondant à $H_{\infty}$ est le même que celui correspondant à $H^{\infty}$. On a

$$
H \subset\left[\ell^{m_{1}(H)}\right]^{-1}\left(H^{\prime}\right)=\left[\ell^{m_{1}(H)}\right]^{-1}\left(H_{r_{H}}\right) \subset\left[\ell^{m_{1}(H)}\right]^{-1}\left(H^{\infty}\right) .
$$

En considérant la multiplication par $\left[\ell^{m_{1}(H)}\right]$ sur $H^{\infty}$, on en déduit (car $H^{\infty}$ est $\ell$-divisible) que

$$
H \subset H^{\infty}+\operatorname{ker}\left[\ell^{m_{1}(H)}\right]=: \widetilde{H^{\infty}},
$$

où $\left[\ell^{n}\right]$ est le morphisme de multiplication dans $T_{\lambda}\left[\lambda^{\infty}\right]$. Ainsi comme dans le cas totalement isotrope, on se ramène à une situation où un lemme de groupe permet de 
conclure : le groupe de $\operatorname{Galois} \operatorname{Gal}\left(K\left(T_{\lambda}\left[\lambda^{\infty}\right]\right) / K\left(\widetilde{H^{\infty}}\right)\right)$ n'est autre que (il s'agit d'une égalité $\asymp$ à indice fini près)

$\left\{M \in \operatorname{GSp}_{2 h}\left(\mathcal{O}_{\lambda}\right) \mid \operatorname{mult}(M) \in \mathbb{Z}_{\ell}^{\times}\right.$et $\left.\forall i \leq g, M e_{g+i}=e_{g+i} \bmod \ell^{m_{1}(H)}, M e_{i}=e_{i}\right\}$.

La même preuve que celle du corollaire 2.11 de [Hindry et Ratazzi 2012] donne alors le résultat : le groupe engendré $\operatorname{par} \operatorname{Gal}\left(K\left(T_{\lambda}\left[\lambda^{\infty}\right]\right) / K\left(\widetilde{H^{\infty}}\right)\right)$ et $\operatorname{Sp}_{2 h}\left(\mathcal{O}_{\lambda}\right)$ est (avec une égalité $\asymp$ à indice fini près)

$$
\left\{M \in \operatorname{GSp}_{2 h}\left(\mathcal{O}_{\lambda}\right) \mid \operatorname{mult}(M) \in \mathbb{Z}_{\ell}^{\times} \text {et } \operatorname{mult}(M) \equiv 1 \bmod \ell^{m_{1}(H)}\right\} .
$$

Notamment, on a,

$$
K(H) \cap K\left(\mu_{\ell^{\infty}}\right) \subset K\left(\widetilde{H^{\infty}}\right) \cap K\left(\mu_{\ell^{\infty}}\right) \subset K\left(\mu_{\ell^{m_{1}(H)}}\right),
$$

la seconde inclusion étant aussi une égalité à indice fini près, i.e.,

$$
K\left(\widetilde{H^{\infty}}\right) \cap K\left(\mu_{\ell^{\infty}}\right) \asymp K\left(\mu_{\ell^{m_{1}(H)}}\right) .
$$

La proposition 5.4 permet de conclure.

\section{Preuve du théorème principal pour $H \subset A[\ell]$}

Soit $A / K$ une variété abélienne sur un corps de nombres, telle que $\operatorname{End}_{K}(A)=$ $\operatorname{End}_{\bar{K}}(A)$. On commence par se ramener au cas $\ell$-adique (cf. [Hindry et Ratazzi 2010, proposition 4.1]) grâce à la presque indépendance rappelée à la proposition 3.2 :

Proposition 6.1. Soit $\alpha>0$. Pour démontrer que $\gamma(A) \leq \alpha$, il suffit de montrer que : il existe une constante strictement positive $C(A / K)$ ne dépendant que de $A / K$ telle que pour tout nombre premier $\ell$, pour tout sous-groupe fini $H_{\ell}$ de $A\left[\ell^{\infty}\right]$, on a

$$
\operatorname{Card}\left(H_{\ell}\right) \leq C(A / K)\left[K\left(H_{\ell}\right): K\right]^{\alpha} .
$$

Remarque 6.2. Rappelons que l'on a supposé que la variété abélienne $A / K$ est telle que $\operatorname{End}_{K}(A)=\operatorname{End}_{\bar{K}}(A)$. Concernant notre question de borne sur la torsion, ceci nous permet de supposer que le groupe fini $H \subset A\left[\ell^{n}\right]$ est en fait un $\operatorname{End}_{\bar{K}}(A)$ module. En effet : notons $H_{E}$ le $\operatorname{End}_{\bar{K}}(A)$-module engendré par $H$ et supposons que l'on ait pour $H_{E}$ une inégalité de la forme suivante, uniformément en $(\ell, H)$,

$$
\left|H_{E}\right| \ll\left[K\left(H_{E}\right): K\right]^{\alpha} .
$$

On a donc $|H| \ll\left[K\left(H_{E}\right): K\right]^{\alpha}$ car $H$ est inclus dans $H_{E}$. Mais $\operatorname{End}_{K}(A)=$ $\operatorname{End}_{\bar{K}}(A)$, donc si $x \in H$ et $f \in \operatorname{End}_{\bar{K}}(A)$ alors $f(x)$ est encore un point de $A$ qui est $K(H)$ rationnel, donc $K(H)=K\left(H_{E}\right)$. En particulier ceci implique que $|H| \ll[K(H): K]^{\alpha}$ comme annoncé. 
Nous nous plaçons dans toute la suite de ce paragraphe dans la situation particulière d'une variété abélienne $A$ définie sur $K$, géométriquement simple de type I ou II, qui est pleinement de type Lefschetz. Nous supposerons de plus que $\ell \notin S_{\mathrm{ex}}(A)$ de sorte à pouvoir appliquer les techniques développées dans les paragraphes précédents. Enfin nous prenons le cas particulier d'une situation horizontale d'un

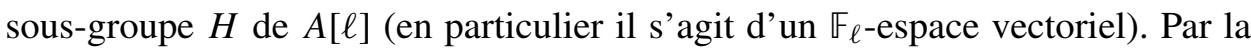
remarque précédente, nous pouvons même supposer que $H$ est un $\mathcal{O}_{E} / \ell \mathcal{O}_{E}$-module. Nous avons la décomposition suivante :

$$
H= \begin{cases}\prod_{\lambda \mid \ell} H[\lambda] \subset \prod_{\lambda \mid \ell} T_{\lambda}[\lambda] & \text { (Type I), } \\ \prod_{\lambda \mid \ell} H[\lambda] \oplus H[\lambda] \subset \prod_{\lambda \mid \ell} T_{\lambda}[\lambda] \oplus T_{\lambda}[\lambda] & \text { (Type II). }\end{cases}
$$

On sait par la proposition 5.2 que pour tout $\ell$ on a,

$$
\rho_{\lambda}\left(G_{K}\right) \stackrel{\circ}{=}\left\{M \in \mathrm{GSp}_{2 h}\left(\mathbb{F}_{\lambda}\right) \mid \operatorname{mult}(M) \in \mathbb{F}_{\ell}^{\times}\right\} .
$$

Dans notre situation les $H[\lambda] \subset T_{\lambda}[\lambda]$ sont des $\mathbb{F}_{\lambda}$-espaces vectoriels. Rappelons que l'on a, uniformément en $(\ell, H)$, l'égalité à indice fini près $\delta(H[\lambda]) \asymp$ $\left[K(H[\lambda]) \cap K\left(\mu_{\ell}\right): K\right]$. On obtient ainsi :

Lemme 6.3. Si $H[\lambda]$ est inclus dans un sous-espace totalement isotrope $d u \mathbb{F}_{\lambda}$ espace vectoriel $T_{\lambda}[\lambda]$ alors, uniformément en $(\ell, H)$, on a $\delta(H[\lambda]) \asymp 1$. Sinon $\delta(H[\lambda]) \asymp \ell$.

Lemme 6.4. Uniformément en $(\ell, H)$, on a

$$
\delta(H[\lambda]) \asymp\left(\mathbb{F}_{\ell}^{\times}: \operatorname{mult}\left(G_{0}(H[\lambda])\right)\right), \quad \text { où } G_{0}(H[\lambda])=\operatorname{Gal}\left(K\left(T_{\lambda}[\lambda]\right) / K(H[\lambda])\right) .
$$

On a de plus

$$
[K(H[\lambda]): K] \stackrel{\circ}{=}\left(\rho_{\lambda}\left(G_{K}\right): G_{0}(H[\lambda])\right) \stackrel{\circ}{=} \delta(H[\lambda])\left(\operatorname{Sp}_{2 h}\left(\mathbb{F}_{\lambda}\right): G(H[\lambda])\right) .
$$

Démonstration. Pour le premier point, on a $\operatorname{Gal}\left(K\left(T_{\lambda}[\lambda]\right) / K\right) \stackrel{\circ}{=} \rho_{\lambda}\left(G_{K}\right)$. Le groupe de $\operatorname{Galois} \operatorname{Gal}\left(K\left(T_{\lambda}[\lambda]\right) / K\left(\mu_{\ell}\right)\right)$ est alors presque égal à

$$
\mathrm{SG}_{\lambda}:=\rho_{\lambda}\left(G_{K}\right) \cap \operatorname{Ker}(\text { mult }) .
$$

Alors $K(H[\lambda]) \cap K\left(\mu_{\ell}\right)$ est la sous-extension fixée par le groupe $U$ engendré par $\mathrm{SG}_{\lambda}$ et $G_{0}(H[\lambda])$. On voit immédiatement que le noyau de $\rho_{\lambda}\left(G_{K}\right) \stackrel{\text { mult }}{\longrightarrow} \mathbb{F}_{\ell}^{\times} \rightarrow$ $\mathbb{F}_{\ell}^{\times} / \operatorname{mult}\left(G_{0}(H[\lambda])\right)$ est le groupe $U$. Pour le second point : la première égalité est donnée par la théorie de Galois car on a que $\operatorname{Gal}\left(K\left(T_{\lambda}[\lambda]\right) / K\right) \stackrel{\circ}{=} \rho_{\lambda}\left(G_{K}\right)$. La seconde égalité est une chasse au diagramme facile.

Notons $d_{\lambda}$ la dimension de $H[\lambda]$ sur $\mathbb{F}_{\lambda}$ et $\left(e_{1}, \ldots e_{d_{\lambda}}\right)$ une base que l'on complète en une base $\left(e_{1}, \ldots, e_{2 h}\right)$ de $T_{\lambda}[\lambda]$. On définit

$$
G(H[\lambda])=\left\{M \in \operatorname{Sp}_{2 h}\left(\mathbb{F}_{\lambda}\right) \mid M e_{i}=e_{i}, \quad 1 \leq i \leq d_{\lambda}\right\}
$$


Notons $\left(\hat{e}_{1}, \ldots, \hat{e}_{2 h}\right)$ une base de $T_{\lambda}(A)$ relevant la base sur $\mathbb{F}_{\lambda}$. Introduisons maintenant le groupe algébrique sur $\mathcal{O}_{\lambda}$ suivant :

$$
G_{1}:=\left\{M \in \mathrm{Sp}_{2 h} \mid M \hat{e}_{i}=\hat{e}_{i}, 1 \leq i \leq d_{\lambda}\right\}
$$

On voit que

$$
G(H[\lambda])=\left\{M \in \operatorname{Sp}_{2 h}\left(\mathbb{F}_{\lambda}\right) \mid M \in G_{1} \bmod \lambda\right\} .
$$

Par changement de base symplectique sur $\mathbb{F}_{\lambda}, G_{1}$ est conjugué sur $\mathbb{F}_{\lambda}$ à l'un des groupes $P_{r, s}$ introduits précédemment. En posant $G=\mathrm{Sp}_{2 h}$, et en rappelant que $\operatorname{Card}\left(\mathbb{F}_{\lambda}\right)=\ell^{f(\lambda)}$, on voit que, d'après lemme 2.3 on a

$$
[K(H[\lambda]): K] \asymp \ell^{m(H[\lambda])} \ell^{f(\lambda) \operatorname{codim} P_{r_{\lambda}, s_{\lambda}},}
$$

où $\left(r_{\lambda}, s_{\lambda}\right)$ (avec éventuellement $s_{\lambda}=0$ ) est le couple correspondant à $H[\lambda]$.

Utilisant le lemme 6.3, le théorème 6.6 de [Hindry et Ratazzi 2010] s'adapte immédiatement (cf. la proposition 7.3 ci-après) pour donner :

Proposition 6.5. Avec les notations précédentes, uniformément en $(\ell, H)$, on a

$$
\ell^{m(H)} \asymp\left[K(H) \cap K\left(\mu_{\ell}\right): K\right] \asymp \max _{\lambda \mid \ell} \ell^{m(H[\lambda])}
$$

et

$$
\left[K(H): K\left(\mu_{\ell^{m(H)}}\right)\right] \asymp \prod_{\lambda \mid \ell}\left[K(H[\lambda]): K\left(\mu_{\left.\left.\ell^{m(H[\lambda])}\right)\right] .}\right.\right.
$$

Cas totalement décomposé. Nous supposons ici que $\ell \notin S_{\mathrm{ex}}(A)$ est totalement décomposé dans $\mathcal{O}_{E}$. Notre situation est alors la suivante :

$$
H=\left\{\begin{array}{clll}
\prod_{\lambda \mid \ell} H[\lambda] \subset \prod_{\lambda \mid \ell} T_{\lambda}[\lambda] & \text { et } & \rho_{\ell}=\prod_{\lambda \mid \ell} \rho_{\lambda} & \text { (Type I), } \\
\prod_{\lambda \mid \ell} H[\lambda] \oplus H[\lambda] \subset \prod_{\lambda \mid \ell} T_{\lambda}[\lambda] \oplus T_{\lambda}[\lambda] & \text { et } & \rho_{\ell}=\prod_{\lambda \mid \ell} \rho_{\lambda} \oplus \rho_{\lambda} & \text { (Type II). }
\end{array}\right.
$$

De plus

$$
\operatorname{Gal}\left(K(A[\ell]) / K\left(\mu_{\ell}\right)\right)=\prod_{\lambda \mid \ell} \operatorname{Gal}\left(K\left(T_{\lambda}[\lambda]\right) / K\left(\mu_{\ell}\right)\right) .
$$

Du point du vue combinatoire, les formules sont identiques à celles d'un produit de variétés abéliennes de type $\mathrm{GSp}_{2 h}$ et, les résultats du paragraphe précédent nous indiquent que la combinatoire n'est finalement autre que celle d'un produit de $e$ variétés abéliennes de type $\mathrm{GSp}_{2 h}$, deux à deux non isogènes. Nous pouvons donc directement en déduire la valeur de l'exposant $\gamma(A)$.

Définition 6.6. Nous noterons dans la suite : $d=1$ si $A$ et de type I et $d=2$ si A est de type II.

Les calculs de [Hindry et Ratazzi 2012, paragraphes 4.1 et 6.2] donnent dans ce cas :

$$
\gamma(A)=\sup _{I \subset\{1, \ldots, e\}} \frac{2 \sum_{\lambda \in I} d h}{1+\left(2 h^{2}+h\right)|I|} .
$$


Ce sup se calcule aisément (le max est atteint pour $I=\{1, \ldots, e\}$ ) et on trouve donc

$$
\gamma(A)=\frac{2 d h e}{1+\left(2 h^{2}+h\right) e}=\frac{2 \operatorname{dim} A}{1+\operatorname{dim} \operatorname{Res}_{E / \mathbb{Q}} \operatorname{Sp}_{2 h}}=\frac{2 \operatorname{dim} A}{\operatorname{dim} \mathrm{MT}(A)} .
$$

Cas général. Nous ne supposons plus désormais que $\ell$ est totalement décomposé, la combinatoire qui résulte est donc différente et il faut dans ce cadre général la refaire explicitement (ceci contient d'ailleurs le cas du sous-paragraphe précédent). On a

$$
H[\lambda]=\left(\mathcal{O}_{\lambda} / \lambda\right)^{r_{\lambda}+s_{\lambda}} \quad \text { avec } s_{\lambda}=0 \Longleftrightarrow H[\lambda] \text { est inclus dans un Lagrangien. }
$$

De plus on a, quitte à réordonner,

$$
\begin{gathered}
0 \leq s_{\lambda} \leq r_{\lambda} \leq h, \quad \text { où } 2 h=\operatorname{dim}_{\mathbb{F}_{\lambda}} T_{\lambda}[\lambda], \\
\sum_{\lambda \mid \ell} f(\lambda)=[E: \mathbb{Q}]=e \quad \text { et } \quad d h e=g=\operatorname{dim} A,
\end{gathered}
$$

où l'on note comme précédemment $d=1$ si $A$ est de type I et $d=2$ si $A$ est de type II.

On obtient finalement, sous les conditions précédentes, la valeur suivante pour le cardinal de $H$ :

$$
\operatorname{Card}(H)=\ell^{d \sum_{\lambda \mid \ell} f(\lambda)\left(r_{\lambda}+s_{\lambda}\right)} .
$$

Le degré de l'extension $[K(H): K]$ dépend selon que les $H[\lambda]$ sont ou non inclus dans des Lagrangiens. Si l'un des $H[\lambda]$ n'est pas inclus dans un Lagrangien alors nous obtenons

$$
[K(H): K] \asymp \ell^{1+\sum_{\lambda \mid \ell} f(\lambda) \operatorname{codim} P_{r_{\lambda}, s_{\lambda}}} .
$$

Si par contre tout les $H[\lambda]$ sont inclus dans un Lagrangien alors nous obtenons

$$
[K(H): K] \asymp \ell^{\sum_{\lambda \mid \ell} f(\lambda) \operatorname{codim} P_{r_{\lambda}, 0}} .
$$

Il reste à interpréter le quotient de l'exposant de $\ell$ du $\operatorname{Card}(\mathrm{H})$ par celui de $[K(H): K]$ pour conclure : c'est l'objet du paragraphe combinatoire suivant.

Combinatoire. Comme précédemment on note $d=1$ si $A$ est de type I et $d=2$ si $A$ est de type II. Nous sommes ramenés à calculer la quantité :

$$
\frac{1}{d} \gamma:=\max _{r_{\lambda}, s_{\lambda}} \frac{\sum_{\lambda \mid \ell} f(\lambda)\left(r_{\lambda}+s_{\lambda}\right)}{\delta+\sum_{\lambda \mid \ell} f(\lambda) \operatorname{codim} P_{r_{\lambda}, s_{\lambda}}}
$$

où le maximum est pris pour $0 \leq s_{\lambda} \leq r_{\lambda} \leq h$ et $\delta$ vaut 0 (resp. 1) si tous les $s_{\lambda}$ sont nuls (resp. si l'un des $s_{\lambda}$ est non nul). 
Proposition 6.7. Soit $\gamma=\gamma(A)$ défini ci-dessus; alors

$$
\gamma=\frac{2 d h e}{1+2 e h^{2}+h e}=\frac{2 \operatorname{dim} A}{\operatorname{dim} \operatorname{MT}(A)} .
$$

Nous donnons ci-dessous, dans le cas particulier de la proposition ci-dessus, une preuve via les interpolateurs de Lagrange. Un argument combinatoire différent, sera donné plus loin dans le cas général de la preuve du lemme 7.5, l'argument suivant n'est donc pas indispensable mais a l'avantage d'être assez direct.

Remarques. (1) On peut réécrire, pour $P_{r, s} \subset \mathrm{Sp}_{2 g}$ :

$$
\operatorname{codim} P_{r, s}=2 g(r+s)-r s-\left(\frac{r^{2}+s^{2}}{2}\right)+\frac{r+s}{2}=\left(2 g+\frac{1}{2}-\frac{r+s}{2}\right)(r+s) .
$$

On observe en particulier que la dimension ou codimension de $P_{r, s}$ ne dépend que de $r+s$.

(2) Nous allons devoir étudier le sens de variation d'une fraction rationnelle de la forme

$$
f(x)=\frac{a-x}{A-x(x-1) / 2}
$$

dont la dérivée s'écrit

$$
f^{\prime}(x)=-2 \frac{(x-a)^{2}+2 A+a-a^{2}}{(2 A-x(x-1))^{2}}
$$

et est donc décroissante dès que $2 A+a \geq a^{2}$.

Démonstration de la proposition 6.7. La preuve consiste à appliquer le calcul différentiel à la fonction de variables $(\underline{r}, \underline{s}):=\left(r_{\lambda}, s_{\lambda}\right)_{\lambda \mid \ell}$ dont on veut évaluer le maximum :

$$
\psi(\underline{r}, \underline{s}):=\frac{N(\underline{r}, \underline{s})}{D(\underline{r}, \underline{s})}:=\frac{\sum_{\lambda \mid \ell} f(\lambda)\left(r_{\lambda}+s_{\lambda}\right)}{\delta+\sum_{\lambda \mid \ell} f(\lambda) \operatorname{codim} P_{r_{\lambda}, s_{\lambda}}}
$$

(nous écrivons la fonction sous la forme "Numérateur/Dénominateur= N/D"). Commençons par traiter le cas où tous les $s_{\lambda}$ sont nuls. Les différentielles des deux fonctions $N$ et $D$ s'écrivent

$$
\partial N=(f(\lambda))_{\lambda \mid \ell} \quad \text { et } \quad \partial D=\left(f(\lambda)\left(2 h-r_{\lambda}+\frac{1}{2}\right)\right)_{\lambda \mid \ell} .
$$

Le théorème de Lagrange indique que, en un maximum de $N / D$, ces deux différentielles sont proportionnelles, donc $2 h-r_{\lambda}+\frac{1}{2}$ est constant, ou encore, $r_{\lambda}=2 h-\kappa$ (avec $h \leq \kappa \leq 2 h$ ). On obtient alors

$$
\frac{N}{D}=\frac{2 h e-\kappa e}{2 \sum_{\lambda \mid \ell} f(\lambda) h^{2}+h e-e \kappa(\kappa-1) / 2}=\frac{2 h-\kappa}{2 h^{2}+h-\kappa(\kappa-1) / 2} .
$$


La fonction à droite est décroissante avec $\kappa$ et donc majorée par la valeur en $\kappa=h$, c'est-à-dire $2 /(3 h+3)$ (noter que $\kappa \geq h$ ).

On traite ensuite le cas général (avec l'un des $s_{\lambda}$ non nul), on pose donc

$$
N:=\sum_{\lambda \mid \ell} f(\lambda)\left(r_{\lambda}+s_{\lambda}\right), \quad D:=1+\sum_{\lambda \mid \ell} f(\lambda)\left(r_{\lambda}+s_{\lambda}\right)\left(2 h+\frac{1}{2}-\frac{r_{\lambda}+s_{\lambda}}{2}\right) .
$$

Le théorème de Lagrange indique maintenant que, en un maximum de $N / D$, on aura $s_{\lambda}+r_{\lambda}=2 h-\kappa$, avec maintenant $0 \leq \kappa \leq 2 h$. En reportant on obtient :

$$
\frac{N}{D} \leq \frac{e(2 h-\kappa)}{1+2 \sum_{\lambda \mid \ell} f(\lambda) h^{2}+e h-e \kappa(\kappa-1) / 2}=\frac{2 h-\kappa}{1 / e+2 h^{2}+h-\kappa(\kappa-1) / 2} .
$$

Cette dernière fonction est décroissante en $\kappa$ donc majorée par la valeur en $\kappa=0$, ce qui donne au final :

$$
\psi \leq \max \left\{\frac{2}{3(h+1)}, \frac{2 h e}{1+2 e h^{2}+e h}\right\}=\frac{2 h e}{1+2 e h^{2}+e h} .
$$

Observons que $\kappa=0$ correspond à $r_{\lambda}+s_{\lambda}=2 h$ donc à $r_{\lambda}=s_{\lambda}=h$. En considérant donc le cas $r_{\lambda}=s_{\lambda}=h$, on obtient

$$
\psi=\frac{2 h e}{1+2 h^{2} e+h e} .
$$

\section{Cas d'un groupe $H$ quelconque}

Dans ce paragraphe nous allons donner une preuve du résultat principal (théorème 1.14). Rappelons que l'on a supposé que la variété abélienne $A / K$ est un produit $\prod_{i=1}^{d} A_{i}^{n_{i}}$ de variétés abéliennes simples, chacune de type I ou II et chacune pleinement de type Lefschetz. Nous avons déjà indiqué que l'on peut supposer de plus que pour tout $i$, les $A_{i}$ sont telles que $\operatorname{End}_{\bar{K}}\left(A_{i}\right)=\operatorname{End}_{K}\left(A_{i}\right)$ et telles que $\operatorname{End}_{K}\left(A_{i}\right)$ est un ordre maximal dans $\operatorname{End}_{K}\left(A_{i}\right) \otimes_{\mathbb{Z}} \mathbb{Q}$.

Définition 7.1. Avec la notation de la définition 3.4, nous noterons dans la suite $S_{\mathrm{ex}}=\bigcup_{i=1}^{d} S_{\mathrm{ex}}\left(A_{i}\right)$.

Dans la suite de ce paragraphe nous supposerons que $\ell \notin S_{\mathrm{ex}}$.

Soit $H$ un sous-groupe fini de $A\left[\ell^{\infty}\right]$. Par le paragraphe 4.2 de [Hindry et Ratazzi 2010], on peut supposer que $H$ s'écrit sous la forme $H=\prod_{i=1}^{d} H_{i}^{n_{i}}$. De plus, par la remarque 6.2, nous pouvons supposer que chaque $H_{i}$ est un $\mathcal{O}_{\ell, i}$-module, inclus dans un $A_{i}\left[\ell^{n}\right]$ pour $n$ convenable (où l'on note $\mathcal{O}_{\ell, i}$ le tensorisé par $\mathbb{Z}_{\ell}$ de $\operatorname{End}\left(A_{i}\right)$ ). Notons

$\mathfrak{I}_{\ell}:=\{(\lambda, i) \mid i \in\{1, \ldots, d\}$,

et $\lambda$ une place du centre de $\operatorname{End}\left(A_{i}\right) \otimes \mathbb{Q}$ au-dessus de $\left.\ell\right\}$. 
Pour $(\lambda, i) \in \mathfrak{I}_{\ell}$, posons $\mathcal{O}_{\lambda, i}$ la composante $\lambda$-adique de $\mathcal{O}_{\ell, i}$ et posons $X_{\lambda, i}$ le morceau de $H_{i}$ correspondant à $\lambda$. Dans le cas de type II, $X_{\lambda, i}$ se décompose à son tour en deux copies isomorphes : $X_{\lambda, i}=H_{\lambda, i} \oplus H_{\lambda, i}$. Dans le cas de type I, on pose $H_{\lambda, i}:=X_{\lambda, i}$. Avec des notations évidentes, les $H_{\lambda, i}$ sont des $\mathcal{O}_{\lambda, i} / \ell^{n} \mathcal{O}_{\lambda, i^{-}}$ sous-modules de $T_{\lambda, i}\left[\lambda^{n}\right]$. On a finalement la décomposition suivante de $H$ :

$$
H=\prod_{(\lambda, i) \in \mathfrak{I}_{\ell}} X_{\lambda, i}^{n_{i}}
$$

En tant que groupe on sait que pour $(\lambda, i) \in \mathfrak{I}_{\ell}$,

$$
\mathcal{O}_{\lambda, i} / \ell^{n} \mathcal{O}_{\lambda, i}=\left(\mathbb{Z} / \ell^{n} \mathbb{Z}\right)^{f(\lambda)} \quad \text { et } \quad T_{\lambda, i}\left[\lambda^{n}\right]=\left(\mathbb{Z} / \ell^{n} \mathbb{Z}\right)^{2 h_{i} f(\lambda)} .
$$

On sait également que, uniformément en $\ell$ et $\lambda$, on a,

$$
\rho_{A_{i}, \lambda \infty}\left(G_{K}\right) \stackrel{\circ}{=}\left\{M \in \operatorname{GSp}_{2 h_{i}}\left(\mathcal{O}_{\lambda, i}\right) \mid \operatorname{mult}(M) \in \mathbb{Z}_{\ell}^{\times}\right\} .
$$

Dans tous les cas on obtient ainsi une égalité à indice fini près, en réduisant modulo $\ell^{n}$.

Soit donc $H_{\lambda, i}$ un sous-groupe fini de $T_{\lambda, i}\left[\lambda^{\infty}\right]$. On pose

$$
G_{0}\left(H_{\lambda, i}\right):=\left\{M \in \mathrm{GSp}_{2 h_{i}}\left(\mathcal{O}_{\lambda, i}\right) \mid \operatorname{mult}(M) \in \mathbb{Z}_{\ell}^{\times}, \text {et } \forall x \in H_{\lambda, i}, M x=x\right\},
$$

et $G\left(H_{\lambda, i}\right):=G_{0}\left(H_{\lambda, i}\right) \cap \operatorname{Sp}_{2 h_{i}}\left(\mathcal{O}_{\lambda, i}\right)$. Comme $\mathcal{O}_{\lambda, i} / \ell^{n} \mathcal{O}_{\lambda, i}$-module et comme groupe abstrait, $H_{\lambda, i}$ est de la forme

$$
H_{\lambda, i} \simeq \prod_{j=1}^{2 h_{i}} \mathcal{O}_{\lambda, i} / \ell^{m_{j}} \mathcal{O}_{\lambda, i} \simeq \prod_{j=1}^{2 h_{i}}\left(\mathbb{Z} / \ell^{m_{j}} \mathbb{Z}\right)^{f(\lambda)},
$$

où nous sous-entendons, pour ne pas alourdir plus que de raison les notations, que les nombres $m_{j}$ dépendent également de $(\lambda, i)$.

Notons $e_{(\lambda, i)}^{1}, \ldots, e_{(\lambda, i)}^{2 h_{i}}$ un système de générateurs (en tant que $\mathcal{O}_{\lambda, i} / \ell^{n} \mathcal{O}_{\lambda, i^{-}}$ module); les $e_{(\lambda, i)}^{j}$ étant d'ordre respectifs $\ell^{m_{j}}$. Notons de plus $\left\{\hat{e}_{(\lambda, i)}^{1}, \ldots, \hat{e}_{(\lambda, i)}^{2 h_{i}}\right\}$ une base du $\mathcal{O}_{\lambda, i}$-module libre $T_{\lambda, i}:=T_{\lambda}\left(A_{i}\right)$ relevant la famille $\left\{e_{(\lambda, i)}^{j,}\right\}$, i.e., telle que $e_{(\lambda, i)}^{j}=\hat{e}_{(\lambda, i)}^{j} \bmod \ell^{m_{j}}$ pour tout $j$. On a

$$
G\left(H_{\lambda, i}\right)=\left\{M \in \operatorname{Sp}_{2 h_{i}}\left(\mathcal{O}_{\lambda, i}\right) \mid M \hat{e}_{(\lambda, i)}^{j}=\hat{e}_{(\lambda, i)}^{j} \bmod \ell^{m_{j}}, 1 \leq j \leq 2 h_{i}\right\} .
$$

Lemme 7.2. Notons $\delta\left(H_{\lambda, i}\right):=\left(\mathbb{Z}_{\ell}^{\times}: \operatorname{mult}\left(G_{0}\left(H_{\lambda, i}\right)\right)\right)$. Uniformément en $(\ell, H)$, on a alors

$$
\left[K\left(H_{\lambda, i}\right): K\right] \asymp\left(\rho_{A_{i}, \lambda}\left(G_{K}\right): G_{0}\left(H_{\lambda, i}\right)\right) \asymp \delta\left(H_{\lambda, i}\right)\left(\operatorname{Sp}_{2 h_{i}}\left(\mathcal{O}_{\lambda, i}\right): G\left(H_{\lambda, i}\right)\right) .
$$

Démonstration. Comme le lemme 6.4. 
Quitte à renuméroter on peut supposer que les exposants $m_{j}$ (correspondants aux $\left.e_{(\lambda, i)}^{j}\right)$ sont ordonnés dans l'ordre décroissant : $m_{1} \geq \cdots \geq m_{2 h_{i}}$. On pose alors

$$
m^{1}:=\max \left\{m_{i} \mid m_{i} \neq 0\right\} \quad \text { et par récurrence } \quad m^{r+1}=\max \left\{m_{i} \mid m_{i}<m^{r}\right\} .
$$

On obtient ainsi une suite strictement décroissante $m^{1}>\cdots>m^{t_{\lambda, i}} \geq 1$ (avec $\left.t_{\lambda, i} \leq 2 h_{i}\right)$. Le groupe $H_{\lambda, i}$ est isomorphe à $\prod_{j=1}^{t_{\lambda, i}}\left(\mathbb{Z} / \ell^{m^{j}} \mathbb{Z}\right){ }^{f(\lambda) a_{j}}$, les $a_{j}$ dépendants de $(\lambda, i)$. On définit ensuite pour tout $1 \leq r \leq t_{\lambda, i}$, les sous-ensembles emboités

$$
I_{r}=\left\{j \in\left\{1, \ldots, 2 h_{i}\right\} \mid m_{j} \geq m^{r}\right\} \quad \text { de cardinal } \quad\left|I_{r}\right|=\sum_{j=1}^{r} a_{j} .
$$

Introduisons maintenant la suite croissante de groupes algébriques sur $\mathcal{O}_{\lambda, i}$ suivants :

$$
\forall 1 \leq r \leq t_{\lambda, i}, \quad G_{r,(\lambda, i)}:=\left\{M \in \operatorname{Sp}_{2 h_{i}} \mid M \hat{e}_{(\lambda, i)}^{j}=\hat{e}_{(\lambda, i)}^{j} \forall j \in I_{t_{\lambda, i}+1-r}\right\} .
$$

On voit que

$$
G\left(H_{\lambda, i}\right)=\left\{M \in \operatorname{Sp}_{2 h_{i}}\left(\mathcal{O}_{\lambda, i}\right) \mid \forall 1 \leq r \leq t_{\lambda, i} \text { on a } M \in G_{r,(\lambda, i)} \bmod \ell^{m^{t_{\lambda, i}+1-r}}\right\} .
$$

Par changement de base symplectique sur $\mathbb{F}_{\lambda}$, le couple $(\lambda, i)$ étant fixé, chacun des $G_{j,(\lambda, i)}$ est conjugué sur $\mathbb{F}_{\lambda}$ à l'un des groupes $P_{r, s}$ introduits au paragraphe 2 . En posant $G=\mathrm{Sp}_{2 h_{i}}$, on voit que, avec les notations du lemme 2.3, on a

$$
G\left(H_{\lambda, i}\right)=H\left(m^{1}, \ldots, m^{t_{\lambda, i}}\right) .
$$

On va donc pouvoir appliquer le lemme 2.3.

Cas d'un morceau $\boldsymbol{H}_{\lambda, i}$. Le couple $(\lambda, i)$ étant fixé nous renoterons dans ce paragraphe $t:=t_{\lambda, i}$ afin de soulager un peu les notations. On peut appliquer le lemme 2.3, uniformément en $(\ell, H)$, on a :

$$
\left(\operatorname{Sp}_{2 h_{i}}\left(\mathcal{O}_{\lambda, i}\right): G\left(H_{\lambda, i}\right)\right) \gg \ell^{\sum_{j=1}^{t} f(\lambda) \operatorname{codim}\left(G_{j,(\lambda, i)}\right)\left(m^{t+1-j}-m^{t+1-(j-1)}\right)},
$$

où l'on a posé $m^{t+1}=0$ et où $\operatorname{codim}\left(G_{j,(\lambda, i)}\right)$ est la codimension de $G_{j,(\lambda, i)}$ dans $\mathrm{Sp}_{2 h_{j}}$. Les groupes algébriques $G_{j,(\lambda, i)}$ étant conjugués sur $\mathbb{F}_{\lambda}$ aux $P_{r, s}$ (avec éventuellement $s=0), \operatorname{codim}\left(G_{j,(\lambda, i)}\right)$ est également la codimension du groupe $P_{r_{j}, s_{j}}$ correspondant. Par ailleurs, la suite des $\left(G_{j,(\lambda, i)}\right)_{j}$ étant croissante, $(\lambda, i)$ étant fixé, la suite des $\left(P_{r_{j}, s_{j}}\right)_{j}$ l'est également. Ceci se traduit par

$$
r_{j} \geq r_{j+1} \quad \text { et } \quad s_{j} \geq s_{j+1} \quad \text { pour tout } j .
$$

Il nous reste à calculer la valeur de $\delta\left(H_{\lambda, i}\right)$ (ou plutôt une minoration de $\delta\left(H_{\lambda, i}\right)$ ). Soit donc $h \in\{0, \ldots, t\}$ maximal tel que $s_{h} \geq 1$ (on pose $h=0$ si $s_{i}=0$ pour tout $i$ ). On a donc

$s_{1} \geq \cdots \geq s_{h}=1>0=s_{h+1}=\cdots=s_{t} \quad$ et $\quad P_{r_{1}, s_{1}} \subset \cdots P_{r_{h}, s_{h}} \subset P_{r_{h+1,0}} \subset \cdots \subset P_{r_{t}}$. 
Posons

$$
\delta_{1}=\cdots=\delta_{h}=1 \quad \text { et } \quad \delta_{h+1}=\cdots=\delta_{t}=0 .
$$

Posons $m^{t+1}=0$. On voit (il s'agit d'une somme télescopique) que

$$
m^{t+1-h}=m^{t+1-h}-m^{t+1}=\sum_{j=1}^{t}\left(m^{t+1-j}-m^{t+1-(j-1)}\right) \delta_{j} .
$$

Or $P_{r_{h}, s_{h}}\left(\right.$ avec $\left.s_{h} \geq 1\right)$ correspond au groupe $G_{h,(\lambda, i)}$ lui même associé à l'ensemble $I_{t+1-h}$. Il correspond donc à un morceau de $H_{\lambda, i}$ sur lequel on voit qu'il existe $P, Q$ d'ordre $\ell^{m^{t+1-h}}$ tel que l'accouplement de Weil de $\ell^{m^{t-h}} P$ et $\ell^{m^{t-h}} Q$ est une racine primitive $\ell$-ème de 1 . Ceci se traduit en disant que

$$
\delta\left(H_{\lambda, i}\right) \geq \phi\left(\ell^{m^{t+1-h}}\right) \asymp \ell^{m^{t+1-h}},
$$

ceci restant valable pour $h=0$. Nous obtenons ainsi la minoration

$$
\left[K\left(H_{\lambda, i}\right): K\right] \gg \ell^{\sum_{j=1}^{t_{\lambda, i}}\left(m^{t_{\lambda, i}+1-j}-m^{t_{\lambda, i}+1-(j-1)}\right)\left(\delta_{j}+f(\lambda) \operatorname{codim} P_{r_{j}, s_{j}}\right)} .
$$

De plus, pour tout entier $k \in\{1, \ldots t\}$,

$$
r_{t+1-k}+s_{t+1-k}=\left|I_{k}\right|=\sum_{j=1}^{k} a_{j} .
$$

Invariant $\boldsymbol{\gamma}(\boldsymbol{A})$ pour $\boldsymbol{H} \subset \boldsymbol{A}\left[\ell^{\infty}\right]$. Nous sommes ici dans la situation présentée au début de cette section avec $H=\prod_{(\lambda, i) \in \mathfrak{I}_{\ell}} X_{\lambda, i}$. Avec les notations introduites dans le cas d'un $H_{\lambda, i}$ (i.e., au paragraphe précédent, page 1874), on peut, pour tout $(\lambda, i) \in \mathfrak{I}_{\ell}$, écrire

$$
H_{\lambda, i}=\prod_{j=1}^{2 h_{i}}\left(\mathbb{Z} / \ell^{m_{j}} \mathbb{Z}\right)^{f(\lambda)}=\prod_{j=1}^{t_{\lambda, i}}\left(\mathbb{Z} / \ell^{m^{j}} \mathbb{Z}\right)^{a_{j} f(\lambda)},
$$

où $\left(m^{j}\right)_{j \geq 1}$ est une suite strictement décroissante (le couple $(\lambda, i)$ étant fixé).

Nous allons utiliser un résultat galoisien que nous avons démontré dans [Hindry et Ratazzi 2010]. Dans le théorème 6.6 de [Hindry et Ratazzi 2010] nous donnons une preuve pour $A=\prod_{i} A_{i}$ et avec $M_{i}=T_{\ell}\left(A_{i}\right)$ (cf. notations ci-dessous). En fait la même preuve reprise mot pour mot donne :

Proposition 7.3. Soient $T_{\ell}(A)=\bigoplus_{j \in J} M_{j}^{\alpha_{j}}$ une décomposition de $\mathbb{Z}_{\ell-\text {-modules ga- }}$ loisiens vérifiant les deux propriétés suivantes (où $M_{j}\left[\ell^{\infty}\right]$ désigne $\bigcup_{n} M_{j} / \ell^{n} M_{j}$ ):

(1) Pour tout $j \in J$ et tout groupe fini $H_{j} \subset M_{j}\left[\ell^{\infty}\right]$, il existe $w_{j}=w_{j}\left(H_{j}\right)$ tels qu'on a, uniformément en $\left(\ell, H_{j}\right)$,

$$
K\left(H_{j}\right) \cap K\left(\mu_{\ell^{\infty}}\right) \asymp K\left(\mu_{\ell^{w_{j}}}\right) .
$$


(2) Uniformément en $\ell$, on a l'identité

$$
\left.\operatorname{Gal}\left(K\left(A\left[\ell^{\infty}\right]\right) / K\left(\mu_{\ell^{\infty}}\right)\right) \asymp \prod_{j \in J} \operatorname{Gal}\left(K\left(M_{j}\left[\ell^{\infty}\right]\right)\right) / K\left(\mu_{\ell^{\infty}}\right)\right) .
$$

Alors si $w:=\max w_{j}$, pour tout groupe fini $H=\prod_{j} H_{j} \subset A\left[\ell^{\infty}\right]$, uniformément en $(\ell, H)$ on $a, K(H) \cap K\left(\mu_{\ell}\right) \asymp K\left(\mu_{\ell^{w}}\right)$ et

$$
\left[K(H): K\left(\mu_{\ell^{w}}\right)\right] \asymp \prod_{j \in J}\left[K\left(H_{j}\right): K\left(\mu_{\ell^{w_{j}}}\right)\right] .
$$

Nous allons appliquer ceci avec l'ensemble $J=\mathfrak{I}_{\ell}$, et pour $j=(\lambda, i) \in \mathfrak{I}_{\ell}$, avec $M_{j}=T_{\lambda, i}\left(A_{i}\right)$, ainsi que $\alpha_{j}=n_{i}$ si $A_{i}$ est de type I et $\alpha_{j}=2 n_{i}$ si $A_{i}$ est de type II. Enfin nous l'utiliserons avec $H_{j}=H_{\lambda, i}$.

Par le lemme 7.2 on a

$$
\left[K\left(H_{\lambda, i}\right): K\right] \asymp \delta\left(H_{\lambda, i}\right)\left(\operatorname{Hdg}\left(A_{i}\right)\left(\mathbb{Z}_{\ell}\right): G\left(H_{\lambda, i}\right)\right) .
$$

Or on sait que dans notre situation on a uniformément en $(\ell, H)$ :

$$
\operatorname{Gal}\left(K\left(A_{i}\left[\ell^{\infty}\right]\right) / K\left(\mu_{\ell^{\infty}}\right)\right) \asymp \prod_{(\lambda, i) \in \mathfrak{I}_{\ell}} \operatorname{Gal}\left(K\left(T_{\lambda, i}\left[\lambda^{\infty}\right]\right) / K\left(\mu_{\ell^{\infty}}\right)\right) .
$$

On peut appliquer la proposition 7.3 et on obtient, uniformément en $(\ell, H)$,

$$
[K(H): K] \asymp \delta(H) \prod_{(\lambda, i) \in \mathfrak{I}_{\ell}}\left(\operatorname{Sp}_{2 h_{i}}\left(\mathcal{O}_{\lambda, i}\right): G\left(H_{\lambda, i}\right)\right) .
$$

Notons $\operatorname{cd}_{(\lambda, i)}^{k}$ la codimension du groupe algébrique $G_{k,(\lambda, i)}$. Dans la situation d'un $H_{\lambda, i}$ fixé nous avions introduit au paragraphe précédent des notations

$$
m_{j} \text { et } a_{j}, 1 \leq j \leq 2 h_{i}, \quad \text { ainsi que } m^{r}, 1 \leq r \leq t_{\lambda, i} .
$$

Afin de rendre claire les diverses dépendances nous utiliserons ci-dessous les notations un peu plus lourdes suivantes en lieu et place des précédentes :

$$
m_{j}(\lambda, i) \text { et } a_{j}(\lambda, i), 1 \leq j \leq 2 h_{i}, \quad \text { ainsi que } \quad m_{\lambda, i}^{r}, 1 \leq r \leq t_{\lambda, i} .
$$

Les calculs effectués dans le cas d'un $H_{\lambda, i}$ nous donnent, uniformément en $(\ell, H)$, $\left(\operatorname{Hdg}(A)\left(\mathbb{Z}_{\ell}\right): G(H)\right)$

$$
\asymp \exp \left(\sum_{(\lambda, i) \in \mathfrak{I}_{\ell}} \sum_{k=1}^{t_{\lambda, i}} f(\lambda) \operatorname{cd}_{(\lambda, i)}^{k}\left(m_{\lambda, i}^{t_{\lambda, i}+1-k}-m_{\lambda, i}^{t_{\lambda, i}+1-(k-1)}\right) \log \ell\right) .
$$

De plus, il existe un $\left(\lambda_{1}, i_{1}\right)$ tel que $\delta(H)=\delta\left(H_{\lambda_{1}, i_{1}}\right)$. Quitte à renuméroter on peut supposer que $i_{1}=1$. On note alors $\left(\delta\left(\lambda_{1}\right)_{j}\right)_{j}$ la suite de 0 et de 1 relative à $\delta\left(H_{\lambda_{1}, 1}\right)$ 
définie au paragraphe précédent. On a, uniformément en $(\ell, H)$,

$$
\delta(H) \gg \exp \left(\sum_{j=1}^{t_{\lambda_{1}, 1}}\left(m_{\lambda_{1}, 1}^{t_{\lambda_{1}, 1}+1-j}-m_{\lambda_{1}, 1}^{t_{\lambda_{1}, 1}+1-(j-1)}\right) \delta\left(\lambda_{1}\right)_{j} \log \ell\right) .
$$

On pose par ailleurs $\delta(\lambda)_{j}=0$ pour tout $j$ si $(\lambda, i) \neq\left(\lambda_{1}, 1\right)$. Avec ces notations, on trouve en suivant les calculs du cas d'un $H_{\lambda, i}$, la minoration suivante (au sens $\gg$, uniformément en $(\ell, H))$ pour $[K(H): K]$ :

$$
\begin{aligned}
\exp \left(\sum _ { ( \lambda , i ) \in \mathfrak { I } _ { \ell } } \sum _ { j = 1 } ^ { t _ { \lambda , i } } m _ { \lambda , i } ^ { j } \left[\left(\delta(\lambda)_{t_{\lambda, i}+1-j}-\right.\right.\right. & \left.\delta(\lambda)_{t_{\lambda, i}+1-(j-1)}\right) \\
& \left.\left.+f(\lambda)\left(\operatorname{cd}_{(\lambda, i)}^{t_{\lambda, i}+1-j}-\operatorname{cd}_{(\lambda, i)}^{t_{\lambda, i}+1-(j-1)}\right)\right] \log \ell\right),
\end{aligned}
$$

et

$$
|H|=\exp \left(\sum_{(\lambda, i) \in \mathfrak{I}_{\ell}} n_{i} d_{i} \sum_{j=1}^{t_{\lambda, i}} m_{\lambda, i}^{j} f(\lambda) a_{j}(\lambda, i) \log \ell\right),
$$

où $d_{i}$ vaut 1 (respectivement 2) si $A_{i}$ est de type I (respectivement de type II) et où l'on rappelle que $A=\prod_{i=1}^{d} A_{i}^{n_{i}}$.

Notons

$$
b_{\lambda, i}^{j}:=\left(\delta(\lambda)_{t_{\lambda, i}+1-j}-\delta(\lambda)_{t_{\lambda, i}+1-(j-1)}\right)+f(\lambda)\left(\operatorname{cd}_{\lambda, i}^{t_{\lambda, i}+1-j}-\operatorname{cd}_{\lambda, i}^{t_{\lambda, i}+1-(j-1)}\right),
$$

et posons de plus

$$
a_{\lambda, i}^{j}:=n_{i} d_{i} a_{j}(\lambda, i)
$$

Avec ces notations, on aura donc, uniformément en $(\ell, H)$, l'inégalité $|H| \ll$ $[K(H): K]^{\gamma}$ si

$$
\gamma \geq \max \frac{\sum_{(\lambda, i) \in \mathfrak{I}_{\ell}} \sum_{j=1}^{t_{\lambda, i}} m_{\lambda, i}^{j} f(\lambda) a_{\lambda, i}^{j}}{\sum_{(\lambda, i) \in \mathfrak{I}_{\ell}} \sum_{j=1}^{t_{\lambda, i}} m_{\lambda, i}^{j} b_{\lambda, i}^{j}}
$$

le max étant pris sur les $m_{\lambda, i}^{1} \geq \cdots \geq m_{\lambda, i}^{t_{\lambda, i}}$ pour $(\lambda, i) \in \mathfrak{I}_{\ell}$.

Ainsi, en invoquant le lemme combinatoire (lemme 2.7) et en suivant les notations et calculs du cas d'un $H_{\lambda, i}$, on voit que l'inégalité $|H| \ll[K(H): K]^{\gamma}$ est vraie uniformément en $(\ell, H)$, si

$$
\gamma \geq \max \frac{\sum_{(\lambda, i) \in \mathfrak{I}_{\ell}} n_{i} d_{i} f(\lambda)\left(r(\lambda, i)_{t_{\lambda, i}+1-h_{\lambda, i}}+s(\lambda, i)_{t_{\lambda, i}+1-h_{\lambda, i}}\right)}{\delta\left(\lambda_{1}\right)_{t_{\lambda_{1}, 1}+1-h_{\lambda_{1}, 1}}+\sum_{(\lambda, i) \in \mathfrak{I}_{\ell}} f(\lambda) \operatorname{codim} P_{r(\lambda, i)_{t_{\lambda, i}+1-h_{\lambda, i}} s(\lambda, i)_{t_{\lambda, i}+1-h_{\lambda, i}}}} .
$$


Ce dernier max se réécrit sous la forme

$$
\max _{\substack{1 \leq r_{\lambda, i} \\ 0 \leq s_{\lambda, i} \leq r_{\lambda, i} \leq h_{i}}} \frac{\sum_{(\lambda, i) \in \mathfrak{I}_{\ell}} n_{i} d_{i} f(\lambda)\left(r_{\lambda, i}+s_{\lambda, i}\right)}{\delta+\sum_{(\lambda, i) \in \mathfrak{I}_{\ell}} f(\lambda)\left(r_{\lambda, i}+s_{\lambda, i}\right)\left(2 h_{i}-\frac{1}{2}\left(r_{\lambda, i}+s_{\lambda, i}-1\right)\right)},
$$

et où en reprenant la définition de $\delta\left(\lambda_{1}\right)_{t_{\lambda_{1}, 1}+1-h_{\lambda_{1}, 1}}$ on voit que $\delta=0$ si tout les $s_{\lambda, i}$ sont nuls et $\delta=1$ si l'un des $s_{\lambda, i}$ est non nul.

Il y a en fait deux évaluations à faire selon que $\delta=1$ ou que $\delta=0$.

1. Si $\delta=1$ alors le max à évaluer se réécrit naturellement sous la forme suivante :

$$
\max _{1 \leq x_{\lambda, i} \leq 2 h_{i}} \frac{\sum_{(\lambda, i) \in \mathfrak{I}_{\ell}} m_{i} d_{i} f(\lambda) x_{\lambda, i}}{1+\sum_{(\lambda, i) \in \mathfrak{I}_{\ell}} f(\lambda) x_{\lambda, i}\left(2 h_{i}-\frac{1}{2}\left(x_{\lambda, i}-1\right)\right)} .
$$

Posons

$$
\rho_{1}(\underline{x}):=\frac{\sum_{(\lambda, i) \in \mathfrak{I}_{\ell}} m_{i} d_{i} f(\lambda) x_{\lambda, i}}{1+\sum_{(\lambda, i) \in \mathfrak{I}_{\ell}} f(\lambda) x_{\lambda, i}\left(2 h_{i}-\frac{1}{2}\left(x_{\lambda, i}-1\right)\right)} .
$$

On rappelle que l'on veut comparer $\rho_{1}(\underline{x})$ avec la quantité

$$
\alpha(A):=\max _{I \subset\{1, \ldots, r\}} \frac{2 \sum_{i \in I} m_{i} g_{i}}{1+\sum_{i \in I} 2 e_{i} h_{i}^{2}+e_{i} h_{i}} .
$$

Lemme 7.4. Pour tout $i \in\{1, \ldots, r\}$, on $a \alpha(A) \geq\left(m_{i} d_{i}\right) /\left(h_{i}+1\right)$.

Démonstration. C'est un calcul immédiat.

Lemme 7.5. On a $\max _{1 \leq x_{\lambda, i} \leq 2 h_{i}} \rho_{1}(\underline{x}) \leq \alpha(A)$.

Démonstration. L'inégalité $\rho_{1}(\underline{x}) \leq \alpha(A)$ se réécrit

$$
\sum_{(\lambda, i) \in \mathfrak{I}_{\ell}} f(\lambda)\left[x_{\lambda, i}^{2}-\left(4 h_{i}+1-\frac{2 m_{i} d_{i}}{\alpha(A)}\right) x_{\lambda, i}\right] \leq 2 .
$$

Par le lemme précédent, on voit dans la somme dans le membre de gauche de l'inégalité, que les indices tels que $x_{\lambda, i} \leq 2 h_{i}-1$ contribuent via un terme négatif à la somme. Autrement dit, la valeur $\rho_{1}(\underline{x})-\alpha(A)$ est maximale quand pour tout les indices on a $x_{\lambda, i}=2 h_{i}$. Mais dans ce cas, en utilisant que

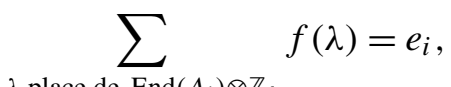


on a

$$
\begin{aligned}
\rho_{1}\left(2 h_{i}, \ldots, 2 h_{i}\right)-\alpha(A) \leq 0 & \Longleftrightarrow \sum_{(\lambda, i) \in \mathfrak{I}_{\ell}} f(\lambda)\left[4 h_{i}^{2}-\left(4 h_{i}+1-\frac{2 m_{i} d_{i}}{\alpha(A)}\right) 2 h_{i}\right] \leq 2 \\
& \Longleftrightarrow \sum_{i=1}^{r}\left(-4 h_{i}^{2}-2 h_{i}+\frac{4 m_{i} h_{i} d_{i}}{\alpha(A)}\right) \sum_{\lambda} f(\lambda) \leq 2 \\
& \Longleftrightarrow \sum_{i=1}^{r}\left(-4 h_{i}^{2} e_{i}-2 h_{i} e_{i}+\frac{4 m_{i} h_{i} e_{i} d_{i}}{\alpha(A)}\right) \leq 2 \\
& \Longleftrightarrow \frac{2 \operatorname{dim} A}{\operatorname{dim} \operatorname{MT}(A)} \leq \alpha(A) .
\end{aligned}
$$

La dernière assertion de la série d'équivalences est vraie, ce qui conclut.

2. Si $\delta=0$ alors dans ce cas un calcul du même type (plus facile) permet également de conclure.

\section{Petites valeurs de $\ell$ exceptionnelles}

Dans ce paragraphe nous indiquons quelles modifications apporter pour les valeurs exceptionnelles de $\ell$ (en nombre fini). On se place dans le cadre d'une variété abélienne géométriquement simple $A / K$ de type I ou II et pleinement de type Lefschetz, telle que $\operatorname{End}_{K}(A)=\operatorname{End}_{\bar{K}}(A)$ et telle que $\operatorname{End}_{K}(A)$ est un ordre maximal de $D:=\operatorname{End}_{K}(A) \otimes_{\mathbb{Z}} \mathbb{Q}$. Nous notons $E$ le centre de $D$ et nous notons enfin $\phi: A \rightarrow A^{\vee}$ une polarisation fixée avec $A$ (les diverses constantes intervenant dépendant de $A$, dépendent aussi du degré de cette polarisation).

Notons que, dans le cas où nous nous sommes placés (pleinement de type Lefschetz), on sait que la conjecture de Mumford-Tate est vraie, donc que l'on a l'inclusion suivante qui est une égalité à indice fini près (dépendant éventuellement de $\ell$ mais peu importe ici car on travaille uniquement avec un nombre fini de valeurs de $\ell)$ :

$$
\rho_{\lambda \infty}\left(G_{K}\right) \subset\left\{x \in \operatorname{GSp}_{2 h}\left(\mathcal{O}_{\lambda}\right) \mid \operatorname{mult}(x) \in \mathbb{Z}_{\ell}^{\times}\right\} .
$$

Nous indiquons dans ce qui suit les petites modifications à faire pour pouvoir traiter les $\ell$ qui sont dans l'ensemble fini exceptionnel $S_{\mathrm{ex}}(A)$ introduit dans la définition 3.4.

Si $\ell$ est ramifié dans $\mathcal{O}_{E}$. On suppose dans ce paragraphe que $\ell$ ne divise pas $\operatorname{deg}(\phi)$ et, dans le cas de type II, que $\ell$ est tel que l'algèbre de quaternions $D$ est décomposée en $\lambda \mid \ell$. On suppose par contre que $\ell$ est ramifié dans $\mathcal{O}_{E}$ :

$$
\ell \mathcal{O}_{E}=\prod_{\lambda \mid \ell} \lambda^{e(\lambda)}
$$


Nous notons toujours $f(\lambda)$ le degré du corps résiduel en la place $\lambda$. Le lemme 3.3 produit l'accouplement $\phi_{\ell^{\infty}}^{\star}$ sur $T_{\ell}(A) \times T_{\ell}(A)$ à valeurs dans $\mathcal{O}_{\ell}^{\star}$.

Hypothèse. Supposons pour l'instant pour simplifier que $\phi_{\ell^{\infty}}^{\star}$ est en fait à valeurs dans $\mathcal{O}_{\ell}$. Nous verrons plus loin comment faire dans le cas général.

Rappelons que dans cette situation on a la décomposition

$$
T_{\ell}(A)= \begin{cases}\prod_{\lambda \mid \ell} T_{\lambda}(A) & \text { (Type I) } \\ \prod_{\lambda \mid \ell} T_{\lambda}(A) \oplus T_{\lambda}(A) & \text { (Type II) }\end{cases}
$$

Par réduction modulo $\lambda^{n}$, on obtient alors pour tout entier $+\infty \geq n \geq 1$, comme dans le cas non ramifié,

$$
\phi_{\lambda^{n}}: T_{\lambda}(A) / \lambda^{n} T_{\lambda}(A) \times T_{\lambda}(A) / \lambda^{n} T_{\lambda}(A) \rightarrow O_{\lambda} / \lambda^{n}(1) .
$$

Notons par ailleurs que $\ell O_{\lambda}=\lambda^{e(\lambda)}$, donc par réduction modulo $\ell^{n}$ on a

et

$$
A\left[\ell^{n}\right]=T_{\ell}(A) / \ell^{n} T_{\ell}(A)= \begin{cases}\prod_{\lambda \mid \ell} T_{\lambda}\left[\lambda^{e(\lambda) n}\right] & \text { (Type I), } \\ \prod_{\lambda \mid \ell} T_{\lambda}\left[\lambda^{e(\lambda) n}\right] \oplus T_{\lambda}\left[\lambda^{e(\lambda) n}\right] & \text { (Type II) }\end{cases}
$$

$$
\phi_{\lambda^{e(\lambda) n}}: T_{\lambda}\left[\lambda^{e(\lambda) n}\right] \times T_{\lambda}\left[\lambda^{e(\lambda) n}\right] \rightarrow \mathcal{O}_{\lambda} / \ell^{n} \mathcal{O}_{\lambda}(1) .
$$

De plus on vérifie que

$$
\phi_{\lambda^{e(\lambda) n}}(\ell x, \ell y)=\phi_{\lambda^{e(\lambda)(n+1)}}(x, y)^{\ell}
$$

et on voit que l'action de Galois sur les accouplements $\phi_{\lambda^{e}(\lambda) n}$ se fait via le caractère cyclotomique $\chi_{\ell^{n}}$. Par ailleurs, le $\mathbb{Z}_{\ell}$-module $\mathcal{O}_{\lambda}$ étant libre de rang $e(\lambda) f(\lambda)$, on a

$$
\begin{gathered}
O_{\lambda} / \ell^{n} \mathcal{O}_{\lambda} \simeq\left(\mathbb{Z} / \ell^{n} \mathbb{Z}\right)^{e(\lambda) f(\lambda)}, \\
T_{\lambda}\left[\lambda^{e(\lambda) n}\right] \simeq\left(O_{\lambda} / \ell^{n} \mathcal{O}_{\lambda}\right)^{2 h} \simeq\left(\mathbb{Z} / \ell^{n} \mathbb{Z}\right)^{2 h e(\lambda) f(\lambda)} .
\end{gathered}
$$

Finalement en travaillant en lieu de place de $\phi_{\lambda^{n}}$ avec $\phi_{\lambda^{e}(\lambda) n}$, en décomposant $H$ selon les $H_{\lambda} \subset T_{\lambda}\left[\lambda^{e(\lambda) n}\right] \subset A\left[\ell^{n}\right]$, on peut reprendre tout ce qui a été fait dans le cas non ramifié. Notamment la propriété $\mu$ pour les $H_{\lambda}$ est toujours vérifiée avec la modification évidente suivante dans la définition de $m_{1}\left(H_{\lambda}\right)$ (et de même pour $\left.m\left(H_{\lambda}\right)\right)$ : on pose

$m_{1}\left(H_{\lambda}\right):=\max \left\{m \mid \exists P, Q \in H_{\lambda}\right.$ d'ordre $\ell^{k}$ tels que $\phi_{\lambda^{e}(\lambda) k}(P, Q)$ est d'ordre $\left.\ell^{m}\right\}$.

Pour prouver la propriété $\mu$ dans ce cadre on peut toujours utiliser le paragraphe sur les modules isotropes sur $\left(\mathcal{O}_{\lambda}, \mathbb{F}_{\lambda}\right)$, puisque les accouplements $\lambda$-adiques $\phi_{\lambda^{r}}$ sont construits modulo $\lambda^{r}$ pour tout entier $r \geq 1$ et non pas uniquement modulo $\ell^{r}$ (cette réduction modulo $\ell^{r}$ étant celle utilisée pour définir les $H_{\lambda}$ ).

En reprenant mot pour mot les calculs combinatoires déjà effectués, on voit avec (4) que les calculs restent inchangés sous réserve de remplacer partout $f(\lambda)$ 
par $f(\lambda) e(\lambda)$. La contrainte $\sum f(\lambda)=[E: \mathbb{Q}]$ étant remplacée par la contrainte $\sum e(\lambda) f(\lambda)=[E: \mathbb{Q}]$, on voit que la valeur $\gamma$ reste la même dans ce cadre, ce que l'on voulait prouver.

Suppression de l'hypothèse. On ne suppose désormais plus que $\phi_{\ell^{\infty}}^{\star}$ est à valeurs dans $\mathcal{O}_{\ell}$.

On sait que $\mathcal{O}_{\lambda}^{\star}$ est un idéal fractionnaire de $E_{\lambda}$, donc de la forme $\pi_{\lambda}^{-m_{\lambda}} \mathcal{O}_{\lambda}$ pour un certain entier $m_{\lambda}$ (avec $\pi_{\lambda}$ une uniformisante). On choisit

$$
m_{0}:=\operatorname{pgcd}\left\{m_{\lambda}|\lambda| \ell, \ell \text { ramifié dans } \mathcal{O}_{E}\right\} .
$$

On a ainsi $\ell^{m_{0}} \mathcal{O}_{\ell}^{\star} \subset \mathcal{O}_{\ell}$ pour tout les $\ell$ que l'on considère. On fait alors les modifications suivantes :

(1) On remplace au départ le module $T_{\ell}(A)$ par $T_{\ell}^{\prime}:=\ell^{m_{0}} T_{\ell}(A)$. Dans ce cas, le lemme 3.3 produit l'acccouplement $\phi_{\ell \infty}^{\star}$ sur $T_{\ell}^{\prime} \times T_{\ell}^{\prime}$ à valeurs dans $\mathcal{O}_{\ell}$ (c'est pour arriver dans $\mathcal{O}_{\ell}$ que l'on a remplacé $T_{\ell}(A)$ par $\left.T_{\ell}^{\prime}\right)$.

(2) On travaille avec $H^{\prime}=\ell^{m_{0}} H$ en lieu et place de $H$. La raison de cette modification est que le groupe $H$ n'est a priori pas contenu dans la réduction modulo $\ell^{n}$ de $T_{\ell}^{\prime}$. Par contre $H^{\prime}$ l'est.

À la déperdition près d'indice en $\ell^{2 h m_{0}}$ près, on peut reprendre la preuve déjà effectuée et on obtient, uniformément en $H$ :

$$
\left|H^{\prime}\right| \ll\left[K\left(H^{\prime}\right): K\right]^{\gamma(A)} \leq[K(H): K]^{\gamma(A)} .
$$

De plus, on a visiblement $|H| \leq\left|H^{\prime}\right| \cdot\left|A\left[\ell^{m_{0}}\right]\right|$. Les premiers problématiques $\ell$ étant en nombre bornés, il en est de même pour le cardinal des divers $A\left[\ell^{m_{0}}\right]$ et on voit donc que l'on obtient ainsi, uniformément en $H$,

$$
|H| \ll[K(H): K]^{\gamma(A)} .
$$

Si $\ell$ divise le degré de la polarisation. On suppose désormais que $\ell$ est un premier quelconque divisant $\operatorname{deg}(\phi)$ et, dans le cas de type II, que $\ell$ est tel que l'algèbre de quaternions $D$ est décomposée en $\lambda \mid \ell$. Notons $m_{0}$ l'entier maximal tel que $\ell^{m_{0}}$ divise $\operatorname{deg}(\phi)$. Dans ce cas, toute les constructions faites jusqu'à présent continuent encore à s'appliquer à condition de faire au départ les modifications suivantes :

(1) On travaille avec $T_{\ell}^{\prime}(A):=\ell^{m_{0}} T_{\ell}(A)$ en lieu et place de $T_{\ell}(A)$.

(2) On travaille avec l'accouplement $\phi_{\ell}^{\phi}: T_{\ell}^{\prime}(A) \times T_{\ell}^{\prime}(A) \rightarrow T_{\ell}^{\prime}(A) \times T_{\ell}^{\prime}\left(A^{\vee}\right) \rightarrow$ $\mathbb{Z}_{\ell}(1)$ défini par $(x, y) \mapsto \phi_{\ell}(x, \phi(y))$, en lieu et place de l'accouplement $\phi_{\ell}$. Ce choix ainsi que le point précédent sont faits de sorte à avoir un accouplement bilinéaire alterné sur $T_{\ell}^{\prime}(A)$, non dégénéré modulo $\ell^{n}$ pour tout $n \geq 1$. 
(3) On travaille avec $H^{\prime}=\ell^{m_{0}} H$ en lieu et place de $H$. La raison de cette modification est que le groupe $H$ n'est a priori pas contenu dans la réduction modulo $\ell^{n}$ de $T_{\ell}^{\prime}(A)$. Par contre $H^{\prime}$ l'est.

Avec ces modifications on peut reprendre la preuve déjà effectuée et on obtient, uniformément en $H$ :

$$
\left|H^{\prime}\right| \ll\left[K\left(H^{\prime}\right): K\right]^{\gamma(A)} \leq[K(H): K]^{\gamma(A)} .
$$

De plus, on a visiblement $|H| \leq\left|H^{\prime}\right|+\left|A\left[\ell^{m_{0}}\right]\right|$. Les premiers $\ell$ étant en nombre fini, le cardinal des divers $A\left[\ell^{m_{0}}\right]$ est donc borné et l'on obtient ainsi uniformément en $H$,

$$
|H| \ll[K(H): K]^{\gamma(A)} .
$$

Petites valeurs exceptionnelles pour le type II. Dans le cas d'une variété abélienne de type II, il y a encore un nombre fini de valeurs $\ell$ exceptionnelles à traiter : les premiers $\ell$ tels que l'algèbre $D$ est non décomposée en $\lambda \mid \ell$. Ce cas des $\ell$ ramifiés dans l'algèbre de quaternions doit être traité avec une légère modification : la décomposition $V_{\ell}(A)=\prod_{\lambda}\left(W_{\lambda}(A) \oplus W_{\lambda}(A)\right)$ n'existant que après tensorisation par une extension quadratique (voir proposition 3.5). Avec les notations du début du section 7 : au lieu d'avoir sur $\mathcal{O}_{\lambda, i}$ la décomposition $X_{\lambda, i}=H_{\lambda, i} \oplus H_{\lambda, i}$, on a, en travaillant sur une extension quadratique de $\mathcal{O}_{\lambda, i}$, la décomposition $X_{\lambda, i}=$ $H_{\lambda, i} \oplus \bar{H}_{\lambda, i}$, où $\bar{H}_{\lambda, i}$ est conjugué à $H_{\lambda, i}$. Ainsi, au lieu de comparer le cardinal de $H_{\lambda, i}$ avec le degré de l'extension [ $K\left(H_{\lambda, i}\right): K$ ], on reprend la même preuve en travaillant directement avec $X_{\lambda, i}$, comparant le cardinal de $X_{\lambda, i}$ et le degré de $\left[K\left(X_{\lambda, i}\right): K\right]$.

\section{Ordre d'un point et degré de l'extension qu'il engendre}

Nous donnons dans ce paragraphe la preuve du théorème 1.12. Nous pouvons pour cela supposer (et nous le faisons) que tous les $\bar{K}$-endomorphismes de $A$ sont définis sur $K$.

Soit $P$ un point de torsion et $H_{P}$ le End $(A)$-module engendré par $P$. On a clairement $K(P)=K\left(H_{P}\right)$. En remarquant que codim $P_{1,0}=2 h\left(\right.$ dans $\left.\mathrm{Sp}_{2 h}\right)$, les arguments des paragraphes 6 et 7 précédents montrent qu'un point $P_{\lambda}$ d'ordre $\ell^{n}$ dans $T_{\lambda}\left[\lambda^{\infty}\right]$ engendre (uniformément en $(\ell, P)$ ) une extension de degré

$$
\left[K\left(P_{\lambda}\right): K\right] \gg \ell^{2 h n} .
$$

Si ensuite $P=\sum_{\lambda \mid \ell} P_{\lambda}$ avec $P_{\lambda}$ point de $T_{\lambda}\left[\lambda^{\infty}\right]$ et d'ordre $\ell^{n_{\lambda}}$, de sorte que $P$ est d'ordre $\ell^{n}$ avec $n=\max n_{\lambda}$, alors, uniformément en $(\ell, P)$ on a

$$
[K(P): K] \gg \ell^{2 h \sum_{\lambda} n_{\lambda}} \geq \ell^{2 h n} .
$$


Enfin si $P$ est d'ordre $m$ quelconque avec $m=\prod_{i=1}^{r} \ell_{i}^{n_{i}}$, on peut écrire $P=\sum_{i=1}^{r} P_{i}$ avec $P_{i}$ d'ordre $\ell_{i}^{n_{i}}$. L'indépendance des représentations $\ell$-adiques (proposition 3.2) permet d'écrire, uniformément en $(\ell, P)$,

$$
[K(P): K]=\left[K\left(P_{1}, \ldots, P_{r}\right): K\right] \gg \prod_{i=1}^{r}\left[K\left(P_{i}\right): K\right] \geq \prod_{i=1}^{r} c_{1} \ell^{2 h n_{i}}=c_{1}^{\omega(m)} m^{2 h} .
$$

\section{Appendice : compléments autour de la conjecture de Mumford-Tate}

Indice de l'image de Galois dans le groupe de Mumford-Tate. La conjecture de Mumford-Tate dit que l'inclusion $G_{\ell^{\infty}}^{0} \subset \mathrm{MT} \otimes \mathbb{Q}_{\ell}$ est une égalité, ou encore que, quitte a avoir effectué une extension finie du corps de base $K$, l'image de la représentation $\ell$-adique galoisienne $\rho\left(G_{K}\right)$ est contenue et ouverte dans $\operatorname{MT}\left(\mathbb{Q}_{\ell}\right)$, ou encore comme $\mathrm{GL}\left(T_{\ell}(A)\right) \cong \mathrm{GL}_{2 g}\left(\mathbb{Z}_{\ell}\right)$ est compact, la conjecture équivaut à dire que, quitte a avoir effectué une extension finie du corps de base $K, \rho\left(G_{K}\right)$ est d'indice fini dans $\mathrm{MT}\left(\mathbb{Z}_{\ell}\right)$. Une forme légèrement plus forte, suggérée par Serre, affirme que cet indice est borné indépendamment de $\ell$. Clarifions tout d'abord ce point en montrant que la conjecture de Mumford-Tate entraîne la forme "forte".

Théorème 10.1. Si A/K vérifie la conjecture de Mumford-Tate alors l'indice de $\rho\left(G_{K}\right)$ dans $\operatorname{MT}(A)\left(\mathbb{Z}_{\ell}\right)$ est borné indépendamment de $\ell$.

La preuve consiste à réunir un résultat de Serre [1986a] (resp. de Wintenberger [2002]) concernant la partie torique centrale (resp. la partie semi-simple) des groupes $\ell$-adiques et des groupes de Mumford-Tate. Plus précisément, notons $S=S_{A}$ le groupe dérivé du groupe de Mumford-Tate de $A$ ou, ce qui revient au même, du groupe de Hodge et notons $C$ la composante neutre du centre du groupe $\operatorname{MT}(A)$; ce sont des $\mathbb{Q}$-groupes algébriques. Notons similairement $S_{\ell}=S_{\ell, A}$ le groupe dérivé de $G_{\ell, A}$ ou, ce qui revient au même, du groupe $H_{\ell, A}$ et notons $C_{\ell}$ la composante neutre du centre du groupe $G_{\ell, A}$; ce sont des $\mathbb{Q}_{\ell}$-groupes algébriques.

On sait par les travaux de Borovoř [1974], Deligne [1982, Exposé I, 2.9, 2.11], et Pjateckiǔ-Šapiro [1971] que

$$
C_{\ell} \subset C_{\mathbb{Q}_{\ell}} \quad \text { et } \quad S_{\ell} \subset S_{\mathbb{Q}_{\ell}} \text {. }
$$

En fait on sait même que la première inclusion est une égalité, essentiellement d'après la théorie abélienne de Serre [1998], une preuve est détaillée dans [Vasiu 2008] et reprise dans [Ullmo et Yafaev 2013]. On ne sait pas, en général si la deuxième inclusion est une égalité, en fait l'égalité est équivalente à la conjecture de Mumford-Tate. D'après Faltings, les deux groupes réductifs ont le même commutant, leur égalité est aussi équivalente à l'égalité des rangs des deux groupes semi-simples. 
Posons $V_{\ell}(A)=T_{\ell}(A) \otimes_{\mathbb{Z}_{\ell}} \mathbb{Q}_{\ell}$. Les groupes $C$ et $S$ sont des sous-groupes du groupe de Mumford-Tate $\mathrm{MT}=\mathrm{MT}_{A}$. En voyant $\mathrm{MT}_{\mathbb{Q}_{\ell}}$ comme un sous-groupe algébrique de $\mathrm{GL}_{V_{\ell}(A)} \cong \mathrm{GL}_{2 g, \mathbb{Q}_{\ell}}$, on peut étendre ces groupes sur $\mathbb{Z}_{\ell}$ en prenant leur adhérence de Zariski dans $\mathrm{GL}_{T_{\ell}(A)}$. Avec un léger abus de notation nous noterons $C\left(\mathbb{Z}_{\ell}\right)$ (resp. $S\left(\mathbb{Z}_{\ell}\right)$ ) le groupe des $\mathbb{Z}_{\ell}$-points de $C$ (resp. $S$ ) vu comme groupe algébrique sur $\mathbb{Z}_{\ell}$. Le même procédé nous permet d'étendre $C_{\ell}$ (resp. $S_{\ell}$ ) en un groupe sur $\mathbb{Z}_{\ell}$.

Concernant la partie torique centrale, nous savons donc que $\rho_{\ell^{\infty}}\left(G_{K}\right) \cap C\left(\mathbb{Z}_{\ell}\right)$ est d'indice fini dans $C\left(\mathbb{Z}_{\ell}\right)$. Le résultat suivant de Serre précise ce point et est un des deux points clef pour la preuve du théorème 10.1.

Proposition 10.2 [Serre 1986a, p. 60]. L'indice $\left(C\left(\mathbb{Z}_{\ell}\right): C_{\ell}\left(\mathbb{Z}_{\ell}\right) \cap \rho_{\ell \infty}\left(G_{K}\right)\right)=: c_{\ell}$ est fini borné indépendamment de $\ell$.

Notons que la preuve de Serre [1986a] est rédigée dans le cas $\operatorname{End}(A)=\mathbb{Z}$ et esquissée dans le cas général. Pour la commodité du lecteur nous donnons ci-dessous une description du centre et de la relation avec l'image de Galois.

Concernant la partie semi-simple $S_{\ell}$ de $G_{\ell}$, suivant Wintenberger [2002], notons $S_{\ell, \mathrm{sc}} \rightarrow S_{\ell}$ le revêtement universel de $S_{\ell}\left(\operatorname{sur} \mathbb{Z}_{\ell}\right.$ ) et posons

$$
S_{\ell}(R)_{u} \text { l'image de } S_{\ell, \mathrm{sc}}(R) \text { dans } S_{\ell}(R) \text {, pour } R \in\left\{\mathbb{Z}_{\ell}, \mathbb{Q}_{\ell}, \mathbb{F}_{\ell}\right\} \text {. }
$$

Si $\ell \geq 5$ alors le groupe $S_{\ell}\left(\mathbb{F}_{\ell}\right)_{u}$ est le sous-groupe de $S_{\ell}\left(\mathbb{F}_{\ell}\right)$ engendré par les éléments unipotents. C'est également le groupe des commutateurs de $S_{\ell}\left(\mathbb{F}_{\ell}\right)$. Le point clef que nous utilisons peut s'énoncer ainsi.

Proposition 10.3 [Wintenberger 2002]. L'indice $S_{\ell}\left(\mathbb{Z}_{\ell}\right)_{u}$ dans $S_{\ell}\left(\mathbb{Z}_{\ell}\right)$ est borné indépendamment de $\ell$. Pour tout premier $\ell$ assez grand, le groupe $S_{\ell}\left(\mathbb{Z}_{\ell}\right)_{u}$ est contenu dans $G_{\ell}$. En particulier, on a les inclusions $S_{\ell}\left(\mathbb{Z}_{\ell}\right)_{u} \subset G_{\ell} \cap S_{\ell}\left(\mathbb{Z}_{\ell}\right) \subset S_{\ell}\left(\mathbb{Z}_{\ell}\right)$ avec indices finis, bornés indépendamment de $\ell$.

Cet énoncé découle de l'énoncé plus précis suivant.

Proposition 10.4 [Wintenberger 2002]. On a l'égalité $S_{\ell}\left(\mathbb{Z}_{\ell}\right)_{u}=S_{\ell}\left(\mathbb{Z}_{\ell}\right) \cap S_{\ell}\left(\mathbb{Q}_{\ell}\right)_{u}$. De plus, si le centre $Z\left(S_{\ell, \mathrm{sc}}\right)$ est de cardinal premier à $\ell$ alors $S_{\ell}\left(\mathbb{Z}_{\ell}\right)_{u}$ est l'image réciproque de $S_{\ell}\left(\mathbb{F}_{\ell}\right)_{\text {u }}$ par $\pi_{\ell}: S_{\ell}\left(\mathbb{Z}_{\ell}\right) \rightarrow S_{\ell}\left(\mathbb{F}_{\ell}\right)$, le morphisme de réduction modulo $\ell$. Pour tout premier $\ell$ assez grand, le groupe $S_{\ell}\left(\mathbb{Z}_{\ell}\right)_{u}$ est contenu dans $G_{\ell}$. Si $\ell$ est assez grand, alors l'indice $\left(S_{\ell}\left(\mathbb{Z}_{\ell}\right): S_{\ell}\left(\mathbb{Z}_{\ell}\right)_{u}\right)$ est majoré par c $(2 \operatorname{dim} A):=$ $\operatorname{ppcm}\{n \mid n \leq 2 \operatorname{dim} A\}$.

La démonstration du théorème 10.1 est maintenant immédiate à partir des propositions 10.2 et 10.3 . Comme $S$ (resp. $C$ ) est le groupe dérivé (resp. la composante neutre du centre) de $\operatorname{MT}(A)$ on a $\operatorname{MT}(A)=C \cdot S$ et on en tire aisément que $\left(\operatorname{MT}(A)\left(\mathbb{Z}_{\ell}\right): C\left(\mathbb{Z}_{\ell}\right) \cdot S\left(\mathbb{Z}_{\ell}\right)\right)$ est borné indépendamment de $\ell$. La proposition 10.2 fournit un sous-groupe $C_{1}$ de $C\left(\mathbb{Z}_{\ell}\right) \cap \rho_{\ell^{\infty}}\left(G_{K}\right)$ d'indice fini dans 
$C\left(\mathbb{Z}_{\ell}\right)$, tandis que la proposition 10.3 , jointe à l'hypothèse $S_{\mathbb{Q}_{\ell}}=S_{\ell}$ fournit un sous-groupe $S_{1}$ de $S\left(\mathbb{Z}_{\ell}\right) \cap \rho_{\ell \infty}\left(G_{K}\right)$ d'indice fini dans $S\left(\mathbb{Z}_{\ell}\right)$. On conclut bien alors que $\left(M T(A)\left(\mathbb{Z}_{\ell}\right): \rho\left(G_{K}\right)\right) \leq\left(M T(A)\left(\mathbb{Z}_{\ell}\right): C_{1} \cdot S_{1}\right)$ est borné indépendamment de $\ell$.

Donnons maintenant la description promise du centre du groupe de MumfordTate.

Notons $L=\prod_{i} L_{i}$ le centre de $\operatorname{End}^{0}(A)$; chaque $L_{i}$ est un corps de nombres et la décomposition correspond à la décomposition de $A$ à isogénie près en composantes isotypiques, i.e., $A \cong \prod_{i} A_{i}$ avec $A_{i}=B_{i}^{m_{i}}$ et $B_{i}$ absolument simple. On pose aussi $T_{L}=\prod_{i} \operatorname{Res}_{L_{i} / \mathbb{Q}}\left(\mathbb{G}_{m, L_{i}}\right)$ et on note $\operatorname{det}_{L_{i}}: \operatorname{Res}_{L_{i} / \mathbb{Q}}\left(\mathrm{GL}_{V_{i}, L_{i}}\right) \rightarrow \operatorname{Res}_{L_{i} / \mathbb{Q}}\left(\mathbb{G}_{m, L_{i}}\right)$ et $\operatorname{det}_{L}=\prod_{i} \operatorname{det}_{L_{i}}$. La restriction de $\operatorname{det}_{L}$ au tore $T_{L}$ donne une isogénie $\delta: T_{L} \rightarrow T_{L}$ qui peut être explicitée comme l'application $x=\left(x_{i}\right)_{i \in I} \mapsto\left(x_{i}^{d_{i}}\right)_{i \in I}$, où $d_{i}:=$ $2 \operatorname{dim} A_{i} /\left[L_{i}: \mathbb{Q}\right]$. Introduisons une extension auxiliaire $\tilde{L}$ finie, galoisienne sur $\mathbb{Q}$ et contenant les $L_{i}$; on définit ensuite la "norme" (cf. [Ichikawa 1991, p. 135]) :

$$
\begin{gathered}
\Psi_{i}: \operatorname{Res}_{\tilde{L} / \mathbb{Q}} \mathbb{G}_{m, \tilde{L}} \rightarrow \operatorname{Res}_{L_{i} / \mathbb{Q}} \mathbb{G}_{m, L_{i}}, \\
\Psi=\prod_{i} \Psi_{i}: \operatorname{Res}_{\tilde{L} / \mathbb{Q}} \mathbb{G}_{m, \tilde{L}} \rightarrow \prod_{i} \operatorname{Res}_{L_{i} / \mathbb{Q}} \mathbb{G}_{m, L_{i}} .
\end{gathered}
$$

On a alors une description de la composante neutre du centre du groupe de Mumford-Tate comme le sous-tore de $T_{L}$ vérifiant $\delta(C)=\operatorname{Im} \Psi$ (aux notations près, c'est la proposition 1.2.1 de [Ichikawa 1991]).

Proposition 10.5 (cf. [Ichikawa 1991; Serre 1986a]). Le tore $C=C_{A}$ est le soustore de $T_{L}$ tel que $\delta(C)=\Psi\left(T_{L}\right)$.

Le lien avec les représentations $\ell$-adiques peut être décrit ainsi (cf. [Serre 1986b]).

Chaque morceau $V_{\ell}\left(A_{i}\right)$ est libre de $\operatorname{rang} d_{i}$ sur $L_{i} \otimes \mathbb{Q}_{\ell} ;$ si l'on pose $L_{\ell}:=L \otimes \mathbb{Q}_{\ell}$,

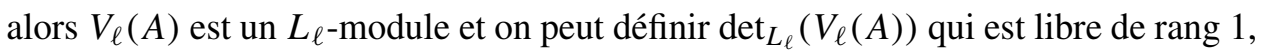
ce qui fournit une représentation $\ell$-adique abélienne à valeurs dans $T_{L}$ :

$$
\phi_{\ell}: G_{K} \rightarrow L_{\ell}^{\times}=\left(L \otimes \mathbb{Q}_{\ell}\right)^{\times}=T_{L}\left(\mathbb{Q}_{\ell}\right) .
$$

Par la théorie abélienne de Serre il existe un module $\mathfrak{m}$ et un homomorphisme de groupes algébriques associé $S_{\mathfrak{m}} \rightarrow T_{L}$ induisant la représentation de la manière suivante ; rappelons (cf. [Serre 1998]) que $S_{\mathfrak{m}}$ est un $\mathbb{Q}$-groupe algébrique extension du groupe fini $C_{\mathfrak{m}}$ des classes d'idèles modulo $\mathfrak{m}$ par un tore $T_{\mathfrak{m}}$. Si on note $\varepsilon_{\ell}: G_{K} \rightarrow S_{\mathfrak{m}}\left(\mathbb{Q}_{\ell}\right)$ la représentation définie dans [Serre 1998], alors $\phi_{\ell}=\phi \circ \varepsilon_{\ell}$.

Le lien avec le centre du groupe de Hodge est que $\Psi\left(T_{L}\right)$ est la composante neutre de $\phi\left(S_{\mathfrak{m}}\right)$. Ce fait appelé "exercice embêtant" dans [Serre 1986b] est équivalent à l'égalité de la composante connexe des centres de $G_{\ell}$ et $\mathrm{MT}(A)_{\mathbb{Q}_{\ell}}$ citée ci-dessus. 
Quelques cas de la conjecture de Mumford-Tate. Pour énoncer le premier résultat en vue, nous rappelons la notation suivante, cf. (1) :

$$
\Sigma=\left\{g \geq 1 \mid \exists k \geq 3, \text { impair, } \exists a \geq 1,2 g=(2 a)^{k} \text { ou } 2 g=\left(\begin{array}{c}
2 k \\
k
\end{array}\right)\right\} .
$$

Théorème 10.6. Soit $h$ un entier tel que $h \notin \Sigma$ et soit $A / K$ une variété abélienne de type II telle que le centre de $\operatorname{End}(A) \otimes \mathbb{Q}$ est réduit à $\mathbb{Q}$. On suppose que A est de dimension $g=2 h$. La conjecture de Mumford-Tate est vraie pour A et le groupe de Mumford-Tate associé à A est $\mathrm{GSp}_{2 h, \mathbb{Q}}$.

Démonstration. Il s'agit tout simplement d'appliquer la proposition 4.7 de Pink [1998]. Précisément : soit $\ell$ un nombre premier suffisamment grand; décomposons le module de Tate $V_{\ell}$ en somme de 2 copies isomorphes $W_{\ell}$ sur $\mathbb{Q}_{\ell}$. Les $W_{\ell}$ donnent des représentations de dimension $2 h$, de type Mumford-Tate fort (puisque c'est le cas par [Pink 1998, theorem 5.10] pour les $V_{\ell}$ ), fidèles, symplectiques, absolument irréductibles du groupe dérivé de $G_{\ell}$. On peut donc appliquer la proposition $4.7 \mathrm{de}$ Pink qui nous dit que si $h$ est en dehors de l'ensemble exceptionnel $\Sigma$ alors $G_{\ell}$ est isomorphe à $\mathrm{GSp}_{2 h, \mathbb{Q}}$.

Pour énoncer le résultat suivant, rappelons que si une variété abélienne $A$ a réduction semi-stable en une place $v$, la composante neutre de la fibre spéciale est une extension d'une variété abélienne $B_{0}$ par un tore que nous noterons $T_{0}$; la dimension de ce tore s'appelle la dimension torique. De plus, si ce tore est non trivial (i.e., s'il y a mauvaise réduction) l'anneau des endomorphismes de $A$ agit fidèlement sur ce tore. En conséquence, si $A$ est de type I et $e=\left[\operatorname{End}^{0}(A): \mathbb{Q}\right]$ (resp. de type II et $4 e=\left[\operatorname{End}^{0}(A): \mathbb{Q}\right]$ ) alors la dimension de $T_{0}$ est un multiple de $e$ (resp. de $2 e$ ).

Théorème 10.7. Soit A une variété abélienne simple de type I ou II, définie sur un corps de nombres $K$. On note e le degré sur $\mathbb{Q}$ du centre de $\operatorname{End}^{0}(A)$ et $d:=1$, si A est de type I, et $d:=2$ si A est de type II. Supposons de plus que:

(T) Il existe une place de $K$ où A possède réduction semi-stable de dimension torique de.

Alors A est pleinement de type Lefschetz.

Commençons par rappeler quelques résultats de Grothendieck décrits dans [SGA 7 I 1972]. On étudie la réduction modulo un idéal premier donc on peut passer à un anneau local que l'on peut d'ailleurs compléter et qu'on notera $\mathcal{O}$; dans le langage de [SGA $7_{\text {I }}$ 1972], le spectre de $\mathcal{O}$ est un trait. Tout schéma quasi-fini $X / \mathcal{O}$ se décompose en

$$
X=X^{\mathrm{f}} \sqcup X^{\prime},
$$

où $X^{\mathrm{f}}$ est un schéma fini sur $\mathcal{O}$ et $X^{\prime}$ est un schéma égal à sa fibre générique. On choisit un nombre premier $\ell$ assez grand pour qu'il ne divise pas le cardinal du 
groupe des composantes de la fibre spéciale du modèle de Néron sur $\mathcal{O}$ de $A$. En décomposant ainsi le schéma quasi-fini $A\left[\ell^{n}\right]$ et en prenant la limite on obtient la partie fixe $T_{\ell}(A)^{\mathrm{f}} \subset T_{\ell}(A)$. En considérant la partie torique de la fibre spéciale $A_{0}^{0}$ du modèle de Néron

$$
0 \rightarrow T_{0} \rightarrow A_{0}^{0} \rightarrow B_{0} \rightarrow 0
$$

on obtient la partie torique $T_{\ell}(A)^{\mathrm{t}} \subset T_{\ell}(A)^{\mathrm{f}}$, qui est de $\operatorname{rang} \mu:=\operatorname{dim} T_{0}$ (i.e., égal à la dimension torique). Le résultat clef de Grothendieck décrit ces sous-modules en termes de l'action du groupe d'inertie, noté $I$ et s'énonce ainsi (le premier point est la proposition 2.2.5 de [SGA 7 1 1972], le deuxième le théorème 2.4 de [SGA 7 1972]).

Théorème 10.8 [SGA 7 1 1972]. Avec les notations précédentes, on a :

(1) $T_{\ell}(A)^{\mathrm{f}}=T_{\ell}(A)^{I}$.

(2) Soit Ǎ la duale de A, désignons par $\perp$ l'orthogonal au sens de l'accouplement canonique de Weil $T_{\ell}(A) \times T_{\ell}(\check{A}) \rightarrow T_{\ell}\left(\mathbb{G}_{m}\right)$. Alors

$$
T_{\ell}(A)^{\mathrm{t}}=T_{\ell}(A)^{\mathrm{f}} \cap\left(T_{\ell}(\check{A})^{\mathrm{f}}\right)^{\perp}=T_{\ell}(A)^{I} \cap\left(T_{\ell}(\check{A})^{I}\right)^{\perp} .
$$

De plus, si on note $U=V_{\ell}(A)=T_{\ell}(A) \otimes_{\mathbb{Z}_{\ell}} \mathbb{Q}_{\ell}, U_{1}=V_{\ell}(A)^{\mathrm{f}}$ et $U_{2}=V_{\ell}(A)^{\mathrm{t}}$, et si l'on identifie $V_{\ell}(A)$ et $V_{\ell}(\check{A})$ via une polarisation fixée, on a donc $U_{2}=U_{1} \cap U_{1}^{\perp}$. L'inertie agit de plus trivialement sur $U_{2}$ et sur $U / U_{2}$ (mais non trivialement sur $U$ si l'on suppose qu'il y a mauvaise réduction).

Démonstration du théorème 10.7. Notons comme précédemment $E$ le centre de $\operatorname{End}^{0}(A)$. Il suffit de montrer que, pour un $\ell$, le groupe $H_{\ell}$ est le produit des $\mathrm{Sp}_{2 h, E_{\lambda}}$, quand $\lambda$ parcourt les idéaux premiers de $E$ au dessus de $\ell$. On choisit un $\ell$ assez grand et totalement décomposé dans $E / \mathbb{Q}$; d'après le lemme de relèvement (lemme 2.6), il suffit de voir que l'image de Galois modulo $\ell$ contient le produit des $\mathrm{Sp}_{2 h, \mathbb{F}_{\ell}}$. Chris Hall [2011] montre dans le cas où $\operatorname{End}(A)=\mathbb{Z}$ (i.e., $e=d=1$ ) que l'hypothèse $(\mathrm{T})$ entraîne ceci. Nous expliquons comment adapter ses arguments au cas plus général.

Considérons les sous-modules décrits ci-dessus $U_{2} \subset U_{1} \subset V_{\ell}(A)$ et rappelons que $\mu=\operatorname{rang} U_{2}$ est la dimension torique. L'inertie opère non trivialement sur $V_{\ell}(A)$ puisqu'il y a mauvaise réduction mais trivialement sur $U_{2}$ et $V_{\ell}(A) / U_{2}$. Si $g_{I}$ désigne un générateur topologique du quotient maximal pro- $\ell$-fini de $I$, sa matrice $\rho_{\ell}\left(g_{I}\right)$ est donc conjuguée à une matrice $\left(\begin{array}{cc}I_{\mu} & B \\ 0 & I_{2 g-\mu}\end{array}\right)$.

On peut "découper" les $U_{i}$, qui sont des $\mathbb{Q}_{\ell}$-espaces vectoriels, en introduisant $e_{\lambda}$ l'idempotent de $E_{\ell}=E \otimes_{\mathbb{Q}} \mathbb{Q}_{\ell}$ qui projette $E_{\ell}$ sur $E_{\lambda}$ et en notant $U_{i, \lambda}$ l'image $e_{\lambda} U_{i}$. On obtient alors la décomposition cherchée.

Lemme 10.9. Les sous-modules galoisiens $U_{1}$ "partie fixe" et $U_{2}$ "partie torique" sont stables par $\operatorname{End}(A)$ et se décomposent ainsi:

(1) (Type I) Les $U_{i}$ se décomposent en $U_{i}=\prod_{\lambda} U_{i, \lambda}$. 
(2) (Type II) Les $U_{i}$ se décomposent en $U_{i}=\prod_{\lambda}\left(U_{i, \lambda} \oplus U_{i, \lambda}\right)$.

Démonstration (du lemme). L'action des endomorphismes commute avec celle du groupe de Galois et en particulier du groupe d'inertie, ce qui entraîne la première affirmation. De plus, pour tout endomorphisme $\alpha$, on a

$$
<\alpha v, v^{\prime}>=<v, \alpha^{\dagger} v^{\prime}>
$$

où $<\cdot, \cdot>$ désigne l'accouplement de Weil et $\dagger$ l'involution de Rosati associée à la polarisation choisie; ainsi les décompositions de la représentation galoisienne décrites dans la proposition 3.5 induisent celles sur $U_{1}$ et $U_{2}$.

L'hypothèse ( $\mathrm{T}$ ) se traduit en disant que $d e=\operatorname{rang} U_{2}=d \sum_{\lambda} \operatorname{rang} U_{i, \lambda}$, ce qui impose rang $U_{i, \lambda}=1$. Si l'on écrit maintenant la matrice de $\rho\left(g_{I}\right)$ par blocs, on voit que chaque bloc est une transvection. Les arguments de [Hall 2011] s'appliquent alors, permettant de montrer que chaque bloc de la représentation modulo $\ell$ contient dans son image $\operatorname{Sp}_{2 h}\left(\mathbb{F}_{\ell}\right)$, ce qui achève la preuve du théorème 10.7.

Théorème 10.10. Soit $s \geq 1$ un entier et soient $A_{1}, \ldots, A_{s}$ des variétés abéliennes, deux à deux non isogènes, chaque $A_{i}$ étant pleinement de type Lefschetz, de type I ou II, de dimension relative un entier $h_{i}$. On a dans ce cas

$$
\begin{gathered}
\operatorname{Hdg}\left(\prod_{i=1}^{s} A_{i}\right)=\prod_{i=1}^{s} \operatorname{Hdg}\left(A_{i}\right)=\prod_{i=1}^{s} \operatorname{Res}_{E_{i} / \mathbb{Q}} \operatorname{Sp}_{2 h_{i}, E_{i}}, \\
\text { pour tout } \ell, \quad H_{\ell}\left(\prod_{i=1}^{s} A_{i}\right)=\prod_{i=1}^{s} H_{\ell}\left(A_{i}\right)=\prod_{i=1}^{s} \prod_{\lambda_{i} \mid \ell} \operatorname{Sp}_{2 h_{i}, E_{\lambda_{i}}},
\end{gathered}
$$

où $\lambda_{i}$ parcourt les places de $E_{i}$ au dessus de $\ell$.

Démonstration. Nous expliquons la preuve dans le cadre $\ell$-adique qui s'appuie sur l'article de Lombardo [2016] (le cas complexe est plus simple et s'appuie de manière parallèle sur l'article antérieur d'Ichikawa [1991]). Il s'agit en fait d'appliquer le théorème 4.1 de [Lombardo 2016]. Pour cela il nous suffit de vérifier que les hypothèses de ce théorème sont satisfaites. Nos hypothèses entraînent que pour tout entier $i$, on a $\operatorname{Hdg}\left(A_{i}\right) \times \mathbb{C}_{\ell}=\operatorname{Sp}_{2 h_{i}, \mathbb{C}_{\ell}}^{\left[E_{i}: \mathbb{Q}\right]}$, où $E_{i}$ est le centre de $\operatorname{End}\left(A_{i}\right) \otimes \mathbb{Q}$. Or les automorphismes de (l'algèbre de Lie de) $\mathrm{Sp}_{2 h_{i}, \mathbb{C}_{\ell}}$ sont intérieurs et les automorphismes intérieurs préservent les plus haut poids (cf. remarque 3.8 de [Lombardo 2016]), donc le point (3) des hypothèses du théorème 4.1 de [Lombardo 2016] est vérifié. Les points (1) et (2) sont immédiats dans notre situation, d'où la conclusion.

Corollaire 10.11. Soit $s \geq 1$ un entier et soient $A_{1}, \ldots, A_{s}$ des variétés abéliennes, deux à deux non isogènes, chaque $A_{i}$ étant de type I ou II, de dimension relative un entier $h_{i}$. Pour chaque $i$, on suppose que l'une des hypothèses suivantes est vérifiée: 
(1) Le centre de $\operatorname{End}(A) \otimes \mathbb{Q}$ est réduit à $\mathbb{Q}$ et l'entier $h_{i}$ n'est pas dans l'ensemble exceptionnel $\Sigma$.

(2) L'entier $h_{i}$ est égal à deux ou est impair.

(3) La variété abélienne $A_{i}$ est de type I (resp. II) et possède une place de mauvaise réduction semi-stable avec dimension torique $e_{i}$ (resp. avec dimension torique $2 e_{i}$ ).

Sous ces hypothèses on a alors

$$
\begin{gathered}
\operatorname{Hdg}\left(\prod_{i=1}^{s} A_{i}\right)=\prod_{i=1}^{s} \operatorname{Hdg}\left(A_{i}\right)=\prod_{i=1}^{s} \operatorname{Res}_{E_{i} / \mathbb{Q}} \operatorname{Sp}_{2 h_{i}, E_{i}}, \\
\text { pour tout } \ell, \quad H_{\ell}\left(\prod_{i=1}^{s} A_{i}\right)=\prod_{i=1}^{s} H_{\ell}\left(A_{i}\right)=\prod_{i=1}^{s} \prod_{\lambda_{i} \mid \ell} \operatorname{Sp}_{2 h_{i}, E_{\lambda_{i}}},
\end{gathered}
$$

où $\lambda_{i}$ parcourt les places de $E_{i}$ au dessus de $\ell$.

Démonstration. Soit $i$ un entier dans l'ensemble $\{1, \ldots, s\}$. Si l'hypothèse (1) est vérifiée alors par le théorème 5.14 de [Pink 1998] (dans le cas de type I) ou par le théorème 10.6 ci-dessus (dans le cas de type II) on en déduit que $A$ est pleinement de type Lefschetz, de groupe de Hodge, $\mathrm{Sp}_{2 h_{i}}$. Si l'hypothèse (2) est vérifiée, là encore la même conclusion vaut en appliquant cette fois le théorème $\mathrm{A}$ de [Banaszak et al. 2006] (cf. [Lombardo 2016, remarque 2.25] pour le cas de dimension relative 2). Il suffit donc d'appliquer le théorème 10.10 ci-dessus pour conclure.

Remarque 10.12. On peut bien sûr énoncer le théorème et le corollaire précédents en remplaçant $\prod_{i=1}^{s} A_{i}$ par $\prod_{i=1}^{s} A_{i}^{m_{i}}$ puisque $\operatorname{Hdg}\left(\prod_{i=1}^{s} A_{i}^{m_{i}}\right)=\operatorname{Hdg}\left(\prod_{i=1}^{s} A_{i}\right)$ et $H_{\ell}\left(\prod_{i=1}^{s} A_{i}^{m_{i}}\right)=H_{\ell}\left(\prod_{i=1}^{s} A_{i}\right)$.

\section{Remerciements}

Les auteurs remercient le rapporteur, dont la lecture attentive et les corrections et suggestions judicieuses ont permis d'améliorer la présentation de ce texte. Une partie de ces recherches a bénéficié du soutien de l'ANR "HAMOT".

\section{Bibliographie}

[Banaszak et al. 2006] G. Banaszak, W. Gajda et P. Krasoń, "On the image of $l$-adic Galois representations for abelian varieties of type I and II", Doc. Math. Extra Vol. (2006), 35-75. MR 2290584

[Borovor̆ 1974] M. V. Borovoŭ, “О действии группы Галуа на рациональные классы когомологий ( $p, p)$ абелевых многообразий”, Mat. Sb. (N.S.) 94 (136):4 (1974), 649-652. Traduction anglaise : "On the Galois action on rational cohomology classes of type $(p, p)$ of abelian varieties,", Math. USSR Sb. 23 :4, 613-616. MR 0352101

[Breuer 2010] F. Breuer, "Torsion bounds for elliptic curves and Drinfeld modules", J. Number Theory 130:5 (2010), 1241-1250. MR 2607312 Zbl 1227.11072 
[Chi 1990] W. C. Chi, "On the Tate modules of absolutely simple abelian varieties of type II", Bull. Inst. Math. Acad. Sinica 18:2 (1990), 85-95. MR 1071226 Zbl 0729.14033

[Chi 1992] W. C. Chi, " $l$-adic and $\lambda$-adic representations associated to abelian varieties defined over number fields”, Amer. J. Math. 114:2 (1992), 315-353. MR 1156568 Zbl 0795.14024

[Deligne et al. 1982] P. Deligne, J. S. Milne, A. Ogus et K.-y. Shih, Hodge cycles, motives, and Shimura varieties, Lecture Notes in Math. 900, Springer, Berlin, 1982. MR 654325 Zbl 0465.00010

[Hall 2011] C. Hall, "An open-image theorem for a general class of abelian varieties", Bull. Lond. Math. Soc. 43:4 (2011), 703-711. MR 2820155 Zbl 1225.11083

[Hindry et Ratazzi 2010] M. Hindry et N. Ratazzi, "Torsion dans un produit de courbes elliptiques", J. Ramanujan Math. Soc. 25:1 (2010), 81-111. MR 2643390 Zbl 1206.11075

[Hindry et Ratazzi 2012] M. Hindry et N. Ratazzi, "Points de torsion sur les variétés abéliennes de type GSp”, J. Inst. Math. Jussieu 11:1 (2012), 27-65. MR 2862374 Zbl 1311.11050

[Ichikawa 1991] T. Ichikawa, "Algebraic groups associated with abelian varieties", Math. Ann. 289:1 (1991), 133-142. MR 1087242 Zbl 0697.14031

[Larsen et Pink 1995] M. Larsen et R. Pink, "Abelian varieties, $l$-adic representations, and $l$ independence”, Math. Ann. 302:3 (1995), 561-579. MR 1339927 Zbl 0867.14019

[Lombardo 2016] D. Lombardo, "On the $\ell$-adic Galois representations attached to nonsimple Abelian varieties”, Ann. Inst. Fourier (Grenoble) 66:3 (2016), 1217-1245. MR 3494170

[Masser 1986] D. Masser, lettre à Daniel Bertrand, 10 novembre 1986.

[Milne 1999] J. S. Milne, "Lefschetz classes on abelian varieties", Duke Math. J. 96:3 (1999), 639-675. MR 1671217 Zbl 0976.14009

[Moonen 1999] B. Moonen, "Notes on Mumford-Tate groups", lecture notes, 1999, Voir http:// www.math.ru.nl/ bmoonen/Lecturenotes/CEBnotesMT.pdf.

[Murty 1984] V. K. Murty, "Exceptional Hodge classes on certain abelian varieties", Math. Ann. 268:2 (1984), 197-206. MR 744607 Zbl 0521.14004

[Noot 1995] R. Noot, "Abelian varieties-Galois representation and properties of ordinary reduction", Compositio Math. 97:1-2 (1995), 161-171. MR 1355123 Zbl 0868.14021

[Piatetski-Shapiro 1971] I. I. Piatetski-Shapiro, “Взаимоотношения между гипотезами Тайта и Ходжа для абелевых многообразий”, Mat. Sb. (N.S.) 85 (127) (1971), 610-620. Traduction anglaise : "Interrelations between the Tate and Hodge conjectures for abelian varieties", Math. USSR Sb. 14 :4, 615-624. MR 0294352

[Pink 1998] R. Pink, " $l$-adic algebraic monodromy groups, cocharacters, and the Mumford-Tate conjecture”, J. Reine Angew. Math. 495 (1998), 187-237. MR 1603865

[Ratazzi 2007] N. Ratazzi, "Borne sur la torsion dans les variétés abéliennes de type CM", Ann. Sci. École Norm. Sup. (4) 40:6 (2007), 951-983. MR 2419854 Zbl 1140.14041

[Ribet 1976] K. A. Ribet, "Galois action on division points of Abelian varieties with real multiplications”, Amer. J. Math. 98:3 (1976), 751-804. MR 0457455 Zbl 0348.14022

[Serre 1986a] J.-P. Serre, lettre à Ken Ribet, 7 mars 1986. §138 dans ses CEuvres, IV, 1985-1998, Springer, Berlin, 2000.

[Serre 1986b] J.-P. Serre, lettre à Marie-France Vignéras, 10 février 1986. $\$ 137$ dans ses CEuvres, IV, 1985-1998, Springer, Berlin, 2000. 
[Serre 1986c] J.-P. Serre, "Résumé des cours au Collège de France de 1985-1986”, 1986. §136 dans ses Euvres, IV : 1985-1998, Springer, Berlin, 2000.

[Serre 1998] J.-P. Serre, Abelian l-adic representations and elliptic curves, Research Notes in Mathematics 7, A K Peters, Wellesley, MA, 1998. Revised reprint of the 1968 original. MR 1484415 Zbl 0902.14016

[Serre 2013] J.-P. Serre, "Un critère d'indépendance pour une famille de représentations $\ell$-adiques", Comment. Math. Helv. 88:3 (2013), 541-554. MR 3093502 Zbl 1317.14040

[SGA 7 1972] A. Grothendieck, Groupes de monodromie en géométrie algébrique, I: Exposés I-II, VI-IX (Séminaire de Géométrie Algébrique du Bois Marie 1967-1969), Lecture Notes in Math. 288, Springer, Berlin, 1972. MR 0354656 Zbl 0237.00013

[Ullmo et Yafaev 2013] E. Ullmo et A. Yafaev, "Mumford-Tate and generalised Shafarevich conjectures”, Ann. Math. Qué. 37:2 (2013), 255-284. MR 3117743 Zbl 06350059

[Vasiu 2008] A. Vasiu, "Some cases of the Mumford-Tate conjecture and Shimura varieties", Indiana Univ. Math. J. 57:1 (2008), 1-75. MR 2400251 Zbl 1173.11039

[Wintenberger 2002] J.-P. Wintenberger, "Démonstration d'une conjecture de Lang dans des cas particuliers", J. Reine Angew. Math. 553 (2002), 1-16. MR 1944805 Zbl 1048.14008

Communicated by Joseph H. Silverman

Received 2015-05-25 Revised 2016-07-14 Accepted 2016-08-12

marc.hindry@imj-prg.fr Institut de Mathématiques Jussieu - Paris Rive Gauche, Université Paris Diderot, Bâtiment Sophie Germain, 8 place Aurélie Nemours, 75013 Paris, France

nicolas.ratazzi@math.u-psud.fr Equipe d'Arithmétique et Géométrie Algébrique, Université Paris-Sud, Bâtiment 425, 91405 Orsay, France 


\section{Algebra \& Number Theory}

msp.org/ant

\section{EDITORS}

MANAGING EDITOR

Bjorn Poonen

Massachusetts Institute of Technology

Cambridge, USA

\author{
EDITORIAL BOARD CHAIR \\ David Eisenbud \\ University of California \\ Berkeley, USA
}

BOARD OF EDITORS

$\begin{aligned} \text { Dave Benson } & \text { University of Aberdeen, Scotland } & \text { Susan Montgomery } & \text { University of Southern California, USA } \\ \text { Richard E. Borcherds } & \text { University of California, Berkeley, USA } & \text { Shigefumi Mori } & \text { RIMS, Kyoto University, Japan } \\ \text { John H. Coates } & \text { University of Cambridge, UK } & \text { Raman Parimala } & \text { Emory University, USA } \\ \text { J-L. Colliot-Thélène } & \text { CNRS, Université Paris-Sud, France } & \text { Jonathan Pila } & \text { University of Oxford, UK } \\ \text { Brian D. Conrad } & \text { Stanford University, USA } & \text { Anand Pillay } & \text { University of Notre Dame, USA } \\ \text { Hélène Esnault } & \text { Freie Universität Berlin, Germany } & \text { Victor Reiner } & \text { University of Minnesota, USA } \\ \text { Hubert Flenner } & \text { Ruhr-Universität, Germany } & \text { Peter Sarnak } & \text { Princeton University, USA } \\ \text { Sergey Fomin } & \text { University of Michigan, USA } & \text { Joseph H. Silverman } & \text { Brown University, USA } \\ \text { Edward Frenkel } & \text { University of California, Berkeley, USA } & \text { Michael Singer } & \text { North Carolina State University, USA } \\ \text { Andrew Granville } & \text { Université de Montréal, Canada } & \text { Vasudevan Srinivas } & \text { Tata Inst. of Fund. Research, India } \\ \text { Joseph Gubeladze } & \text { San Francisco State University, USA } & \text { J. Toby Stafford } & \text { University of Michigan, USA } \\ \text { Roger Heath-Brown } & \text { Oxford University, UK } & \text { Ravi Vakil } & \text { Stanford University, USA } \\ \text { Craig Huneke } & \text { University of Virginia, USA } & \text { Michel van den Bergh } & \text { Hasselt University, Belgium } \\ \text { Kiran S. Kedlaya } & \text { Univ. of California, San Diego, USA } & \text { Marie-France Vignéras } & \text { Université Paris VII, France } \\ \text { János Kollár } & \text { Princeton University, USA } & \text { Kei-Ichi Watanabe } & \text { Nihon University, Japan } \\ \text { Yuri Manin } & \text { Northwestern University, USA } & \text { Efim Zelmanov } & \text { University of California, San Diego, USA } \\ \text { Philippe Michel } & \text { École Polytechnique Fédérale de Lausanne } & \text { Shou-Wu Zhang } & \text { Princeton University, USA }\end{aligned}$

PRODUCTION

production@msp.org

Silvio Levy, Scientific Editor

See inside back cover or msp.org/ant for submission instructions.

The subscription price for 2016 is US $\$ 290$ /year for the electronic version, and $\$ 485 /$ year (+\$55, if shipping outside the US) for print and electronic. Subscriptions, requests for back issues and changes of subscribers address should be sent to MSP.

Algebra \& Number Theory (ISSN 1944-7833 electronic, 1937-0652 printed) at Mathematical Sciences Publishers, 798 Evans Hall \#3840, c/o University of California, Berkeley, CA 94720-3840 is published continuously online. Periodical rate postage paid at Berkeley, CA 94704, and additional mailing offices.

ANT peer review and production are managed by EditFLow ${ }^{\circledR}$ from MSP.

\section{PUBLISHED BY}

- mathematical sciences publishers

nonprofit scientific publishing

http://msp.org/

() 2016 Mathematical Sciences Publishers 


\section{Algebra \& Number Theory}

Volume $10 \quad$ No. $9 \quad 2016$

Torsion pour les variétés abéliennes de type I et II

MARC HINDRY and NICOLAS RATAZZI

Galois-generic points on Shimura varieties

ANNA CADORET and ARNO KRET

Extremality of loci of hyperelliptic curves with marked Weierstrass points

DAWEI CHEN and NiCOLA TARASCA

$\bar{\Re}_{15}$ is of general type

GREGOR BRUNS

A vanishing theorem for weight-one syzygies

LAWRENCE EIN, ROBERT LAZARSFELD and DAVID YANG

1983

Effective cones of cycles on blowups of projective space

IZZET COSKUN, JOHN LESIEUTRE and JOHN CHRISTIAN OTTEM

Cluster algebras and category $\mathrm{O}$ for representations of Borel subalgebras of quantum affine algebras

DAVID HERNANDEZ and BERNARD LECLERC 